\title{
Analytical solutions for periodic motions to chaos in nonlinear systems with/without time-delay
}

\author{
Albert C. J. Luo \\ Received: 7 June 2013 / Revised: 25 July 2013 / Accepted: 30 July 2013 / Published online: 30 September 2013 \\ (C) Springer-Verlag Berlin Heidelberg 2013
}

\begin{abstract}
In this paper, the analytical dynamics of periodic flows to chaos in nonlinear dynamical systems is presented from the ideas of Luo (Continuous dynamical systems, Higher Education Press/L\&H Scientific, Beijing/Glen Carbon, 2012). The analytical solutions of periodic flows and chaos in autonomous systems are discussed through the generalized harmonic balance method, and the analytical dynamics of periodically forced nonlinear dynamical systems is presented as well. The analytical solutions of periodic motions in free and periodically forced vibration systems are presented. The similar ideas are extended to time-delayed nonlinear systems. The analytical solutions of periodic flows to chaos for time-delayed, nonlinear systems with/without periodic excitations are presented, and time-delayed, nonlinear vibration systems will be also discussed. The analytical solutions of periodic flows and chaos are independent of small parameters, which are different from the traditional perturbation methods. The methodology presented herein will provide the accurate analytical solutions of periodic motions to chaos in dynamical systems with/without time-delay. This approach can handle nonlinear dynamical systems with either single time-delay or multiple time-delays.
\end{abstract}

Keywords Nonlinear systems - Time-delayed nonlinear systems $\cdot$ Periodic flows $\cdot$ Chaos $\cdot$ Analytical solutions

\section{Introduction}

Since the 18th century, one has been interested in periodic motions in dynamical systems. The Fourier series theory tells

A. C. J. Luo $(\varangle)$

Department of Mechanical and Industrial Engineering, Southern Illinois University Edwardsville, Edwardsville, IL 62026-1805, USA

e-mail: aluo@siue.edu that any periodic function can be expressed by a Fourier series of harmonic functions. In 1788, Lagrange [1] used the method of averaging for motions of moon, earth and sun in the three-body problem. In the end of 19th century, Poincare [2] provided the qualitative analysis of dynamical systems to determine periodic solutions and stability, and developed the perturbation theory for periodic solution. In addition, discovered was that the motion of a nonlinear coupled oscillator is sensitive to the initial condition and the motion in the vicinity of unstable fixed points of nonlinear oscillation systems may be stochastic under regular applied forces. In the 20th century, one followed the Poincare's ideas to develop and apply the qualitative theory to investigate the complexity of motions in dynamical systems. With the Poincare's influence, Birkhoff [3] continued the Poincare's work, and a proof of Poincare's geometric theorem was given. In 1927, Birkhoff [4] showed that both stable and unstable fixed points of nonlinear oscillation systems with two degrees of freedom must exist whenever their frequency ratio (or called resonance) is rational. The sub-resonances in periodic motions of such systems change the topological structures of phase trajectories, and the island chains are obtained when the dynamical systems can be renormalized with fine scales. In such qualitative and quantitative analysis, the Taylor series expansion and the perturbation analysis play an important role. However, the Taylor series expansion analysis is valid in the small finite domain under certain convergent conditions, and the perturbation analysis based on the small parameters, as an approximate estimate, is only acceptable for a very small domain with a short time period.

In 1920, van der Pol [5] used the averaging method to determine the periodic motions of self-excited systems in circuits, and the presence of natural entrainment frequencies in such a system was observed (also see [6]). In 1945, Cartwright and Littlewood [7] discussed the periodic motions 
of the van der Pol equation and proved the existence of periodic motions. In 1947, Cartwright and Littlewood [8] discussed the periodic motions of a generalized nonlinear equation based on the alike Duffing equation. In 1948, Levinson [9] used a piecewise linear model to describe the van der Pol equation and determined the existence of periodic motions. In 1949, Levinson [10] further developed the structures of periodic solutions in such a second order differential equation through the piecewise linear model, and discovered that infinite periodic solutions exist in such a piecewise linear model. From the Levinson's results, in 1967, Smale [11] used the topological point of view to present the Smale horseshoe with discontinuous mappings to describe the existence of infinite periodic motions. Further, a differentiable dynamical system theory was developed. Such a theory has been extensively used to interpret the homoclinic tangle phenomenon in nonlinear dynamical systems. Smale found the infinite many periodic motions, and a perfect minimal Cantor set near a homoclinic motion can be formed. In 1962, Melnikov [12] used the concept of Poincare [2] to investigate the behavior of trajectories of perturbed systems near autonomous Hamiltonian systems. In 1963, Melnikov [13] further investigated the behavior of trajectories of perturbed Hamiltonian systems, and the width of the separatrix splitting were approximately estimated. The width gives the domain of the chaotic motion in the vicinity of the generic separatrix. Even if the width of the separatix splitting was approximately estimated, the dynamics of the separatrix splitting was not developed.

Since nonlinear phenomena were observed in engineering, in 1918, Duffing [14] used the hardening spring model to investigate the vibration of electro-magnetized vibrating beam, and after then, the Duffing oscillator has been extensively used in structural dynamics. In addition to determining the existence of periodic motions in nonlinear differential equations of the second order in mathematics, one has applied the Poincare perturbation methods for periodic motions in nonlinear dynamical systems. In 1928, Fatou [15] provided the first proof of asymptotic validity of the method of averaging through the existence of solutions of differential equations. In 1935, Krylov and Bogolyubov [16] developed systematically the method of averaging (also see [17]). The classic perturbation methods for nonlinear oscillators were presented (e.g., [18-20]). In 1964, Hayashi [20] used the method of averaging and harmonic balance method to discuss the approximate periodic solutions of nonlinear systems and the corresponding stability. In 1973, Nayfeh [21] employed the multiple-scale perturbation method to develop approximate solutions of periodic motions in the Duffing oscillators. In 1976, Holmes and Rand [22] discussed the stability and bifurcation of periodic motions in the Duffing oscillator. In 1979, Nayfeh and Mook [23] applied the perturbation analysis to nonlinear structural vibrations via the Duffing oscillators, and Holmes [24] demonstrated chaotic motions in nonlinear oscillators through the Duffing oscillator with a twin-well potential. In 1980, Ueda [25] numerically simulated chaos via period-doubling of periodic motions of Duffing oscillators.

Based on the work of Melnikov [13], in 1981, Greenspan [26] extended the similar ideas to dissipative dynamical systems (also see, [27,28]). Further, the Melnikov method was developed for the global transversality in dissipative nonlinear systems. Once the global transversality to the separatrix exists, one thought that the Smale horseshoe presented in Smale [11] may exist, and furthermore chaos in such a nonlinear dynamical system may occur. However, from such a prediction based on the Melnikov method, one cannot observe the global transversality in nonlinear dynamical systems. The Smale horseshoe theory may not be adequate for continuous nonlinear dynamical systems rather than topological structures in discrete nonlinear systems. From the perturbation analysis, the Melnikov function was obtained for Hamiltonian systems with a small perturbation. One used such a function to analytically predict global behaviors (e.g., chaos) in the Hamiltonian systems with a small perturbation. Because of the perturbation analysis, the Melnikov method can give a reasonable analysis of the global behavior only when the perturbation is very small and close to zero. However, the perturbation in one-degree-of-freedom nonlinear systems is very small to zero, chaos in such nonlinear dynamical systems may not occur. So the Melnikov method may not help us understand the global behaviors of nonlinear dynamical systems. In 1995, Luo [29] used the Chirikov criterion to determine Hamiltonian chaos and applied the Melnikov function to investigate the global transversality (also see, [30]). The conclusion is the Melnikov method cannot provide an adequate prediction of chaotic motions in dissipative systems. For a better understanding of the Melnikov method, the results in Melnikov [13] should be revisited. Melnikov [13] presented a perturbation analysis to estimate the width of the separatrix splitting. Indeed, the width of the separatrix can be approximately estimated, but it cannot be used for predicting the existence of chaos. The Melnikov function is an approximate energy increment during a certain time period, which can be found in references (e.g., [31-33]). If the Melnikov function is zero, from a physical point of view, the system energy is conserved during a certain time period. Such a zero value of the Melnikov function does not imply that the flow has any global transversality to the separatrix. One has difficulty to find a connection from periodic motions to chaos. Thus, one continues using the perturbation analysis to determine the approximate analytical solution of periodic motions. In 1990, Coppola and Rand [34] determined limit cycles of nonlinear oscillators through elliptic functions in the method of averaging. In 1992, Wang et al. [35] used the harmonic balance method and the Floquet theory to investigate the nonlinear behaviors of the Duffing oscillator 
with a bounded potential well (also see, [36]). In 1997, Luo and Han [37] determined the stability and bifurcation conditions of periodic motions of the Duffing oscillator. However, only symmetric periodic motions of the Duffing oscillators were investigated. In 1999, Luo and Han [29] investigated the analytical prediction of chaos in nonlinear rods through the Duffing oscillator. In 2008, Peng et al. [38] presented the approximate symmetric solution of period-1 motions in the Duffing oscillator by the harmonic balance method with three harmonic terms. In 2012, Luo [39] developed a generalized harmonic balance method to get the approximate analytical solutions of periodic motions and chaos in nonlinear dynamical systems. This method used the finite Fourier series to express periodic motions and the coefficients are slowly time-varying. With averaging, a dynamical system of coefficients are obtained from which the steady-state solution are achieved and the corresponding stability and bifurcation are completed. Luo and Huang [40] used the generalized harmonic balance method with finite terms to obtain the analytical solution of period-1 motion of the Duffing oscillator with a twin-well potential. Luo and Huang [41] also employed a generalized harmonic balance method to find analytical solutions of period- $m$ motions in such a Duffing oscillator. The analytical bifurcation trees of periodic motions in the Duffing oscillator to chaos were obtained (also see, [42-47]). Such analytical bifurcation trees show the connection from periodic solutions to chaos analytically. To better understand nonlinear behaviors in nonlinear dynamical systems, the analytical solutions for the bifurcation trees of period-1 motions to chaos in a periodically forced oscillator with quadratic nonlinearity were presented in Luo and $\mathrm{Yu}$ [48-50], and period-m motions in the periodically forced, van der Pol equation was presented in Luo and Laken [51]. The analytical solutions for the van der Pol oscillator can be used to verify the conclusions in Cartwright and Littlewood [7] and Levinson [10]. The results for the quadratic nonlinear oscillator in Luo and Yu [48-50] analytically show the complicated period-1 motions and the corresponding bifurcation structures.

In recent years, time-delayed systems are of great interest since such systems extensively exist in engineering (e.g., $[52,53])$. The infinite dimensional state space causes the significant difficulty to understand such time-delayed problems. One tried to work on numerical methods to get the corresponding complicated behaviors. On the other hand, one is interested in the stability and bifurcation of equilibriums of the time-delayed systems (e.g., [54-56]). In addition, one is interested in periodic solutions in time-delayed dynamical systems. Perturbation methods have been used for such periodic motions in delayed dynamical systems. For instance, the approximate solutions of the time-delayed nonlinear oscillator were investigated by the method of multiple scales (e.g., $[57,58])$. The harmonic balance method was also used to determine approximate periodic solutions for delayed nonlinear oscillators (e.g., [59-61]). However, such approximate solutions of periodic motions in the time-delayed oscillators are based on one or two harmonic terms, which are not accurate enough. In addition, the corresponding stability and bifurcation analysis of such approximate solutions may not be adequate. In this paper, an alternative way will be presented for how to find the accurate analytical solutions of periodic flows in time-delayed dynamical systems. This method is without any small-parameter requirement. In addition, this approach can also be applicable to the coefficient varying with time.

In this paper, from Luo [39], the methods for the analytical periodic flows and chaos in nonlinear dynamical systems will be reviewed. The analytical solutions of periodic flows and chaos in autonomous systems will be presented first, and the analytical dynamics of periodically forced nonlinear dynamical systems will be discussed. For application purpose, the analytical solutions of periodic motions in free and periodically forced vibration systems will be presented. To extend the ideas to the time-delayed dynamical systems, herein, the analytical methods for the analytical solutions of periodic flows for time-delayed, nonlinear systems will be developed with/without periodic excitations, and time-delayed vibration systems will be also discussed.

\section{Dynamical systems without time-delay}

In this section, analytical periodic flows in autonomous and periodically forced, nonlinear dynamical systems will be discussed. A generalized harmonic balance method with the Fourier series expressions is presented for such analytical, periodic flows and chaos in nonlinear dynamical systems. The local stability and bifurcation theory of equilibriums in nonlinear autonomous systems of coefficients in the Fourier series solutions will be employed to classify analytical solutions of periodic flows and chaos in nonlinear dynamical systems.

\subsection{Autonomous nonlinear systems}

Periodic flows in autonomous dynamical systems will be presented first. If an autonomous nonlinear system has a periodic flow with period $T=2 \pi / \Omega$, then such periodic flow can be expressed by the Fourier series. The method is stated through the following theorem.

\section{Theorem 1 Consider a nonlinear dynamical system as}

$\dot{\mathbf{x}}=\mathbf{f}(\mathbf{x}, \mathbf{p}) \in \mathbf{R}^{n}$

where $\mathbf{f}(\mathbf{x}, \mathbf{p})$ is a $C^{r}$-continuous nonlinear function vector $(r \geq 1)$. If such a dynamical system has a periodic flow $\mathbf{x}^{*}(t)$ 
with finite norm $\left\|\mathbf{x}^{*}\right\|$ and $T=2 \pi / \Omega$, there is an analytical expression for the periodic flow of Eq. (1) in the form of

$\mathbf{x}^{*}(t)=\mathbf{a}_{0}(t)+\sum_{k=1}^{N} \mathbf{b}_{k}(t) \cos (k \Omega t)+\mathbf{c}_{k}(t) \sin (k \Omega t)$

with

$\mathbf{a}_{0}=\left(a_{01}, a_{02}, \ldots, a_{0 n}\right)^{\mathrm{T}}$,

$\mathbf{b}_{k}=\left(b_{k 1}, b_{k 2}, \ldots, b_{k n}\right)^{\mathrm{T}}$,

$\mathbf{c}_{k}=\left(c_{k 1}, c_{k 2}, \ldots, c_{k n}\right)^{\mathrm{T}}$

and the corresponding coefficients slowly varying with time are determined by

$\dot{\mathbf{a}}_{0}=\mathbf{F}_{0}\left(\mathbf{a}_{0}, \mathbf{b}, \mathbf{c}\right)$,

$\dot{\mathbf{b}}=-\Omega \mathbf{k}_{1} \mathbf{c}+\mathbf{F}_{1}\left(\mathbf{a}_{0}, \mathbf{b}, \mathbf{c}\right)$,

$\dot{\mathbf{c}}=\Omega \mathbf{k}_{1} \mathbf{b}+\mathbf{F}_{2}\left(\mathbf{a}_{0}, \mathbf{b}, \mathbf{c}\right) ;$

where

$\mathbf{k}_{1}=\operatorname{diag}\left(\mathbf{I}_{n \times n}, 2 \mathbf{I}_{n \times n}, \ldots, N \mathbf{I}_{n \times n}\right)$,

$\mathbf{b}=\left(\mathbf{b}_{1}, \mathbf{b}_{2}, \ldots, \mathbf{b}_{N}\right)^{\mathrm{T}}$,

$\mathbf{c}=\left(\mathbf{c}_{1}, \mathbf{c}_{2}, \ldots, \mathbf{c}_{N}\right)^{\mathrm{T}}$,

$\mathbf{F}_{1}=\left(\mathbf{F}_{11}, \mathbf{F}_{12}, \ldots, \mathbf{F}_{1 N}\right)^{\mathrm{T}}$,

$\mathbf{F}_{2}=\left(\mathbf{F}_{21}, \mathbf{F}_{22}, \ldots, \mathbf{F}_{2 N}\right)^{\mathrm{T}}$

for $N=1,2, \ldots, \infty$.

and

$\mathbf{F}_{0}\left(\mathbf{a}_{0}, \mathbf{b}, \mathbf{c}\right)=\frac{1}{T} \int_{0}^{T} \mathbf{f}\left(\mathbf{x}^{*}, \mathbf{p}\right) d t$

$\mathbf{F}_{1 k}\left(\mathbf{a}_{0}, \mathbf{b}, \mathbf{c}\right)=\frac{2}{T} \int_{0}^{T} \mathbf{f}\left(\mathbf{x}^{*}, \mathbf{p}\right) \cos (k \Omega t) d t$,

$\mathbf{F}_{2 k}\left(\mathbf{a}_{0}, \mathbf{b}, \mathbf{c}\right)=\frac{2}{T} \int_{0}^{T} \mathbf{f}\left(\mathbf{x}^{*}, \mathbf{p}\right) \sin (k \Omega t) d t ;$

for $k=1,2, \ldots, N$.

Equation (4) becomes

$\dot{\mathbf{z}}=\mathbf{f}(\mathbf{z})$

where

$\mathbf{z}=\left(\mathbf{a}_{0}, \mathbf{b}, \mathbf{c}\right)^{\mathrm{T}}$,

$\mathbf{f}=\left(\mathbf{F}_{0},-\Omega \mathbf{k}_{1} \mathbf{c}+\mathbf{F}_{1}, \Omega \mathbf{k}_{1} \mathbf{b}+\mathbf{F}_{2}\right)^{\mathrm{T}}$.

If the equilibrium $\mathbf{z}^{*}$ of Eq. (7) (i.e., $\mathbf{f}\left(\mathbf{z}^{*}\right)=\mathbf{0}$ ) exists, then the approximate analytical solution of the periodic flow exists in Eq. (2). In vicinity of equilibrium $\mathbf{z}^{*}$, with $\mathbf{z}=\mathbf{z}^{*}+\Delta \mathbf{z}$ the linearized equation of Eq. (4) is

$\Delta \dot{\mathbf{z}}=D \mathbf{f}\left(\mathbf{z}^{*}\right) \Delta \mathbf{z}$ and the eigenvalue analysis of equilibrium $\mathbf{z}^{*}$ is given by

$\left|D \mathbf{f}\left(\mathbf{z}^{*}\right)-\lambda \mathbf{I}_{n(2 N+1) \times n(2 N+1)}\right|=0$

where Df( $\left(\mathbf{z}^{*}\right)=\partial \mathbf{f}(\mathbf{z}) /\left.\partial \mathbf{z}\right|_{\mathbf{z}^{*}}$. Thus, the stability and bifurcation of the periodic solution can be classified through eigenvalues of Df( $\left.\mathbf{z}^{*}\right)$ with

$\left(n_{1}, n_{2}, n_{3} \mid n_{4}, n_{5}, n_{6}\right)$

where $n_{1}$ is the total number of negative real eigenvalues, $n_{2}$ is the total number of positive real eigenvalues, $n_{3}$ is the total number of zero real eigenvalues; $n_{4}$ is the total pair number of complex eigenvalues with negative real parts, $n_{5}$ is the total pair number of complex eigenvalues with positive real parts, $n_{6}$ is the total pair number of complex eigenvalues with zero real parts.

(i) If all eigenvalues of the equilibrium possess negative real parts, the approximate periodic solution is stable.

(ii) If at least one of eigenvalues of the equilibrium possesses positive real part, the approximate periodic solution is unstable.

(iii) The boundaries between stable and unstable equilibriums with higher order singularity give the bifurcation and stability conditions with higher order singularity.

Proof The proof can be referred to Luo [39].

If the Hopf bifurcation of equilibriums of Eq. (4) occurs, there is a periodic solution of coefficients in Eq. (2) with a frequency $\omega$. Thus, the periodic solution of coefficients can be expressed as

$\mathbf{a}_{0}(t)=\mathbf{A}_{00}(t)+\sum_{m=1}^{M} \mathbf{A}_{0 m}^{(1)}(t) \cos (m \omega t)+\mathbf{A}_{0 m}^{(2)}(t) \sin (m \omega t)$.

$\mathbf{b}_{k}(t)=\mathbf{B}_{k 0}(t)+\sum_{m=1}^{M} \mathbf{B}_{k m}^{(1)}(t) \cos (m \omega t)+\mathbf{B}_{k m}^{(2)}(t) \sin (m \omega t)$,

$\mathbf{c}_{k}(t)=\mathbf{C}_{k 0}(t)+\sum_{m=1}^{M} \mathbf{C}_{k m}^{(1)}(t) \cos (m \omega t)+\mathbf{C}_{k m}^{(2)}(t) \sin (m \omega t)$,

Substitution of Eq. (12) into Eq. (2) gives

$$
\begin{aligned}
\mathbf{x}^{*}(t)= & \mathbf{A}_{00}(t)+\sum_{m=1}^{M} \mathbf{A}_{0 m}^{(1)}(t) \cos (m \omega t)+\mathbf{A}_{0 m}^{(2)}(t) \sin (m \omega t) \\
& +\sum_{k=1}^{N} \mathbf{B}_{k 0}(t) \cos (k \Omega t)+\mathbf{C}_{k 0}(t) \sin (k \Omega t) \\
& +\sum_{k=1}^{N} \sum_{m=1}^{M} \mathbf{B}_{k m}^{(1)}(t) \cos (m \omega t) \cos (k \Omega t) \\
& +\mathbf{B}_{k m}^{(2)}(t) \sin (m \omega t) \cos (k \Omega t)
\end{aligned}
$$




$$
\begin{aligned}
& +\sum_{k=1}^{N} \sum_{m=1}^{M} \mathbf{C}_{k m}^{(1)}(t) \cos (m \omega t) \sin (k \Omega t) \\
& +\mathbf{C}_{k m}^{(2)}(t) \sin (m \omega t) \sin (k \Omega t) .
\end{aligned}
$$

If the new solution is still periodic with excitation period $T=2 \pi / \Omega$, then for specific $m$, we have

$m \omega=k \Omega$.

For this case, $k=1$ should be inserted because $k>1$ terms are already included in the Fourier series expression. Thus,

$m \omega=\Omega$.

For $m=1$, the period-1 flow is obtained and Eq. (13) will become Eq. (2). For the period- $m$ flow, we have

$$
\begin{aligned}
\mathbf{x}^{(m) *}(t)= & \mathbf{a}_{0}^{(m)}(t)+\sum_{k=1}^{N} \mathbf{b}_{k / m}(t) \cos \left(\frac{k}{m} \Omega t\right) \\
& +\mathbf{c}_{k / m}(t) \sin \left(\frac{k}{m} \Omega t\right) .
\end{aligned}
$$

If $m \omega \neq k \Omega$ for any $m$ and $k$, the periodic solutions will become quasi-periodic or chaotic instead of periodic. Herein, this case will not be discussed. If the approximate solution of period-1 motion possesses at least $N_{1}$ harmonic vector terms, then the total harmonic vector terms for period- $m$ motion should be $N \geq m N_{1}$. Similarly, the period- $m$ flows in nonlinear dynamical systems can be discussed.

Theorem 2 Consider a nonlinear dynamical system in Eq. (1). If such a dynamical system has a period-m flow $\mathbf{x}^{(m) *}(t)$ with finite norm $\left\|\mathbf{x}^{(m) *}\right\|$ and period $T=2 \pi / \Omega$, there is an analytical expression for the period-m flow of Eq. (1) in the form of

$$
\begin{aligned}
\mathbf{x}^{(m) *}(t)= & \mathbf{a}_{0}^{(m)}(t)+\sum_{k=1}^{N} \mathbf{b}_{k / m}(t) \cos \left(\frac{k}{m} \Omega t\right) \\
& +\mathbf{c}_{k / m}(t) \sin \left(\frac{k}{m} \Omega t\right)
\end{aligned}
$$

with for $k=1,2, \ldots, N$

$$
\begin{aligned}
& \mathbf{a}_{0}^{(m)}=\left(a_{01}^{(m)}, a_{02}^{(m)}, \ldots, a_{0 n}^{(m)}\right)^{\mathrm{T}}, \\
& \mathbf{b}_{k / m}=\left(b_{k / m 1}, b_{k / m 2}, \ldots, b_{k / m n}\right)^{\mathrm{T}}, \\
& \mathbf{c}_{k / m}=\left(c_{k / m 1}, c_{k / m 2}, \ldots, c_{k / m n}\right)^{\mathrm{T}}
\end{aligned}
$$

and the corresponding coefficients slowly varying with time are determined by

$$
\begin{aligned}
\dot{\mathbf{a}}_{0}^{(m)} & =\mathbf{F}_{0}^{(m)}\left(\mathbf{a}_{0}^{(m)}, \mathbf{b}^{(m)}, \mathbf{c}^{(m)}\right), \\
\dot{\mathbf{b}}^{(m)} & =-\frac{\Omega}{m} \mathbf{k}_{1} \mathbf{c}^{(m)}+\mathbf{F}_{1}^{(m)}\left(\mathbf{a}_{0}^{(m)}, \mathbf{b}^{(m)}, \mathbf{c}^{(m)}\right), \\
\dot{\mathbf{c}}^{(m)} & =\frac{\Omega}{m} \mathbf{k}_{1} \mathbf{b}^{(m)}+\mathbf{F}_{2}^{(m)}\left(\mathbf{a}_{0}^{(m)}, \mathbf{b}^{(m)}, \mathbf{c}^{(m)}\right)
\end{aligned}
$$

where

$$
\begin{aligned}
& \mathbf{k}_{1}=\operatorname{diag}\left(\mathbf{I}_{n \times n}, 2 \mathbf{I}_{n \times n}, \ldots, N \mathbf{I}_{n \times n}\right), \\
& \mathbf{b}^{(m)}=\left(\mathbf{b}_{1 / m}, \mathbf{b}_{2 / m}, \ldots, \mathbf{b}_{N / m}\right)^{\mathrm{T}}, \\
& \mathbf{c}^{(m)}=\left(\mathbf{c}_{1 / m}, \mathbf{c}_{2 / m}, \ldots, \mathbf{c}_{N / m}\right)^{\mathrm{T}}, \\
& \mathbf{F}_{1}^{(m)}=\left(\mathbf{F}_{11}^{(m)}, \mathbf{F}_{12}^{(m)}, \ldots, \mathbf{F}_{1 N}^{(m)}\right)^{\mathrm{T}}, \\
& \mathbf{F}_{2}^{(m)}=\left(\mathbf{F}_{21}^{(m)}, \mathbf{F}_{22}^{(m)}, \ldots, \mathbf{F}_{2 N}^{(m)}\right)^{\mathrm{T}} \\
& \text { for } \quad N=1,2, \ldots, \infty ;
\end{aligned}
$$

and

$$
\mathbf{F}_{0}^{(m)}\left(\mathbf{a}_{0}^{(m)}, \mathbf{b}^{(m)}, \mathbf{c}^{(m)}\right)=\frac{1}{m T} \int_{0}^{m T} \mathbf{f}\left(\mathbf{x}^{(m) *}, \mathbf{p}\right) d t
$$

$\mathbf{F}_{1 k}^{(m)}\left(\mathbf{a}_{0}^{(m)}, \mathbf{b}^{(m)}, \mathbf{c}^{(m)}\right)=\frac{2}{m T} \int_{0}^{m T} \mathbf{f}\left(\mathbf{x}^{(m) *}, \mathbf{p}\right) \cos \left(\frac{k}{m} \Omega t\right) d t$,

$\mathbf{F}_{2 k}^{(m)}\left(\mathbf{a}_{0}^{(m)}, \mathbf{b}^{(m)}, \mathbf{c}^{(m)}\right)=\frac{2}{m T} \int_{0}^{m T} \mathbf{f}\left(\mathbf{x}^{(m) *}, \mathbf{p}\right) \sin \left(\frac{k}{m} \Omega t\right) d t$

for $k=1,2, \ldots, N$.

Equation (19) becomes

$\dot{\mathbf{z}}^{(m)}=\mathbf{f}^{(m)}\left(\mathbf{z}^{(m)}\right)$

where

$\mathbf{z}^{(m)}=\left(\mathbf{a}_{0}^{(m)}, \mathbf{b}^{(m)}, \mathbf{c}^{(m)}\right)^{\mathrm{T}}$

$\mathbf{f}^{(m)}=\left(\mathbf{F}_{0}^{(m)},-\Omega \mathbf{k}_{1} \mathbf{c}^{(m)} / m+\mathbf{F}_{1}^{(m)}, \Omega \mathbf{k}_{1} \mathbf{b}^{(m)} / m+\mathbf{F}_{2}^{(m)}\right)^{\mathrm{T}}$

If equilibrium $\mathbf{z}^{(m) *}$ of Eq. (22) (i.e., $\left.\mathbf{f}^{(m)}\left(\mathbf{z}^{(m) *}\right)=\mathbf{0}\right)$ exists, then the approximate analytical solution of the period- $m$ flow exists in Eq. (17). In vicinity of equilibrium $\mathbf{z}^{(m) *}$, with $\mathbf{z}^{(m)}=\mathbf{z}^{(m) *}+\Delta \mathbf{z}^{(m)}$, the linearized equation of Eq. (22) is

$\Delta \dot{\mathbf{z}}^{(m)}=D \mathbf{f}^{(m)}\left(\mathbf{z}^{(m) *}\right) \Delta \mathbf{z}^{(m)}$

and the eigenvalue analysis of equilibrium $\mathbf{z}^{*}$ is given by

$\left|D \mathbf{f}^{(m)}\left(\mathbf{z}^{(m) *}\right)-\lambda \mathbf{I}_{n(2 N+1) \times n(2 N+1)}\right|=0$

where D $\mathbf{f}^{(m)}\left(\mathbf{z}^{(m) *}\right)=\partial \mathbf{f}^{(m)}\left(\mathbf{z}^{(m)}\right) /\left.\partial \mathbf{z}^{(m)}\right|_{\mathbf{z}^{(m) *} \text {. The stability }}$ and bifurcation of such a periodic solution can be classified by eigenvalues of $D \mathbf{f}^{(m)}\left(\mathbf{z}^{(m) *}\right)$ with

$\left(n_{1}, n_{2}, n_{3} \mid n_{4}, n_{5}, n_{6}\right)$

where $n_{1}$ is the total number of negative real eigenvalues, $n_{2}$ is the total number of positive real eigenvalues, $n_{3}$ is the total number of zero real eigenvalues; $n_{4}$ is the total pair number of complex eigenvalues with negative real parts, $n_{5}$ is the total pair number of complex eigenvalues with positive real parts, $n_{6}$ is the total pair number of complex eigenvalues with zero real parts. 
(i) If all eigenvalues of the equilibrium possess negative real parts, the approximate periodic solution is stable.

(ii) If at least one of eigenvalues of the equilibrium possesses positive real part, the approximate periodic solution is unstable.

(iii) The boundaries between stable and unstable equilibriums with higher order singularity give bifurcation and stability conditions with higher order singularity.

Proof The proof can be referred to Luo [39].

If $m \rightarrow \infty$ with $N \rightarrow \infty$, Eq. (17) will give the analytical expression of chaos in dynamical systems in Eq. (1). The route from the periodic flow to chaos is through the Hopf bifurcation.

\subsection{Periodically forced nonlinear systems}

Periodic flows in periodically forced nonlinear dynamical systems will be presented. Suppose a periodically forced nonlinear system with an external period $T=2 \pi / \Omega$ has a periodic flow, then such a periodic flow can be expressed by a Fourier series.

Theorem 3 Consider a periodically forced, nonlinear dynamical system as

$\dot{\mathbf{x}}=\mathbf{F}(\mathbf{x}, t, \mathbf{p}) \in \mathbf{R}^{n}$

where $\mathbf{F}(\mathbf{x}, t, \mathbf{p})$ is a $C^{r}$-continuous nonlinear function vector $(r \geq 1)$ with a forcing period $T=2 \pi / \Omega$. If there is a periodic flow $\mathbf{x}^{*}(t)$ with finite norm $\left\|\mathbf{x}^{*}\right\|$, an analytical expression for the periodic flow of Eq. (27) is in the form of

$\mathbf{x}^{*}(t)=\mathbf{a}_{0}(t)+\sum_{k=1}^{N} \mathbf{b}_{k}(t) \cos (k \Omega t)+\mathbf{c}_{k}(t) \sin (k \Omega t)$

with

$\mathbf{a}_{0}=\left(a_{01}, a_{02}, \ldots, a_{0 n}\right)^{\mathrm{T}}$,

$\mathbf{b}_{k}=\left(b_{k 1}, b_{k 2}, \ldots, b_{k n}\right)^{\mathrm{T}}$,

$\mathbf{c}_{k}=\left(c_{k 1}, c_{k 2}, \ldots, c_{k n}\right)^{\mathrm{T}}$;

and the corresponding coefficients slowly varying with time are determined by

$\dot{\mathbf{a}}_{0}=\mathbf{F}_{0}\left(\mathbf{a}_{0}, \mathbf{b}, \mathbf{c}\right)$,

$\dot{\mathbf{b}}=-\Omega \mathbf{k}_{1} \mathbf{c}+\mathbf{F}_{1}\left(\mathbf{a}_{0}, \mathbf{b}, \mathbf{c}\right)$,

$\dot{\mathbf{c}}=\Omega \mathbf{k}_{1} \mathbf{b}+\mathbf{F}_{2}\left(\mathbf{a}_{0}, \mathbf{b}, \mathbf{c}\right)$;

where

$\mathbf{k}_{1}=\operatorname{diag}\left(\mathbf{I}_{n \times n}, 2 \mathbf{I}_{n \times n}, \ldots, N \mathbf{I}_{n \times n}\right)$,

$\mathbf{b}=\left(\mathbf{b}_{1}, \mathbf{b}_{2}, \ldots, \mathbf{b}_{N}\right)^{\mathrm{T}}$,

$\mathbf{c}=\left(\mathbf{c}_{1}, \mathbf{c}_{2}, \ldots, \mathbf{c}_{N}\right)^{\mathrm{T}}$,

$\mathbf{F}_{1}=\left(\mathbf{F}_{11}, \mathbf{F}_{12}, \ldots, \mathbf{F}_{1 N}\right)^{\mathrm{T}}$,

$\mathbf{F}_{2}=\left(\mathbf{F}_{21}, \mathbf{F}_{22}, \ldots, \mathbf{F}_{2 N}\right)^{\mathrm{T}}$

for $N=1,2, \ldots, \infty$; and for $k=1,2, \ldots, N$

$\mathbf{F}_{0}\left(\mathbf{a}_{0}, \mathbf{b}, \mathbf{c}\right)=\frac{1}{T} \int_{0}^{T} \mathbf{F}\left(\mathbf{x}^{*}, t, \mathbf{p}\right) d t$

$\mathbf{F}_{1 k}\left(\mathbf{a}_{0}, \mathbf{b}, \mathbf{c}\right)=\frac{2}{T} \int_{0}^{T} \mathbf{F}\left(\mathbf{x}^{*}, t, \mathbf{p}\right) \cos (k \Omega t) d t$,

$\mathbf{F}_{2 k}\left(\mathbf{a}_{0}, \mathbf{b}, \mathbf{c}\right)=\frac{2}{T} \int_{0}^{T} \mathbf{F}\left(\mathbf{x}^{*}, t, \mathbf{p}\right) \sin (k \Omega t) d t$.

Equation (30) becomes

$\dot{\mathbf{z}}=\mathbf{f}(\mathbf{z})$

where

$\mathbf{z}=\left(\mathbf{a}_{0}, \mathbf{b}, \mathbf{c}\right)^{\mathrm{T}}$,

$\mathbf{f}=\left(\mathbf{F}_{0},-\Omega \mathbf{k}_{1} \mathbf{c}+\mathbf{F}_{1}, \Omega \mathbf{k}_{1} \mathbf{c}+\mathbf{F}_{2}\right)^{\mathrm{T}}$

If equilibrium $\mathbf{z}^{*}$ of Eq. (33) (i.e., $\mathbf{f}\left(\mathbf{z}^{*}\right)=\mathbf{0}$ ) exists, then the approximate analytical solution of the periodic flow exists in Eq. (28). In vicinity of equilibrium $\mathbf{z}^{*}$, with $\mathbf{z}=\mathbf{z}^{*}+\Delta \mathbf{z}$ the linearized equation of Eq. (33) is

$\Delta \dot{\mathbf{z}}=D \mathbf{f}\left(\mathbf{z}^{*}\right) \Delta \mathbf{z}$

and the eigenvalue analysis of equilibrium $\mathbf{z}^{*}$ is given by

$\left|D \mathbf{f}\left(\mathbf{z}^{*}\right)-\lambda \mathbf{I}_{n(2 N+1) \times n(2 N+1)}\right|=0$

where Df $\left(\mathbf{z}^{*}\right)=\partial \mathbf{f}(\mathbf{z}) /\left.\partial \mathbf{z}\right|_{\mathbf{z}^{*}}$. Thus, the stability and bifurcation of the periodic solution can be classified by eigenvalues of $\mathbf{f}\left(\mathbf{z}^{*}\right)$ with

$\left(n_{1}, n_{2}, n_{3} \mid n_{4}, n_{5}, n_{6}\right)$.

(i) If all eigenvalues of the equilibrium possess negative real parts, the approximate periodic solution is stable.

(ii) If at least one of eigenvalues of the equilibrium possesses positive real part, the approximate periodic solution is unstable.

(iii) The boundaries between stable and unstable equilibriums with higher order singularity give bifurcation and stability conditions with higher order singularity.

Proof The proof can be referred to Luo [39].

As similar to the autonomous system, period- $m$ flows in the periodically forced, nonlinear dynamical system in Eq. (27) can be discussed.

Theorem 4 Consider a periodically forced, nonlinear dynamical system in Eq. (27) with a forcing period $T=2 \pi / \Omega$. If there is a period- $m$ flow $\mathbf{x}^{(m) *}(t)$ with finite norm $\left\|\mathbf{x}^{(m) *}\right\|$, an analytical expression for the period-m flow of Eq. (27) is 


$$
\begin{aligned}
\mathbf{x}^{(m) *}(t)= & \mathbf{a}_{0}^{(m)}(t)+\sum_{k=1}^{N} \mathbf{b}_{k / m}(t) \cos \left(\frac{k}{m} \Omega t\right) \\
& +\mathbf{c}_{k / m}(t) \sin \left(\frac{k}{m} \Omega t\right)
\end{aligned}
$$

with for $k=1,2, \ldots, N$

$\mathbf{a}_{0}^{(m)}=\left(a_{01}^{(m)}, a_{02}^{(m)}, \ldots, a_{0 n}^{(m)}\right)^{\mathrm{T}}$,

$\mathbf{b}_{k / m}=\left(b_{k / m 1}, b_{k / m 2}, \ldots, b_{k / m n}\right)^{\mathrm{T}}$,

$\mathbf{c}_{k / m}=\left(c_{k / m 1}, c_{k / m 2}, \ldots, c_{k / m n}\right)^{\mathrm{T}}$

and the corresponding coefficients slowly varying with time are determined by

$\dot{\mathbf{a}}_{0}^{(m)}=\mathbf{F}_{0}^{(m)}\left(\mathbf{a}_{0}^{(m)}, \mathbf{b}^{(m)}, \mathbf{c}^{(m)}\right)$,

$\dot{\mathbf{b}}^{(m)}=-\frac{\Omega}{m} \mathbf{k}_{1} \mathbf{c}^{(m)}+\mathbf{F}_{1}^{(m)}\left(\mathbf{a}_{0}^{(m)}, \mathbf{b}^{(m)}, \mathbf{c}^{(m)}\right)$,

$\dot{\mathbf{c}}^{(m)}=\frac{\Omega}{m} \mathbf{k}_{1} \mathbf{b}^{(m)}+\mathbf{F}_{2}^{(m)}\left(\mathbf{a}_{0}^{(m)}, \mathbf{b}^{(m)}, \mathbf{c}^{(m)}\right)$

where for $N=1,2, \ldots, \infty$

$\mathbf{k}_{1}=\operatorname{diag}\left(\mathbf{I}_{n \times n}, 2 \mathbf{I}_{n \times n}, \ldots, N \mathbf{I}_{n \times n}\right)$,

$\mathbf{b}^{(m)}=\left(\mathbf{b}_{1 / m}, \mathbf{b}_{2 / m}, \ldots, \mathbf{b}_{N / m}\right)^{\mathrm{T}}$,

$\mathbf{c}^{(m)}=\left(\mathbf{c}_{1 / m}, \mathbf{c}_{2 / m}, \ldots, \mathbf{c}_{N / m}\right)^{\mathrm{T}}$,

$\mathbf{F}_{1}^{(m)}=\left(\mathbf{F}_{11}^{(m)}, \mathbf{F}_{12}^{(m)}, \ldots, \mathbf{F}_{1 N}^{(m)}\right)^{\mathrm{T}}$,

$\mathbf{F}_{2}^{(m)}=\left(\mathbf{F}_{21}^{(m)}, \mathbf{F}_{22}^{(m)}, \ldots, \mathbf{F}_{2 N}^{(m)}\right)^{\mathrm{T}}$

and

$$
\begin{aligned}
& \mathbf{F}_{0}^{(m)}\left(\mathbf{a}_{0}^{(m)}, \mathbf{b}^{(m)}, \mathbf{c}^{(m)}\right)=\frac{1}{m T} \int_{0}^{m T} \mathbf{f}\left(\mathbf{x}^{(m) *}, t, \mathbf{p}\right) d t \\
& \mathbf{F}_{1 k}^{(m)}\left(\mathbf{a}_{0}^{(m)}, \mathbf{b}^{(m)}, \mathbf{c}^{(m)}\right) \\
& =\frac{2}{m T} \int_{0}^{m T} \mathbf{f}\left(\mathbf{x}^{(m) *}, t, \mathbf{p}\right) \cos \left(\frac{k}{m} \Omega t\right) d t \\
& \mathbf{F}_{2 k}^{(m)}\left(\mathbf{a}_{0}^{(m)}, \mathbf{b}^{(m)}, \mathbf{c}^{(m)}\right) \\
& =\frac{2}{m T} \int_{0}^{m T} \mathbf{f}\left(\mathbf{x}^{(m) *}, t, \mathbf{p}\right) \sin \left(\frac{k}{m} \Omega t\right) d t \\
& \text { for } k=1,2, \ldots, N .
\end{aligned}
$$

Equation (40) becomes

$\dot{\mathbf{z}}^{(m)}=\mathbf{f}^{(m)}\left(\mathbf{z}^{(m)}\right)$

where

$$
\begin{aligned}
\mathbf{z}^{(m)}= & \left(\mathbf{a}_{0}^{(m)}, \mathbf{b}^{(m)}, \mathbf{c}^{(m)}\right)^{\mathrm{T}} \\
\mathbf{f}^{(m)}= & \left(\mathbf{F}_{0}^{(m)},-\Omega \mathbf{k}_{1} \mathbf{c}^{(m)} / m+\mathbf{F}_{1}^{(m)}\right. \\
& \left.\Omega \mathbf{k}_{1} \mathbf{b}^{(m)} / m+\mathbf{F}_{2}^{(m)}\right)^{\mathrm{T}}
\end{aligned}
$$

If equilibrium $\mathbf{z}^{(m) *}$ of Eq. (43) (i.e., $\left.\mathbf{f}^{(m)}\left(\mathbf{z}^{(m) *}\right)=\mathbf{0}\right)$ exists, then the approximate analytical solution of the period-m flow exists in Eq. (38). In vicinity of equilibrium $\mathbf{z}^{(m) *}$, with $\mathbf{z}^{(m)}=\mathbf{z}^{(m) *}+\Delta \mathbf{z}^{(m)}$, the linearized equation of Eq. (43) is

$\Delta \dot{\mathbf{z}}^{(m)}=D \mathbf{f}^{(m)}\left(\mathbf{z}^{(m) *}\right) \Delta \mathbf{z}^{(m)}$

and the eigenvalue analysis of equilibrium $\mathbf{z}^{*}$ is given by

$\left|D \mathbf{f}^{(m)}\left(\mathbf{z}^{(m) *}\right)-\lambda \mathbf{I}_{n(2 N+1) \times n(2 N+1)}\right|=0$

where D $\mathbf{f}^{(m)}\left(\mathbf{z}^{(m) *}\right)=\partial \mathbf{f}^{(m)}\left(\mathbf{z}^{(m)}\right) /\left.\partial \mathbf{z}^{(m)}\right|_{\mathbf{z}^{(m) *} \text {. The stability }}$ and bifurcation of the periodic solution can be classified by the eigenvalues of $D \mathbf{f}^{(m)}\left(\mathbf{z}^{(m) *}\right)$ with

$\left(n_{1}, n_{2}, n_{3} \mid n_{4}, n_{5}, n_{6}\right)$

(i) If all eigenvalues of the equilibrium possess negative real parts, the approximate periodic solution is stable.

(ii) If at least one of eigenvalues of the equilibrium possesses positive real part, the approximate periodic solution is unstable.

(iii) The boundaries between stable and unstable equilibriums with higher order singularity give bifurcation and stability conditions with higher order singularity.

Proof The proof can be referred to Luo [39].

If $m \rightarrow \infty$ with $N \rightarrow \infty$, Eq. (38) will give the analytical expression of chaos in periodically forced nonlinear dynamical systems in Eq. (27).

\section{Nonlinear vibration systems without time-delay}

In this section, the analytical solutions for nonlinear vibration systems are presented owing to extensive application in engineering, and the local stability and bifurcation theory will be applied to determine the stability and bifurcation of approximate solutions of nonlinear vibration systems.

\subsection{Free vibration systems}

Periodic motions in nonlinear vibration systems will be presented herein. If such a vibration system has periodic motion with period $T=2 \pi / \Omega$, then such periodic motions can be expressed by the Fourier series.

Theorem 5 Consider a nonlinear dynamical system as

$\ddot{\mathbf{x}}=\mathbf{f}(\mathbf{x}, \dot{\mathbf{x}}, \mathbf{p}) \in \mathbf{R}^{n}$

where $\mathbf{f}(\mathbf{x}, \dot{\mathbf{x}}, \mathbf{p})$ is a $C^{r}$-continuous nonlinear function vector $(r \geq 1)$. If such a dynamical system has a periodic motion $\mathbf{x}^{*}(t)$ with finite norm $\left\|\mathbf{x}^{*}\right\|$ and period $T=2 \pi / \Omega$, there is 
an analytical expression for the periodic motion of Eq. (48) in the form of

$\mathbf{x}^{*}(t)=\mathbf{a}_{0}(t)+\sum_{k=1}^{N} \mathbf{b}_{k}(t) \cos (k \Omega t)+\mathbf{c}_{k}(t) \sin (k \Omega t)$.

with

$\begin{aligned} \mathbf{a}_{0} & =\left(a_{01}, a_{02}, \ldots, a_{0 n}\right)^{\mathrm{T}}, \\ \mathbf{b}_{k} & =\left(b_{k 1}, b_{k 2}, \ldots, b_{k n}\right)^{\mathrm{T}}, \\ \mathbf{c}_{k} & =\left(c_{k 1}, c_{k 2}, \ldots, c_{k n}\right)^{\mathrm{T}}\end{aligned}$

and the corresponding coefficients slowly varying with time are determined by

$\ddot{\mathbf{a}}_{0}=\mathbf{F}_{0}\left(\mathbf{a}_{0}, \mathbf{b}, \mathbf{c}, \dot{\mathbf{a}}_{0}, \dot{\mathbf{b}}, \dot{\mathbf{c}}\right)$,

$\ddot{\mathbf{b}}=-2 \Omega \mathbf{k}_{1} \dot{\mathbf{c}}+\Omega^{2} \mathbf{k}_{2} \mathbf{b}+\mathbf{F}_{1}\left(\mathbf{a}_{0}, \mathbf{b}, \mathbf{c}, \dot{\mathbf{a}}_{0}, \dot{\mathbf{b}}, \dot{\mathbf{c}}\right)$,

$\ddot{\mathbf{c}}=2 \Omega \mathbf{k}_{1} \dot{\mathbf{b}}+\Omega^{2} \mathbf{k}_{2} \mathbf{c}+\mathbf{F}_{2}\left(\mathbf{a}_{0}, \mathbf{b}, \mathbf{c}, \dot{\mathbf{a}}_{0}, \dot{\mathbf{b}}, \dot{\mathbf{c}}\right) ;$

where

$\mathbf{k}_{1}=\operatorname{diag}\left(\mathbf{I}_{n \times n}, 2 \mathbf{I}_{n \times n}, \ldots, N \mathbf{I}_{n \times n}\right)$,

$\mathbf{k}_{2}=\operatorname{diag}\left(\mathbf{I}_{n \times n}, 2^{2} \mathbf{I}_{n \times n}, \ldots, N^{2} \mathbf{I}_{n \times n}\right)$,

$\mathbf{b}=\left(\mathbf{b}_{1}, \mathbf{b}_{2}, \ldots, \mathbf{b}_{N}\right)^{\mathrm{T}}$,

$\mathbf{c}=\left(\mathbf{c}_{1}, \mathbf{c}_{2}, \ldots, \mathbf{c}_{N}\right)^{\mathrm{T}}$,

$\mathbf{F}_{1}=\left(\mathbf{F}_{11}, \mathbf{F}_{12}, \ldots, \mathbf{F}_{1 N}\right)^{\mathrm{T}}$,

$\mathbf{F}_{2}=\left(\mathbf{F}_{21}, \mathbf{F}_{22}, \ldots, \mathbf{F}_{2 N}\right)^{\mathrm{T}}$

for $N=1,2, \ldots, \infty$.

and for $k=1,2, \ldots, N$

$\mathbf{F}_{0}\left(\mathbf{a}_{0}, \mathbf{b}, \mathbf{c}, \dot{\mathbf{a}}_{0}, \dot{\mathbf{b}}, \dot{\mathbf{c}}\right)=\frac{1}{T} \int_{0}^{T} \mathbf{f}\left(\mathbf{x}^{*}, \dot{\mathbf{x}}^{*}, \mathbf{p}\right) d t ;$

$\mathbf{F}_{1 k}\left(\mathbf{a}_{0}, \mathbf{b}, \mathbf{c}, \dot{\mathbf{a}}_{0}, \dot{\mathbf{b}}, \dot{\mathbf{c}}\right)=\frac{2}{T} \int_{0}^{T} \mathbf{f}\left(\mathbf{x}^{*}, \dot{\mathbf{x}}^{*}, \mathbf{p}\right) \cos (k \Omega t) d t$,

$\mathbf{F}_{2 k}\left(\mathbf{a}_{0}, \mathbf{b}, \mathbf{c}, \dot{\mathbf{a}}_{0}, \dot{\mathbf{b}}, \dot{\mathbf{c}}\right)=\frac{2}{T} \int_{0}^{T} \mathbf{f}\left(\mathbf{x}^{*}, \dot{\mathbf{x}}^{*}, \mathbf{p}\right) \sin (k \Omega t) d t$.

The state-space form of Eq. (51) is

$\dot{\mathbf{z}}=\mathbf{z}_{1} \quad$ and $\quad \dot{\mathbf{z}}_{1}=\mathbf{g}\left(\mathbf{z}, \mathbf{z}_{1}\right)$

where

$\mathbf{z}=\left(\mathbf{a}_{0}, \mathbf{b}, \mathbf{c}\right)^{\mathrm{T}}, \dot{\mathbf{z}}=\mathbf{z}_{1}$,

$\mathbf{g}=\left(\mathbf{F}_{0},-2 \Omega \mathbf{k}_{1} \dot{\mathbf{c}}+\Omega^{2} \mathbf{k}_{2} \mathbf{b}+\mathbf{F}_{1}, 2 \Omega \mathbf{k}_{1} \dot{\mathbf{b}}+\Omega^{2} \mathbf{k}_{2} \mathbf{c}+\mathbf{F}_{2}\right)^{\mathrm{T}}$.

An equivalent system of Eq. (54) is

$\dot{\mathbf{y}}=\mathbf{f}(\mathbf{y})$

where

$\mathbf{y}=\left(\mathbf{z}, \mathbf{z}_{1}\right)^{\mathrm{T}}$ and $\mathbf{f}=\left(\mathbf{z}_{1}, \mathbf{g}\right)^{\mathrm{T}}$
If equilibrium $\mathbf{y}^{*}$ of Eq. (56) (i.e., $\mathbf{f}\left(\mathbf{y}^{*}\right)=\mathbf{0}$ ) exists, then the approximate analytical solution of periodic motion of Eq. (48) exists in Eq. (49). In vicinity of equilibrium $\mathbf{y}^{*}$, with $\mathbf{y}=\mathbf{y}^{*}+\Delta \mathbf{y}$ the linearized equation of $E q$. (56) is

$\Delta \dot{\mathbf{y}}=D \mathbf{f}\left(\mathbf{y}^{*}\right) \Delta \mathbf{y}$

and the eigenvalue analysis of equilibrium $\mathbf{y}^{*}$ is given by

$\left|D \mathbf{f}\left(\mathbf{y}^{*}\right)-\lambda \mathbf{I}_{2 n(2 N+1) \times 2 n(2 N+1)}\right|=0$

where Df $\left(\mathbf{y}^{*}\right)=\partial \mathbf{f}(\mathbf{y}) /\left.\partial \mathbf{y}\right|_{\mathbf{y}^{*}}$. Thus, the stability and bifurcation of periodic solution can be classified by the eigenvalues of $D \mathbf{f}\left(\mathbf{y}^{*}\right)$ with

$\left(n_{1}, n_{2}, n_{3} \mid n_{4}, n_{5}, n_{6}\right)$

(i) If all eigenvalues of the equilibrium possess negative real parts, the approximate periodic solution is stable.

(ii) If at least one of eigenvalues of the equilibrium possesses positive real part, the approximate periodic solution is unstable.

(iii) The boundaries between stable and unstable equilibriums with higher order singularity give bifurcation and stability conditions with higher order singularity.

Proof The proof can be referred to Luo [39].

If the Hopf bifurcation of equilibriums of Eq. (51) occurs, there is a periodic solution of coefficients in Eq. (49) with a frequency $\omega$. As discussed from Eqs. (12)-(16), there is a period- $m$ motion as in Eq. (17). If $m \omega \neq k \Omega$ for any $m$ and $k$, the solution will be quasi-periodic or chaotic instead of periodic. Herein, the period- $m$ motions in nonlinear vibration systems will be discussed.

Theorem 6 Consider a nonlinear dynamical system in Eq. (48). If such a dynamical system has a period-m motion $\mathbf{x}^{(m) *}(t)$ with finite norm $\left\|\mathbf{x}^{(m) *}\right\|$ and period $T=2 \pi / \Omega$, there is an analytical expression for the period-m motion of Eq. (48) in the form of

$$
\begin{aligned}
\mathbf{x}^{(m) *}(t)= & \mathbf{a}_{0}^{(m)}(t)+\sum_{k=1}^{N} \mathbf{b}_{k / m}(t) \cos \left(\frac{k}{m} \Omega t\right) \\
& +\mathbf{c}_{k / m}(t) \sin \left(\frac{k}{m} \Omega t\right)
\end{aligned}
$$

with for $k=1,2, \ldots, N$

$$
\begin{aligned}
& \mathbf{a}_{0}^{(m)}=\left(a_{01}^{(m)}, a_{02}^{(m)}, \ldots, a_{0 n}^{(m)}\right)^{\mathrm{T}}, \\
& \mathbf{b}_{k / m}=\left(b_{k / m 1}, b_{k / m 2}, \ldots, b_{k / m n}\right)^{\mathrm{T}}, \\
& \mathbf{c}_{k / m}=\left(c_{k / m 1}, c_{k / m 2}, \ldots, c_{k / m n}\right)^{\mathrm{T}}
\end{aligned}
$$


and the corresponding coefficients slowly varying with time are determined by the following dynamical systems

$$
\begin{aligned}
\ddot{\mathbf{a}}_{0}^{(m)}= & \mathbf{F}_{0}^{(m)}\left(\mathbf{a}_{0}^{(m)}, \mathbf{b}^{(m)}, \mathbf{c}^{(m)}, \dot{\mathbf{a}}_{0}^{(m)}, \dot{\mathbf{b}}^{(m)}, \dot{\mathbf{c}}^{(m)}\right), \\
\ddot{\mathbf{b}}^{(m)}= & -2 \frac{\Omega}{m} \mathbf{k}_{1} \dot{\mathbf{c}}^{(m)}+\frac{\Omega^{2}}{m^{2}} \mathbf{k}_{2} \mathbf{b}^{(m)} \\
& +\mathbf{F}_{1}^{(m)}\left(\mathbf{a}_{0}^{(m)}, \mathbf{b}^{(m)}, \mathbf{c}^{(m)}, \dot{\mathbf{a}}_{0}^{(m)}, \dot{\mathbf{b}}^{(m)}, \dot{\mathbf{c}}^{(m)}\right), \\
\ddot{\mathbf{c}}^{(m)}= & 2 \frac{\Omega}{m} \mathbf{k}_{1} \dot{\mathbf{b}}^{(m)}+\frac{\Omega^{2}}{m^{2}} \mathbf{k}_{2} \mathbf{c}^{(m)} \\
& +\mathbf{F}_{2}^{(m)}\left(\mathbf{a}_{0}^{(m)}, \mathbf{b}^{(m)}, \mathbf{c}^{(m)}, \dot{\mathbf{a}}_{0}^{(m)}, \dot{\mathbf{b}}^{(m)}, \dot{\mathbf{c}}^{(m)}\right)
\end{aligned}
$$

where

$$
\begin{aligned}
& \mathbf{k}_{1}=\operatorname{diag}\left(\mathbf{I}_{n \times n}, 2 \mathbf{I}_{n \times n}, \ldots, N \mathbf{I}_{n \times n}\right), \\
& \mathbf{k}_{2}=\operatorname{diag}\left(\mathbf{I}_{n \times n}, 2^{2} \mathbf{I}_{n \times n}, \ldots, N^{2} \mathbf{I}_{n \times n}\right), \\
& \mathbf{b}^{(m)}=\left(\mathbf{b}_{1 / m}, \mathbf{b}_{2 / m}, \ldots, \mathbf{b}_{N / m}\right)^{\mathrm{T}}, \\
& \mathbf{c}^{(m)}=\left(\mathbf{c}_{1 / m}, \mathbf{c}_{2 / m}, \ldots, \mathbf{c}_{N / m}\right)^{\mathrm{T}}, \\
& \mathbf{F}_{1}^{(m)}=\left(\mathbf{F}_{11}^{(m)}, \mathbf{F}_{12}^{(m)}, \ldots, \mathbf{F}_{1 N}^{(m)}\right)^{\mathrm{T}}, \\
& \mathbf{F}_{2}^{(m)}=\left(\mathbf{F}_{21}^{(m)}, \mathbf{F}_{22}^{(m)}, \ldots, \mathbf{F}_{2 N}^{(m)}\right)^{\mathrm{T}} \\
& \text { for } \quad N=1,2, \ldots, \infty . \\
& \text { and for } k=1,2, \ldots, N \\
& \mathbf{F}_{0}^{(m)}\left(\mathbf{a}_{0}^{(m)}, \mathbf{b}^{(m)}, \mathbf{c}^{(m)}, \dot{\mathbf{a}}_{0}^{(m)}, \dot{\mathbf{b}}^{(m)}, \dot{\mathbf{c}}^{(m)}\right) \\
& \quad=\frac{1}{m T} \int_{0}^{m T} \mathbf{f}\left(\mathbf{x}^{(m) *}, \dot{\mathbf{x}}^{(m) *}, \mathbf{p}\right) d t ; \\
& \mathbf{F}_{1 k}^{(m)}\left(\mathbf{a}_{0}^{(m)}, \mathbf{b}^{(m)}, \mathbf{c}^{(m)}, \dot{\mathbf{a}}_{0}^{(m)}, \dot{\mathbf{b}}^{(m)}, \dot{\mathbf{c}}^{(m)}\right) \\
& \quad=\frac{2}{m T} \int_{0}^{m T} \mathbf{f}\left(\mathbf{x}^{(m) *}, \dot{\mathbf{x}}^{(m) *}, \mathbf{p}\right) \cos \left(\frac{k}{m} \Omega t\right) d t, \\
& \quad \mathbf{F}_{2 k}^{(m)}\left(\mathbf{a}_{0}^{(m)}, \mathbf{b}^{(m)}, \mathbf{c}^{(m)}, \dot{\mathbf{a}}_{0}^{(m)}, \dot{\mathbf{b}}^{(m)}, \dot{\mathbf{c}}^{(m)}\right) \\
& \quad \int_{m T}^{m T} \mathbf{f}\left(\mathbf{x}^{(m) *}, \dot{\mathbf{x}}^{(m) *}, \mathbf{p}\right) \sin \left(\frac{k}{m} \Omega t\right) d t .
\end{aligned}
$$

The state-space form of Eq. (63) is

$$
\dot{\mathbf{z}}^{(m)}=\mathbf{z}_{1}^{(m)} \text { and } \dot{\mathbf{z}}_{1}^{(m)}=\mathbf{g}^{(m)}\left(\mathbf{z}^{(m)}, \mathbf{z}_{1}^{(m)}\right)
$$

where

$$
\begin{aligned}
\mathbf{z}^{(m)}= & \left(\mathbf{a}_{0}^{(m)}, \mathbf{b}^{(m)}, \mathbf{c}^{(m)}\right)^{\mathrm{T}}, \dot{\mathbf{z}}^{(m)}=\mathbf{z}_{1}^{(m)} \\
\mathbf{g}^{(m)}= & \left(\mathbf{F}_{0}^{(m)},-2 \frac{\Omega}{m} \mathbf{k}_{1} \dot{\mathbf{c}}^{(m)}+\frac{\Omega^{2}}{m^{2}} \mathbf{k}_{2} \mathbf{b}^{(m)}+\mathbf{F}_{1}^{(m)},\right. \\
& \left.2 \frac{\Omega}{m} \mathbf{k}_{1} \dot{\mathbf{b}}^{(m)}+\frac{\Omega^{2}}{m^{2}} \mathbf{k}_{2} \mathbf{c}^{(m)}+\mathbf{F}_{2}^{(m)}\right)^{\mathrm{T}} .
\end{aligned}
$$

An equivalent system of $E q$. (66) is

$\dot{\mathbf{y}}^{(m)}=\mathbf{f}^{(m)}\left(\mathbf{y}^{(m)}\right)$

where

$\mathbf{y}^{(m)}=\left(\mathbf{z}^{(m)}, \mathbf{z}_{1}^{(m)}\right)^{\mathrm{T}}$ and $\mathbf{f}^{(m)}=\left(\mathbf{z}_{1}^{(m)}, \mathbf{g}^{(m)}\right)^{\mathrm{T}}$
If equilibrium $\mathbf{y}^{(m) *}$ of Eq. (68) (i.e. $\mathbf{f}^{(m)}\left(\mathbf{y}^{(m) *}\right)=\mathbf{0}$ ) exists, then the approimxate analytical solution of the period- $m$ motion exists in Eq. (61). In vicinity of equilibrium $\mathbf{y}^{(m) *}$, with $\mathbf{y}^{(m)}=\mathbf{y}^{(m) *}+\Delta \mathbf{y}^{(m)}$ the linearized equation of $E q$. (68) is

$\Delta \dot{\mathbf{y}}^{(m)}=D \mathbf{f}^{(m)}\left(\mathbf{y}^{(m) *}\right) \Delta \mathbf{y}^{(m)}$

and the eigenvalue analysis of equilibrium $\mathbf{y}^{(m) *}$ is given by

$$
\left|D \mathbf{f}^{(m)}\left(\mathbf{y}^{(m) *}\right)-\lambda \mathbf{I}_{2 n(2 N+1) \times 2 n(2 N+1)}\right|=0
$$

where Df $\mathbf{f}^{(m)}\left(\mathbf{y}^{(m) *}\right)=\partial \mathbf{f}^{(m)}\left(\mathbf{y}^{(m)}\right) /\left.\partial \mathbf{y}^{(m)}\right|_{\mathbf{y}^{(m) *} \text {. Thus, the }}$ stability and bifurcation of periodic solution can be classified by the eigenvalues of $D \mathbf{f}^{(m)}\left(\mathbf{y}^{(m) *}\right)$ with

$\left(n_{1}, n_{2}, n_{3} \mid n_{4}, n_{5}, n_{6}\right)$

(i) If all eigenvalues of the equilibrium possess negative real parts, the approximate periodic solution is stable.

(ii) If at least one of eigenvalues of the equilibrium possesses positive real part, the approximate periodic solution is unstable.

(iii) The boundaries between stable and unstable equilibriums with higher order singularity give bifurcation and stability conditions with higher order singularity.

Proof The proof can be referred to Luo [39].

If the Hopf bifurcation of period- $m$ motion occurs, the period-doubling solution of period- $m$ motion can be expressed by

$$
\begin{aligned}
\mathbf{x}^{*}(t)= & \mathbf{a}_{0}^{(2 m)}(t)+\sum_{k=1}^{N} \mathbf{b}_{k / 2 m}(t) \cos \left(\frac{k}{2 m} \Omega t\right) \\
& +\mathbf{c}_{k / 2 m}(t) \sin \left(\frac{k}{2 m} \Omega t\right)
\end{aligned}
$$

where for $k=1,2, \ldots, N$

$$
\begin{aligned}
\ddot{\mathbf{a}}_{0}^{(2 m)}= & \mathbf{F}_{0}^{(2 m)}\left(\dot{\mathbf{a}}_{0}^{(2 m)}, \dot{\mathbf{b}}^{(2 m)}, \dot{\mathbf{c}}^{(2 m)}, \mathbf{a}_{0}^{(2 m)}, \mathbf{b}^{(2 m)}, \mathbf{c}^{(2 m)}\right), \\
& \ddot{\mathbf{b}}^{(2 m)}+2 \frac{\Omega}{(2 m)} \mathbf{k}_{1} \dot{\mathbf{c}}^{(2 m)}-\frac{\Omega^{2}}{(2 m)^{2}} \mathbf{k}_{2} \mathbf{b}^{(2 m)} \\
= & \mathbf{F}_{1 k}^{(m)}\left(\dot{\mathbf{a}}_{0}^{(2 m)}, \dot{\mathbf{b}}^{(2 m)}, \dot{\mathbf{c}}^{(2 m)}, \mathbf{a}_{0}^{(2 m)}, \mathbf{b}^{(2 m)}, \mathbf{c}^{(2 m)}\right) \\
& \ddot{\mathbf{c}}^{(2 m)}-2 \frac{\Omega}{(2 m)} \mathbf{k}_{1} \dot{\mathbf{b}}^{(2 m)}-\frac{\Omega^{2}}{(2 m)^{2}} \mathbf{k}_{2} \mathbf{c}^{(2 m)} \\
= & \mathbf{F}_{2 k}^{(m)}\left(\dot{\mathbf{a}}_{0}^{(2 m)}, \dot{\mathbf{b}}^{(2 m)}, \dot{\mathbf{c}}^{(2 m)}, \mathbf{a}_{0}^{(2 m)}, \mathbf{b}^{(2 m)}, \mathbf{c}^{(2 m)}\right)
\end{aligned}
$$


where for $N=1,2, \ldots, \infty$.

$$
\begin{aligned}
& \mathbf{k}_{1}=\operatorname{diag}\left(\mathbf{I}_{n \times n}, 2 \mathbf{I}_{n \times n}, \ldots, N \mathbf{I}_{n \times n}\right), \\
& \mathbf{k}_{2}=\operatorname{diag}\left(\mathbf{I}_{n \times n}, 2^{2} \mathbf{I}_{n \times n}, \ldots, N^{2} \mathbf{I}_{n \times n}\right) ; \\
& \mathbf{b}^{(2 m)}=\left(\mathbf{b}_{1 / 2 m}, \mathbf{b}_{2 / 2 m}, \ldots, \mathbf{b}_{N / 2 m}\right)^{\mathrm{T}}, \\
& \mathbf{c}^{(2 m)}=\left(\mathbf{c}_{1 / 2 m}, \mathbf{c}_{2 / 2 m}, \ldots, \mathbf{c}_{N / 2 m}\right)^{\mathrm{T}} ; \\
& \mathbf{F}_{1}^{(2 m)}=\left(\mathbf{F}_{11}^{(2 m)}, \mathbf{F}_{12}^{(2 m)}, \ldots, \mathbf{F}_{1 N}^{(2 m)}\right)^{\mathrm{T}}, \\
& \mathbf{F}_{2}^{(2 m)}=\left(\mathbf{F}_{21}^{(2 m)}, \mathbf{F}_{22}^{(2 m)}, \ldots, \mathbf{F}_{2 N}^{(2 m)}\right)^{\mathrm{T}} .
\end{aligned}
$$

and for $k=1,2, \ldots, N$

$$
\begin{aligned}
& \mathbf{F}_{0}^{(2 m)}\left(\mathbf{a}_{0}^{(2 m)}, \mathbf{b}^{(2 m)}, \mathbf{c}^{(2 m)}, \dot{\mathbf{a}}_{0}^{(2 m)}, \dot{\mathbf{b}}^{(2 m)}, \dot{\mathbf{c}}^{(2 m)}\right) \\
& =\frac{1}{2 m T} \int_{0}^{2 m T} \mathbf{f}\left(\mathbf{x}^{(m) *}, \dot{\mathbf{x}}^{(m) *}, \mathbf{p}\right) d t ; \\
& \mathbf{F}_{1 k}^{(2 m)}\left(\mathbf{a}_{0}^{(2 m)}, \mathbf{b}^{(2 m)}, \mathbf{c}^{(2 m)}, \dot{\mathbf{a}}_{0}^{(2 m)}, \dot{\mathbf{b}}^{(2 m)}, \dot{\mathbf{c}}^{(2 m)}\right) \\
& =\frac{1}{m T} \int_{0}^{2 m T} \mathbf{f}\left(\mathbf{x}^{(m) *}, \dot{\mathbf{x}}^{(m) *}, \mathbf{p}\right) \cos \left(\frac{k}{2 m} \Omega t\right) d t, \\
& \mathbf{F}_{2 k}^{(2 m)}\left(\mathbf{a}_{0}^{(2 m)}, \mathbf{b}^{(2 m)}, \mathbf{c}^{(2 m)}, \dot{\mathbf{a}}_{0}^{(2 m)}, \dot{\mathbf{b}}^{(2 m)}, \dot{\mathbf{c}}^{(2 m)}\right) \\
& =\frac{1}{m T} \int_{0}^{2 m T} \mathbf{f}\left(\mathbf{x}^{(m) *}, \dot{\mathbf{x}}^{(m) *}, \mathbf{p}\right) \sin \left(\frac{k}{2 m} \Omega t\right) d t
\end{aligned}
$$

If the Hopf bifurcation of period- $2 m$ motion occurs again and again, the analytical expression for period- $2^{l} m$ motion can be expressed by

$$
\begin{aligned}
\mathbf{x}^{*}(t)= & \mathbf{a}_{0}^{\left(2^{l} m\right)}(t)+\sum_{k=1}^{N} \mathbf{b}_{k / 2^{l} m}(t) \cos \left(\frac{k}{2^{l} m} \Omega t\right) \\
& +\mathbf{c}_{k / 2^{l} m}(t) \sin \left(\frac{k}{2^{l} m} \Omega t\right) .
\end{aligned}
$$

where for $k=1,2, \ldots, N$

$$
\begin{aligned}
\ddot{\mathbf{a}}_{0}^{\left(2^{l} m\right)}= & \mathbf{F}_{0}^{\left(2^{l} m\right)}\left(\dot{\mathbf{a}}_{0}^{\left(2^{l} m\right)}, \dot{\mathbf{b}}^{\left(2^{l} m\right)}, \dot{\mathbf{c}}^{\left(2^{l} m\right)}, \mathbf{a}_{0}^{\left(2^{l} m\right)}, \mathbf{b}^{\left(2^{l} m\right)}, \mathbf{c}^{\left(2^{l} m\right)}\right), \\
& \ddot{\mathbf{b}}^{\left(2^{l} m\right)}+2 \frac{\Omega}{2^{l} m} \mathbf{k}_{1} \dot{\mathbf{c}}^{\left(2^{l} m\right)}-\frac{\Omega^{2}}{\left(2^{l} m\right)^{2}} \mathbf{k}_{2} \mathbf{b}^{\left(2^{l} m\right)} \\
= & \mathbf{F}_{1 k}^{\left(2^{l} m\right)}\left(\dot{\mathbf{a}}_{0}^{\left(2^{l} m\right)}, \dot{\mathbf{b}}^{\left(2^{l} m\right)}, \dot{\mathbf{c}}^{\left(2^{l} m\right)}, \mathbf{a}_{0}^{\left(2^{l} m\right)}, \mathbf{b}^{\left(2^{l} m\right)}, \mathbf{c}^{\left(2^{l} m\right)}\right), \\
& \ddot{\mathbf{c}}^{\left(2^{l} m\right)}-2 \frac{\Omega}{2^{l} m} \mathbf{k}_{1} \dot{\mathbf{b}}^{\left(2^{l} m\right)}-\frac{\Omega^{2}}{\left(2^{l} m\right)^{2}} \mathbf{k}_{2} \mathbf{c}^{\left(2^{l} m\right)} \\
= & \mathbf{F}_{2 k}^{\left(2^{l} m\right)}\left(\dot{\mathbf{a}}_{0}^{\left(2^{l} m\right)}, \dot{\mathbf{b}}^{\left(2^{l} m\right)}, \dot{\mathbf{c}}^{\left(2^{l} m\right)}, \mathbf{a}_{0}^{\left(2^{l} m\right)}, \mathbf{b}^{\left(2^{l} m\right)}, \mathbf{c}^{\left(2^{l} m\right)}\right) ;
\end{aligned}
$$

and for $N=1,2, \ldots, \infty$

$$
\begin{aligned}
& \mathbf{k}_{1}=\operatorname{diag}\left(\mathbf{I}_{n \times n}, 2 \mathbf{I}_{n \times n}, \ldots, N \mathbf{I}_{n \times n}\right), \\
& \mathbf{k}_{2}=\operatorname{diag}\left(\mathbf{I}_{n \times n}, 2^{2} \mathbf{I}_{n \times n}, \ldots, N^{2} \mathbf{I}_{n \times n}\right), \\
& \mathbf{b}^{\left(2^{l} m\right)}=\left(\mathbf{b}_{1 / 2^{l} m}, \mathbf{b}_{2 / 2^{l} m}, \ldots, \mathbf{b}_{N / 2^{l} m}\right)^{\mathrm{T}}, \\
& \mathbf{c}^{\left(2^{l} m\right)}=\left(\mathbf{c}_{1 / 2^{l} m}, \mathbf{c}_{2 / 2^{l} m}, \ldots, \mathbf{c}_{N / 2^{l} m}\right)^{\mathrm{T}}, \\
& \mathbf{F}_{1}^{\left(2^{l} m\right)}=\left(\mathbf{F}_{11}^{\left(2^{l} m\right)}, \mathbf{F}_{12}^{\left(2^{l} m\right)}, \ldots, \mathbf{F}_{1 N}^{\left(2^{l} m\right)}\right)^{\mathrm{T}}, \\
& \mathbf{F}_{2}^{\left(2^{l} m\right)}=\left(\mathbf{F}_{21}^{\left(2^{l} m\right)}, \mathbf{F}_{22}^{\left(2^{l} m\right)}, \ldots, \mathbf{F}_{2 N}^{\left(2^{l} m\right)}\right)^{\mathrm{T}} ; \\
& \mathbf{F}_{0}^{\left(2^{l} m\right)}\left(\mathbf{a}_{0}^{\left(2^{l} m\right)}, \mathbf{b}^{\left(2^{l} m\right)}, \mathbf{c}^{\left(2^{l} m\right)}, \dot{\mathbf{a}}_{0}^{\left(2^{l} m\right)}, \dot{\mathbf{b}}^{\left(2^{l} m\right)}, \dot{\mathbf{c}}^{\left(2^{l} m\right)}\right) \\
& =\frac{1}{2^{l} m T} \int_{0}^{2^{l} m T} \mathbf{f}\left(\mathbf{x}^{\left(2^{l} m\right) *}, \dot{\mathbf{x}}^{\left(2^{l} m\right) *}, \mathbf{p}\right) d t ; \\
& \mathbf{F}_{1 k}^{\left(2^{l} m\right)}\left(\mathbf{a}_{0}^{\left(2^{l} m\right)}, \mathbf{b}^{\left(2^{l} m\right)}, \mathbf{c}^{\left(2^{l} m\right)}, \dot{\mathbf{a}}_{0}^{\left(2^{l} m\right)}, \dot{\mathbf{b}}^{\left(2^{l} m\right)}, \dot{\mathbf{c}}^{\left(2^{l} m\right)}\right) \\
& =\frac{1}{2^{l-1} m T} \int_{0}^{2^{l} m T} \mathbf{f}\left(\mathbf{x}^{\left(2^{l} m\right) *}, \dot{\mathbf{x}}^{\left(2^{l} m\right) *}, \mathbf{p}\right) \cos \left(\frac{k}{2^{l} m} \Omega t\right) d t, \\
& \mathbf{F}_{2 k}^{\left(2^{l} m\right)}\left(\mathbf{a}_{0}^{\left(2^{l} m\right)}, \mathbf{b}^{\left(2^{l} m\right)}, \mathbf{c}^{\left(2^{l} m\right)}, \dot{\mathbf{a}}_{0}^{\left(2^{l} m\right)}, \dot{\mathbf{b}}^{\left(2^{l} m\right)}, \dot{\mathbf{c}}^{\left(2^{l} m\right)}\right) \\
& =\frac{1}{2^{l-1} m T} \int_{0}^{2^{l} m T} \mathbf{f}\left(\mathbf{x}^{\left(2^{l} m\right) *}, \dot{\mathbf{x}}^{\left(2^{l} m\right) *}, \mathbf{p}\right) \sin \left(\frac{k}{2^{l} m} \Omega t\right) d t .
\end{aligned}
$$

The solution of period- $2^{l} m$ motion can be determined by equilibriums of coefficient dynamical system in Eq. (78), and the corresponding stability and bifurcation can be done. As $l \rightarrow \infty$, the stable and unstable chaos with $\left(n_{1}, n_{2}, n_{3} \mid n_{4}, n_{5}, n_{6}\right)$ in Eq. (72) can be obtained where

$\sum_{i=1}^{3} n_{i}+2 \sum_{i=4}^{6} n_{i}=2 n(1+2 N)$

With increasing $l$, the Fourier truncated number $N>>2^{l} m$ will dramatically increase. If period- 1 motion possesses at least $N_{1}$ harmonic vector terms, then the total harmonic vector terms for period- $2^{l} m$ motion should be $N \geq 2^{l} m N_{1}$. The classification of chaotic motions with specific cases is given as follows.

(i) For the chaotic motion of $\left(n_{1}, 0,0 \mid 0,0,0\right)$ with $n_{1}=$ $2 n(1+2 N)$, the chaotic motion is called the hyperbolic stable chaos.

(ii) For the chaotic motion of $\left(0,0,0 \mid n_{4}, 0,0\right)$ with $n_{4}=$ $n(1+2 N)$, the chaotic motion is called the spiral stable chaos.

(iii) For the chaotic motion of $\left(n_{1}, 0,0 \mid n_{4}, 0,0\right)$ with $n_{1}+$ $2 n_{4}=2 n(1+2 N)$, the chaotic motion is called the hyperbolic-spiral stable chaos. 
(iv) For the chaotic motion of $\left(0, n_{2}, 0 \mid 0,0,0\right)$ with $n_{2}=$ $2 n(1+2 N)$, the chaotic motion is called the hyperbolic unstable chaos.

(v) For the chaotic motion of $\left(0,0,0 \mid 0, n_{5}, 0\right)$ with $n_{5}=$ $n(1+2 N)$, the chaotic motion is called the spiral unstable chaos.

(vi) For the chaotic motion of $\left(0, n_{2}, 0 \mid 0, n_{5}, 0\right)$ with $n_{2}+$ $2 n_{5}=2 n(1+2 N)$, the chaotic motion is called the hyperbolic-spiral unstable chaos.

(vii) For the chaotic motion of $\left(n_{1}, n_{2}, 0 \mid 0,0,0\right)$ with $n_{1}+$ $n_{2}=2 n(1+2 N)$, the chaotic motion is called the saddle unstable chaos.

(viii) For the chaotic motion of $\left(n_{1}, n_{2}, 0 \mid n_{4}, n_{5}, 0\right)$ with $n_{1}+n_{2}+2 n_{4}+2 n_{5}=2 n(1+2 N)$, the chaotic motion is called the spiral saddle unstable chaos.

Because $m$ is an arbitrary positive integer number, it includes $\left(2^{l} m_{1}\right)$ for period- $2^{l} m_{1}$ motion. Thus, the expression for period- $m$ motion can be used for any periodic motions. The expression in Eq. (61) can be used to express the solution for chaotic motion as $m \rightarrow \infty$. The solutions of chaotic motion can be classified as discussed for period- $2^{l} m_{1}$ motion.

\subsection{Periodically forced vibration systems}

Suppose such forced vibration systems possess periodic motions with period $T=2 \pi / \Omega$, then such periodic motions can be expressed by the Fourier series, discussed as follows.

Theorem 7 Consideraperiodically forced, nonlinear vibration system as

$\ddot{\mathbf{x}}=\mathbf{F}(\mathbf{x}, \dot{\mathbf{x}}, t, \mathbf{p}) \in \mathbf{R}^{n}$

where $\mathbf{F}(\mathbf{x}, \dot{\mathbf{x}}, t, \mathbf{p})$ is a $C^{r}$-continuous nonlinear function vector $(r \geq 1)$ with forcing period $T=2 \pi / \Omega$. If there is a periodic motion $\mathbf{x}^{*}(t)$ with finite norm $\left\|\mathbf{x}^{*}\right\|$, an analytical expression for the periodic motion of Eq. (82) is in the form of

$\mathbf{x}^{*}(t)=\mathbf{a}_{0}(t)+\sum_{k=1}^{N} \mathbf{b}_{k}(t) \cos (k \Omega t)+\mathbf{c}_{k}(t) \sin (k \Omega t)$.

with

$\mathbf{a}_{0}=\left(a_{01}, a_{02}, \ldots, a_{0 n}\right)^{\mathrm{T}}$,

$\mathbf{b}_{k}=\left(b_{k 1}, b_{k 2}, \ldots, b_{k n}\right)^{\mathrm{T}}$,

$\mathbf{c}_{k}=\left(c_{k 1}, c_{k 2}, \ldots, c_{k n}\right)^{\mathrm{T}}$

and the corresponding coefficients slowly varying with time are determined by

$\ddot{\mathbf{a}}_{0}=\mathbf{F}_{0}\left(\mathbf{a}_{0}, \mathbf{b}, \mathbf{c}, \dot{\mathbf{a}}_{0}, \dot{\mathbf{b}}, \dot{\mathbf{c}}\right)$,

$\ddot{\mathbf{b}}=-2 \Omega \mathbf{k}_{1} \dot{\mathbf{c}}+\Omega^{2} \mathbf{k}_{2} \mathbf{b}+\mathbf{F}_{1}\left(\mathbf{a}_{0}, \mathbf{b}, \mathbf{c}, \dot{\mathbf{a}}_{0}, \dot{\mathbf{b}}, \dot{\mathbf{c}}\right)$,

$\ddot{\mathbf{c}}=2 \Omega \mathbf{k}_{1} \dot{\mathbf{b}}+\Omega^{2} \mathbf{k}_{2} \mathbf{c}+\mathbf{F}_{2}\left(\mathbf{a}_{0}, \mathbf{b}, \mathbf{c}, \dot{\mathbf{a}}_{0}, \dot{\mathbf{b}}, \dot{\mathbf{c}}\right) ;$ where for $N=1,2, \ldots, \infty$.

$\mathbf{k}_{1}=\operatorname{diag}\left(\mathbf{I}_{n \times n}, 2 \mathbf{I}_{n \times n}, \ldots, N \mathbf{I}_{n \times n}\right)$,

$\mathbf{k}_{2}=\operatorname{diag}\left(\mathbf{I}_{n \times n}, 2^{2} \mathbf{I}_{n \times n}, \ldots, N^{2} \mathbf{I}_{n \times n}\right)$,

$\mathbf{b}=\left(\mathbf{b}_{1}, \mathbf{b}_{2}, \ldots, \mathbf{b}_{N}\right)^{\mathrm{T}}$ and $\mathbf{c}=\left(\mathbf{c}_{1}, \mathbf{c}_{2}, \ldots, \mathbf{c}_{N}\right)^{\mathrm{T}}$,

$\mathbf{F}_{1}=\left(\mathbf{F}_{11}, \mathbf{F}_{12}, \ldots, \mathbf{F}_{1 N}\right)^{\mathrm{T}}$ and $\mathbf{F}_{2}=\left(\mathbf{F}_{21}, \mathbf{F}_{22}, \ldots, \mathbf{F}_{2 N}\right)^{\mathrm{T}}$.

and for $k=1,2, \ldots, N$

$\mathbf{F}_{0}\left(\mathbf{a}_{0}, \mathbf{b}, \mathbf{c}, \dot{\mathbf{a}}_{0}, \dot{\mathbf{b}}, \dot{\mathbf{c}}\right)=\frac{1}{T} \int_{0}^{T} \mathbf{F}\left(\mathbf{x}^{*}, \dot{\mathbf{x}}^{*}, t, \mathbf{p}\right) d t ;$

$\mathbf{F}_{1 k}\left(\mathbf{a}_{0}, \mathbf{b}, \mathbf{c}, \dot{\mathbf{a}}_{0}, \dot{\mathbf{b}}, \dot{\mathbf{c}}\right)=\frac{2}{T} \int_{0}^{T} \mathbf{F}\left(\mathbf{x}^{*}, \dot{\mathbf{x}}^{*}, t, \mathbf{p}\right) \cos (k \Omega t) d t$,

$\mathbf{F}_{2 k}\left(\mathbf{a}_{0}, \mathbf{b}, \mathbf{c}, \dot{\mathbf{a}}_{0}, \dot{\mathbf{b}}, \dot{\mathbf{c}}\right)=\frac{2}{T} \int_{0}^{T} \mathbf{F}\left(\mathbf{x}^{*}, \dot{\mathbf{x}}^{*}, t, \mathbf{p}\right) \sin (k \Omega t) d t$.

The state-space form of Eq. (85) is

$\dot{\mathbf{z}}=\mathbf{z}_{1} \quad$ and $\quad \dot{\mathbf{z}}_{1}=\mathbf{g}\left(\mathbf{z}, \mathbf{z}_{1}\right)$

where

$\mathbf{z}=\left(\mathbf{a}_{0}, \mathbf{b}, \mathbf{c}\right)^{\mathrm{T}}, \quad \dot{\mathbf{z}}=\mathbf{z}_{1}$

$\mathbf{g}=\left(\mathbf{F}_{0},-2 \Omega \mathbf{k}_{1} \dot{\mathbf{c}}+\Omega^{2} \mathbf{k}_{2} \mathbf{b}+\mathbf{F}_{1}, 2 \Omega \mathbf{k}_{1} \dot{\mathbf{b}}+\Omega^{2} \mathbf{k}_{2} \mathbf{c}+\mathbf{F}_{2}\right)^{\mathrm{T}}$.

An equivalent system of Eq. (88) is

$\dot{\mathbf{y}}=\mathbf{f}(\mathbf{y})$

where

$\mathbf{y}=\left(\mathbf{z}, \mathbf{z}_{1}\right)^{\mathrm{T}}$ and $\mathbf{f}=\left(\mathbf{z}_{1}, \mathbf{g}\right)^{\mathrm{T}}$

If equilibrium $\mathbf{y}^{*}$ of Eq. (90) (i.e., $\mathbf{f}\left(\mathbf{y}^{*}\right)=\mathbf{0}$ ) exists, then the analytical solution of the periodic motion exists in Eq. (83). In vicinity of equilibrium $\mathbf{y}^{*}$, with $\mathbf{y}=\mathbf{y}^{*}+\Delta \mathbf{y}$ the linearized equation of Eq. (90) is

$\Delta \dot{\mathbf{y}}=D \mathbf{f}\left(\mathbf{y}^{*}\right) \Delta \mathbf{y}$

and the eigenvalue analysis of equilibrium $\mathbf{y}^{*}$ is given by

$\left|D \mathbf{f}\left(\mathbf{y}^{*}\right)-\lambda \mathbf{I}_{2 n(2 N+1) \times 2 n(2 N+1)}\right|=0$

where Dff( $\left.\mathbf{y}^{*}\right)=\partial \mathbf{f}(\mathbf{y}) /\left.\partial \mathbf{y}\right|_{\mathbf{y}^{*}}$. Thus, the stability and bifurcation of periodic motion can be classified by the eigenvalues of $D \mathbf{f}\left(\mathbf{y}^{*}\right)$ with

$\left(n_{1}, n_{2}, n_{3} \mid n_{4}, n_{5}, n_{6}\right)$

(i) If all eigenvalues of the equilibrium possess negative real parts, the approximate periodic solution is stable. 
(ii) If at least one of eigenvalues of the equilibrium possesses positive real part, the approximate periodic solution is unstable.

(iii) The boundaries between stable and unstable equilibriums with higher order singularity give bifurcation and stability conditions with higher order singularity.

Proof The proof can be referred to Luo [39].

Similarly, the periodic- $m$ motion in periodically forced, nonlinear vibration system will be discussed.

Theorem 8 Consider a periodically forced, nonlinear vibration system in Eq. (82) with a forcing period $T=2 \pi / \Omega$. If there is a period-m motion $\mathbf{x}^{(m) *}(t)$ with finite norm $\left\|\mathbf{x}^{(m) *}\right\|$, an analytical expression for the period-m motion of Eq. (82) is

$$
\begin{aligned}
\mathbf{x}^{(m) *}(t)= & \mathbf{a}_{0}^{(m)}(t)+\sum_{k=1}^{N} \mathbf{b}_{k / m}(t) \cos \left(\frac{k}{m} \Omega t\right) \\
& +\mathbf{c}_{k / m}(t) \sin \left(\frac{k}{m} \Omega t\right) .
\end{aligned}
$$

with for $k=1,2, \ldots, N$

$$
\begin{aligned}
& \mathbf{a}_{0}^{(m)}=\left(a_{01}^{(m)}, a_{02}^{(m)}, \ldots, a_{0 n}^{(m)}\right)^{\mathrm{T}}, \\
& \mathbf{b}_{k / m}=\left(b_{k / m 1}, b_{k / m 2}, \ldots, b_{k / m n}\right)^{\mathrm{T}}, \\
& \mathbf{c}_{k / m}=\left(c_{k / m 1}, c_{k / m 2}, \ldots, c_{k / m n}\right)^{\mathrm{T}}
\end{aligned}
$$

and the corresponding coefficients slowly varying with time are determined by

$$
\begin{aligned}
\ddot{\mathbf{a}}_{0}^{(m)}= & \mathbf{F}_{0}^{(m)}\left(\mathbf{a}_{0}^{(m)}, \mathbf{b}^{(m)}, \mathbf{c}^{(m)}, \dot{\mathbf{a}}_{0}^{(m)}, \dot{\mathbf{b}}^{(m)}, \dot{\mathbf{c}}^{(m)}\right), \\
\ddot{\mathbf{b}}^{(m)}= & -2 \frac{\Omega}{m} \mathbf{k}_{1} \dot{\mathbf{c}}^{(m)}+\frac{\Omega^{2}}{m^{2}} \mathbf{k}_{2} \mathbf{b}^{(m)} \\
& +\mathbf{F}_{1}^{(m)}\left(\mathbf{a}_{0}^{(m)}, \mathbf{b}^{(m)}, \mathbf{c}^{(m)}, \dot{\mathbf{a}}_{0}^{(m)}, \dot{\mathbf{b}}^{(m)}, \dot{\mathbf{c}}^{(m)}\right), \\
\ddot{\mathbf{c}}^{(m)}= & 2 \frac{\Omega}{m} \mathbf{k}_{1} \dot{\mathbf{b}}^{(m)}+\frac{\Omega^{2}}{m^{2}} \mathbf{k}_{2} \mathbf{c}^{(m)} \\
& +\mathbf{F}_{2}^{(m)}\left(\mathbf{a}_{0}^{(m)}, \mathbf{b}^{(m)}, \mathbf{c}^{(m)}, \dot{\mathbf{a}}_{0}^{(m)}, \dot{\mathbf{b}}^{(m)}, \dot{\mathbf{c}}^{(m)}\right)
\end{aligned}
$$

where for $N=1,2, \ldots, \infty$.

$$
\begin{aligned}
& \mathbf{k}_{1}=\operatorname{diag}\left(\mathbf{I}_{n \times n}, 2 \mathbf{I}_{n \times n}, \ldots, N \mathbf{I}_{n \times n}\right), \\
& \mathbf{k}_{2}=\operatorname{diag}\left(\mathbf{I}_{n \times n}, 2^{2} \mathbf{I}_{n \times n}, \ldots, N^{2} \mathbf{I}_{n \times n}\right), \\
& \mathbf{b}^{(m)}=\left(\mathbf{b}_{1 / m}, \mathbf{b}_{2 / m}, \ldots, \mathbf{b}_{N / m}\right)^{\mathrm{T}} \\
& \mathbf{c}^{(m)}=\left(\mathbf{c}_{1 / m}, \mathbf{c}_{2 / m}, \ldots, \mathbf{c}_{N / m}\right)^{\mathrm{T}} \\
& \mathbf{F}_{1}^{(m)}=\left(\mathbf{F}_{11}^{(m)}, \mathbf{F}_{12}^{(m)}, \ldots, \mathbf{F}_{1 N}^{(m)}\right)^{\mathrm{T}} \\
& \mathbf{F}_{2}^{(m)}=\left(\mathbf{F}_{21}^{(m)}, \mathbf{F}_{22}^{(m)}, \ldots, \mathbf{F}_{2 N}^{(m)}\right)^{\mathrm{T}}
\end{aligned}
$$

and for $k=1,2, \ldots, N$

$$
\begin{aligned}
& \mathbf{F}_{0}^{(m)}\left(\mathbf{a}_{0}^{(m)}, \mathbf{b}^{(m)}, \mathbf{c}^{(m)}, \dot{\mathbf{a}}^{(m)}, \dot{\mathbf{b}}^{(m)}, \dot{\mathbf{c}}^{(m)}\right) \\
& =\frac{1}{m T} \int_{0}^{m T} \mathbf{F}\left(\mathbf{x}^{(m) *}, \dot{\mathbf{x}}^{(m) *}, t, \mathbf{p}\right) d t ; \\
& \mathbf{F}_{1 k}^{(m)}\left(\mathbf{a}_{0}^{(m)}, \mathbf{b}^{(m)}, \mathbf{c}^{(m)}, \dot{\mathbf{a}}_{0}^{(m)}, \dot{\mathbf{b}}^{(m)}, \dot{\mathbf{c}}^{(m)}\right) \\
& =\frac{2}{m T} \int_{0}^{m T} \mathbf{F}\left(\mathbf{x}^{(m) *}, \dot{\mathbf{x}}^{(m) *}, t, \mathbf{p}\right) \cos \left(\frac{k}{m} \Omega t\right) d t, \\
& \mathbf{F}_{2 k}^{(m)}\left(\mathbf{a}_{0}^{(m)}, \mathbf{b}^{(m)}, \mathbf{c}^{(m)}, \dot{\mathbf{a}}_{0}^{(m)}, \dot{\mathbf{b}}^{(m)}, \dot{\mathbf{c}}^{(m)}\right) \\
& =\frac{2}{m T} \int_{0}^{m T} \mathbf{F}\left(\mathbf{x}^{(m) *}, \dot{\mathbf{x}}^{(m) *}, t, \mathbf{p}\right) \sin \left(\frac{k}{m} \Omega t\right) d t .
\end{aligned}
$$

The state-space form of Eq. (97) is

$\dot{\mathbf{z}}^{(m)}=\mathbf{z}_{1}^{(m)}$ and $\dot{\mathbf{z}}_{1}^{(m)}=\mathbf{g}^{(m)}\left(\mathbf{z}^{(m)}, \mathbf{z}_{1}^{(m)}\right)$

where

$$
\begin{aligned}
\mathbf{z}^{(m)}= & \left(\mathbf{a}_{0}^{(m)}, \mathbf{b}^{(m)}, \mathbf{c}^{(m)}\right)^{\mathrm{T}}, \dot{\mathbf{z}}^{(m)}=\mathbf{z}_{1}^{(m)} \\
\mathbf{g}^{(m)}= & \left(\mathbf{F}_{0}^{(m)},-2 \frac{\Omega}{m} \mathbf{k}_{1} \dot{\mathbf{c}}^{(m)}+\frac{\Omega^{2}}{m^{2}} \mathbf{k}_{2} \mathbf{b}^{(m)}+\mathbf{F}_{1}^{(m)},\right. \\
& \left.2 \frac{\Omega}{m} \mathbf{k}_{1} \dot{\mathbf{b}}^{(m)}+\frac{\Omega^{2}}{m^{2}} \mathbf{k}_{2} \mathbf{c}^{(m)}+\mathbf{F}_{2}^{(m)}\right)^{\mathrm{T}} .
\end{aligned}
$$

An equivalent system of Eq. (100) is

$\dot{\mathbf{y}}^{(m)}=\mathbf{f}^{(m)}\left(\mathbf{y}^{(m)}\right)$

where

$\mathbf{y}^{(m)}=\left(\mathbf{z}^{(m)}, \mathbf{z}_{1}^{(m)}\right)^{\mathrm{T}}$ and $\mathbf{f}^{(m)}=\left(\mathbf{z}_{1}^{(m)}, \mathbf{g}^{(m)}\right)^{\mathrm{T}}$.

If equilibrium $\mathbf{y}^{(m) *}$ of Eq. (102) (i.e., $\left.\mathbf{f}^{(m)}\left(\mathbf{y}^{(m) *}\right)=\mathbf{0}\right)$ exists, then the approximate analytical solution of the period-m motion exists in Eq. (95). In vicinity of equilibrium $\mathbf{y}^{(m) *}$, with $\mathbf{y}^{(m)}=\mathbf{y}^{(m) *}+\Delta \mathbf{y}^{(m)}$, the linearized equation of $E q$. (102) is

$\Delta \dot{\mathbf{y}}^{(m)}=D \mathbf{f}^{(m)}\left(\mathbf{y}^{(m) *}\right) \Delta \mathbf{y}^{(m)}$

and the eigenvalue analysis of equilibrium $\mathbf{y}^{*}$ is given by

$$
\left|D \mathbf{f}^{(m)}\left(\mathbf{y}^{(m) *}\right)-\lambda \mathbf{I}_{2 n(2 N+1) \times 2 n(2 N+1)}\right|=0
$$

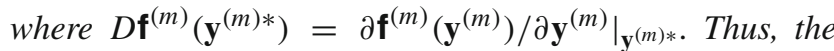
stability and bifurcation of periodic motion can be classified by the eigenvalues of $D \mathbf{f}^{(m)}\left(\mathbf{y}^{(m) *}\right)$ with

$\left(n_{1}, n_{2}, n_{3} \mid n_{4}, n_{5}, n_{6}\right)$

(i) If all eigenvalues of the equilibrium possess negative real parts, the approximate periodic solution is stable.

(ii) If at least one of eigenvalues of the equilibrium possesses positive real part, the approximate periodic solution is unstable. 
(iii) The boundaries between stable and unstable equilibriums with higher order singularity give bifurcation and stability conditions with higher order singularity.

Proof The proof can be referred to Luo [39].

\section{Autonomous time-delayed nonlinear systems}

In this section, the analytical periodic flows in autonomous, time-delayed, nonlinear dynamical systems will be discussed, and the local stability and bifurcation theory of equilibriums in a time-delayed, nonlinear system of coefficients will be used to classify analytical solutions of periodic flows and chaos in time-delayed, nonlinear dynamical systems. A generalized harmonic balance method will be presented for periodic flows and chaos in time-delayed, nonlinear dynamical systems.

\subsection{Autonomous, time-delayed, nonlinear systems}

Periodic flows in autonomous, time-delayed, dynamical systems will be presented herein. If such a system has a periodic flow with a period of $T=2 \pi / \Omega$, then such a periodic flow can be expressed by the Fourier series.

Theorem 9 Consider a nonlinear, time-delayed dynamical system as

$\dot{\mathbf{x}}=\mathbf{f}\left(\mathbf{x}, \mathbf{x}^{\tau}, \mathbf{p}\right) \in \mathbf{R}^{n}$

where $\mathbf{f}\left(\mathbf{x}, \mathbf{x}^{\tau}, \mathbf{p}\right)$ is a $C^{r}$-continuous nonlinear function vector $(r \geq 1)$. If such a dynamical system has a periodic flow $\mathbf{x}^{*}(t)$ with finite norm $\left\|\mathbf{x}^{*}\right\|$ and period $T=2 \pi / \Omega$, there is an analytical expression for the periodic flow of Eq. (107) in the form of

$$
\begin{aligned}
\mathbf{x}^{*} \equiv & \mathbf{x}^{*}(t)=\mathbf{a}_{0}(t)+\sum_{k=1}^{N} \mathbf{b}_{k}(t) \cos (k \Omega t)+\mathbf{c}_{k}(t) \sin (k \Omega t) \\
\mathbf{x}^{\tau *} \equiv & \mathbf{x}^{*}(t-\tau)=\mathbf{a}_{0}^{\tau}(t) \\
& +\sum_{k=1}^{N} \mathbf{b}_{k}^{\tau}(t) \cos [k \Omega(t-\tau)] \\
& +\mathbf{c}_{k}^{\tau}(t) \sin [k \Omega(t-\tau)]
\end{aligned}
$$

with $\mathbf{a}_{0}^{\tau}=\mathbf{a}_{0}(t-\tau), \mathbf{b}_{k}^{\tau}=\mathbf{b}_{k}(t-\tau), \mathbf{c}_{k}^{\tau}=\mathbf{c}_{k}(t-\tau)$ and

$\mathbf{a}_{0}=\left(a_{01}, a_{02}, \ldots, a_{0 n}\right)^{\mathrm{T}}$,

$\mathbf{b}_{k}=\left(b_{k 1}, b_{k 2}, \ldots, b_{k n}\right)^{\mathrm{T}}$,

$\mathbf{c}_{k}=\left(c_{k 1}, c_{k 2}, \ldots, c_{k n}\right)^{\mathrm{T}}$;

$\mathbf{a}_{0}^{\tau}=\left(a_{01}^{\tau}, a_{02}^{\tau}, \ldots, a_{0 n}^{\tau}\right)^{\mathrm{T}}$,

$\mathbf{b}_{k}^{\tau}=\left(b_{k 1}^{\tau}, b_{k 2}^{\tau}, \ldots, b_{k n}^{\tau}\right)^{\mathrm{T}}$,

$\mathbf{c}_{k}^{\tau}=\left(c_{k 1}^{\tau}, c_{k 2}^{\tau}, \ldots, c_{k n}^{\tau}\right)^{\mathrm{T}}$; and the corresponding coefficients slowly varying with time are determined by

$\dot{\mathbf{a}}_{0}=\mathbf{F}_{0}\left(\mathbf{z}, \mathbf{z}^{\tau}\right)$,

$\dot{\mathbf{b}}=-\Omega \mathbf{k}_{1} \mathbf{c}+\mathbf{F}_{1}\left(\mathbf{z}, \mathbf{z}^{\tau}\right)$,

$\dot{\mathbf{c}}=\Omega \mathbf{k}_{1} \mathbf{b}+\mathbf{F}_{2}\left(\mathbf{z}, \mathbf{z}^{\tau}\right)$;

where

$\mathbf{k}_{1}=\operatorname{diag}\left(\mathbf{I}_{n \times n}, 2 \mathbf{I}_{n \times n}, \ldots, N \mathbf{I}_{n \times n}\right)$,

$\mathbf{b}=\left(\mathbf{b}_{1}, \mathbf{b}_{2}, \ldots, \mathbf{b}_{N}\right)^{\mathrm{T}}$,

$\mathbf{c}=\left(\mathbf{c}_{1}, \mathbf{c}_{2}, \ldots, \mathbf{c}_{N}\right)^{\mathrm{T}}$;

$\mathbf{b}^{\tau}=\left(\mathbf{b}_{1}^{\tau}, \mathbf{b}_{2}^{\tau}, \ldots, \mathbf{b}_{N}^{\tau}\right)^{\mathrm{T}}$,

$\mathbf{c}^{\tau}=\left(\mathbf{c}_{1}^{\tau}, \mathbf{c}_{2}^{\tau}, \ldots, \mathbf{c}_{N}^{\tau}\right)^{\mathrm{T}}$;

$\mathbf{F}_{1}=\left(\mathbf{F}_{11}, \mathbf{F}_{12}, \ldots, \mathbf{F}_{1 N}\right)^{\mathrm{T}}$,

$\mathbf{F}_{2}=\left(\mathbf{F}_{21}, \mathbf{F}_{22}, \ldots, \mathbf{F}_{2 N}\right)^{\mathrm{T}}$;

$\mathbf{z}=\left(\mathbf{a}_{0}, \mathbf{b}, \mathbf{c}\right)^{\mathrm{T}}$,

$\mathbf{z}^{\tau}=\left(\mathbf{a}_{0}^{\tau}, \mathbf{b}^{\tau}, \mathbf{c}^{\tau}\right)^{\mathrm{T}}$

for $N=1,2, \ldots, \infty$.

and

$\mathbf{F}_{0}\left(\mathbf{z}, \mathbf{z}^{\tau}\right)=\frac{1}{T} \int_{0}^{T} \mathbf{f}\left(\mathbf{x}^{*}, \mathbf{x}^{\tau *}, \mathbf{p}\right) d t$

$\mathbf{F}_{1 k}\left(\mathbf{z}, \mathbf{z}^{\tau}\right)=\frac{2}{T} \int_{0}^{T} \mathbf{f}\left(\mathbf{x}^{*}, \mathbf{x}^{\tau *}, \mathbf{p}\right) \cos (k \Omega t) d t$,

$\mathbf{F}_{2 k}\left(\mathbf{z}, \mathbf{z}^{\tau}\right)=\frac{2}{T} \int_{0}^{T} \mathbf{f}\left(\mathbf{x}^{*}, \mathbf{x}^{\tau *}, \mathbf{p}\right) \sin (k \Omega t) d t ;$

for $k=1,2, \ldots, N$.

Equation (110) becomes

$\dot{\mathbf{z}}=\mathbf{f}\left(\mathbf{z}, \mathbf{z}^{\tau}\right)$

where

$\mathbf{f}=\left(\mathbf{F}_{0},-\Omega \mathbf{k}_{1} \mathbf{c}+\mathbf{F}_{1}, \Omega \mathbf{k}_{1} \mathbf{b}+\mathbf{F}_{2}\right)^{\mathrm{T}}$

If equilibrium $\left(\mathbf{z}^{*}=\mathbf{z}^{\tau *}\right)$ of Eq. (113) (i.e., $\mathbf{f}\left(\mathbf{z}^{*}, \mathbf{z}^{* \tau}\right)=\mathbf{0}$ ) exists, then the analytical solution of periodic flow of Eq. (107) exists in Eq. (108). In vicinity of equilibrium $\mathbf{z}^{*}=\mathbf{z}^{\tau *}$, with $\mathbf{z}=\mathbf{z}^{*}+\Delta \mathbf{z}$ and $\mathbf{z}^{\tau}=\mathbf{z}^{\tau *}+\Delta \mathbf{z}^{\tau}$ the linearized equation of Eq. (113) is

$\Delta \dot{\mathbf{z}}=D_{\mathbf{z}} \mathbf{f}\left(\mathbf{z}^{*}, \mathbf{z}^{* \tau}\right) \Delta \mathbf{z}+D_{\mathbf{z}^{\tau}} \mathbf{f}\left(\mathbf{z}^{*}, \mathbf{z}^{* \tau}\right) \Delta \mathbf{z}^{\tau}$

and the eigenvalue analysis of equilibrium $\mathbf{z}^{*}=\mathbf{z}^{\tau *}$ is given by

$$
\begin{aligned}
& \mid D_{\mathbf{z}} \mathbf{f}\left(\mathbf{z}^{*}, \mathbf{z}^{\tau *}\right)-\lambda \mathbf{I}_{n(2 N+1) \times n(2 N+1)} \\
& \quad+D_{\mathbf{z}} \mathbf{f}\left(\mathbf{z}^{*}, \mathbf{z}^{\tau *}\right) e^{-\lambda \tau} \mid=0
\end{aligned}
$$


where

$$
\begin{aligned}
& D_{\mathbf{z}} \mathbf{f}\left(\mathbf{z}^{*}, \mathbf{z}^{\tau *}\right)=\partial \mathbf{f}\left(\mathbf{z}, \mathbf{z}^{\tau}\right) /\left.\partial \mathbf{z}\right|_{\left(\mathbf{z}^{*}, \mathbf{z}^{\tau *}\right)}, \\
& D_{\mathbf{z}^{\tau}} \mathbf{f}\left(\mathbf{z}^{*}, \mathbf{z}^{\tau *}\right)=\partial \mathbf{f}\left(\mathbf{z}, \mathbf{z}^{\tau}\right) /\left.\partial \mathbf{z}^{\tau}\right|_{\left(\mathbf{z}^{*}, \mathbf{z}^{\tau *}\right)} .
\end{aligned}
$$

Thus, the stability and bifurcation of periodic solution can be classified by the eigenvalues of Df $\left(\mathbf{z}^{*}\right)$ with

$\left(n_{1}, n_{2}, n_{3} \mid n_{4}, n_{5}, n_{6}\right)$.

(i) If all eigenvalues of the equilibrium possess negative real parts, the approximate periodic solution is stable.

(ii) If at least one of eigenvalues of the equilibrium possesses positive real part, the approximate periodic solution is unstable.

(iii) The boundaries between stable and unstable equilibriums with higher order singularity give bifurcation and stability conditions with higher order singularity.

Proof If $\mathbf{f}\left(\mathbf{x}, \mathbf{x}^{\tau}, \mathbf{p}\right)$ is a $C^{r}$-continuous nonlinear function vector $(r \geq 1)$, then the velocity $\dot{\mathbf{x}}$ should be $C^{r}$-continuous $(r \geq 1)$, and the acceleration $\ddot{\mathbf{x}}$ should be bounded (i.e., ||$\ddot{\mathbf{x}}|| \leq K$ ). Thus, the Fourier series expression of periodic flow in Eq. (108) is convergent from the Fourier series theory. Taking the derivative of Eq. (108) with respect to time gives

$$
\begin{aligned}
\dot{\mathbf{x}}^{*}(t)= & \dot{\mathbf{a}}_{0}+\sum_{k=1}^{N}\left[\dot{\mathbf{b}}_{k}+k \Omega \mathbf{c}_{k}\right] \cos (k \Omega t) \\
& +\left[\dot{\mathbf{c}}_{k}-k \Omega \mathbf{b}_{k}\right] \sin (k \Omega t) . \\
\dot{\mathbf{x}}^{\tau *}(t)= & \dot{\mathbf{a}}_{0}^{\tau}+\sum_{k=1}^{N}\left[\dot{\mathbf{b}}_{k}^{\tau}+k \Omega \mathbf{c}_{k}^{\tau}\right] \cos [k \Omega(t-\tau)] \\
& +\left[\dot{\mathbf{c}}_{k}^{\tau}-k \Omega \mathbf{b}_{k}^{\tau}\right] \sin [k \Omega(t-\tau)]
\end{aligned}
$$

where $\mathbf{a}_{0}^{\tau}=\mathbf{a}_{0}(t-\tau), \mathbf{b}^{\tau}=\mathbf{b}(t-\tau), \mathbf{c}^{\tau}=\mathbf{c}(t-\tau)$. Because the coefficients vary slowly with time, substitution of the foregoing equation into the time-delayed system in Eq. (107) and averaging for constant, $\cos (k \Omega t)$ and $\sin (k \Omega t)$ yields fork $=1,2, \ldots, N$

$$
\begin{aligned}
& \dot{\mathbf{a}}_{0}=\mathbf{F}_{0}\left(\mathbf{z}, \mathbf{z}^{\tau}\right), \\
& \dot{\mathbf{b}}_{k}=-\Omega k \mathbf{c}_{k}+\mathbf{F}_{1 k}\left(\mathbf{z}, \mathbf{z}^{\tau}\right), \\
& \dot{\mathbf{c}}_{k}=\Omega k \mathbf{b}_{k}+\mathbf{F}_{2 k}\left(\mathbf{z}, \mathbf{z}^{\tau}\right)
\end{aligned}
$$

where

$$
\begin{aligned}
& \mathbf{F}_{0}\left(\mathbf{z}, \mathbf{z}^{\tau}\right)=\frac{1}{T} \int_{0}^{T} \mathbf{f}\left(\mathbf{x}^{*}, \mathbf{x}^{\tau *}, \mathbf{p}\right) d t \\
& \mathbf{F}_{1 k}\left(\mathbf{z}, \mathbf{z}^{\tau}\right)=\frac{2}{T} \int_{0}^{T} \mathbf{f}\left(\mathbf{x}^{*}, \mathbf{x}^{\tau *}, \mathbf{p}\right) \cos (k \Omega t) d t,
\end{aligned}
$$

$\mathbf{F}_{2 k}\left(\mathbf{z}, \mathbf{z}^{\tau}\right)=\frac{2}{T} \int_{0}^{T} \mathbf{f}\left(\mathbf{x}^{*}, \mathbf{x}^{\tau *}, \mathbf{p}\right) \sin (k \Omega t) d t$

for $k=1,2, \ldots, N$;

and

$\mathbf{k}_{1}=\operatorname{diag}\left(\mathbf{I}_{n \times n}, 2 \mathbf{I}_{n \times n}, \ldots, N \mathbf{I}_{n \times n}\right)$,

$\mathbf{b}=\left(\mathbf{b}_{1}, \mathbf{b}_{2}, \ldots, \mathbf{b}_{N}\right)^{\mathrm{T}}$,

$\mathbf{c}=\left(\mathbf{c}_{1}, \mathbf{c}_{2}, \ldots, \mathbf{c}_{N}\right)^{\mathrm{T}}$

$\mathbf{b}^{\tau}=\left(\mathbf{b}_{1}^{\tau}, \mathbf{b}_{2}^{\tau}, \ldots, \mathbf{b}_{N}^{\tau}\right)^{\mathrm{T}}$,

$\mathbf{c}^{\tau}=\left(\mathbf{c}_{1}^{\tau}, \mathbf{c}_{2}^{\tau}, \ldots, \mathbf{c}_{N}^{\tau}\right)^{\mathrm{T}}$;

$\mathbf{F}_{1}=\left(\mathbf{F}_{11}, \mathbf{F}_{12}, \ldots, \mathbf{F}_{1 N}\right)^{\mathrm{T}}$,

$\mathbf{F}_{2}=\left(\mathbf{F}_{21}, \mathbf{F}_{22}, \ldots, \mathbf{F}_{2 N}\right)^{\mathrm{T}}$,

$\mathbf{z}=\left(\mathbf{a}_{0}, \mathbf{b}, \mathbf{c}\right)^{\mathrm{T}}$,

$\mathbf{z}^{\tau}=\left(\mathbf{a}_{0}^{\tau}, \mathbf{b}^{\tau}, \mathbf{c}^{\tau}\right)^{\mathrm{T}}$

for $N=1,2, \ldots, \infty$.

Rearranging the foregoing equation gives Eq. (110), i.e.,

$\dot{\mathbf{a}}_{0}=\mathbf{F}_{0}\left(\mathbf{z}, \mathbf{z}^{\tau}\right)$,

$\dot{\mathbf{b}}=-\Omega \mathbf{k}_{1} \mathbf{c}+\mathbf{F}_{1}\left(\mathbf{z}, \mathbf{z}^{\tau}\right)$,

$\dot{\mathbf{c}}=\Omega \mathbf{k}_{1} \mathbf{b}+\mathbf{F}_{2}\left(\mathbf{z}, \mathbf{z}^{\tau}\right)$.

Introduce

$\mathbf{f}=\left(\mathbf{F}_{0},-\Omega \mathbf{k}_{1} \mathbf{c}+\mathbf{F}_{1}, \Omega \mathbf{k}_{1} \mathbf{b}+\mathbf{F}_{2}\right)^{\mathrm{T}}$.

The equation in Eq. (110) becomes

$\dot{\mathbf{z}}=\mathbf{f}\left(\mathbf{z}, \mathbf{z}^{\tau}\right)$

Equilibriums of the foregoing equation is given by $\mathbf{f}\left(\mathbf{z}^{*}, \mathbf{z}^{\tau *}\right)$ $=\mathbf{0}$, i.e.,

$\mathbf{0}=\mathbf{F}_{0}\left(\mathbf{a}_{0}^{*}, \mathbf{b}^{*}, \mathbf{c}^{*}, \mathbf{a}_{0}^{\tau *}, \mathbf{b}^{\tau *}, \mathbf{c}^{\tau *}\right)$,

$\mathbf{0}=-\Omega \mathbf{k}_{1} \mathbf{c}^{*}+\mathbf{F}_{1}\left(\mathbf{a}_{0}^{*}, \mathbf{b}^{*}, \mathbf{c}^{*}, \mathbf{a}_{0}^{\tau *}, \mathbf{b}^{\tau *}, \mathbf{c}^{\tau *}\right)$,

$\mathbf{0}=\Omega \mathbf{k}_{1} \mathbf{b}^{*}+\mathbf{F}_{2}\left(\mathbf{a}_{0}^{*}, \mathbf{b}^{*}, \mathbf{c}^{*}, \mathbf{a}_{0}^{\tau *}, \mathbf{b}^{\tau *}, \mathbf{c}^{\tau *}\right)$

with

$\mathbf{a}_{0}^{*}=\mathbf{a}_{0}^{\tau *}, \mathbf{b}^{*}=\mathbf{b}^{\tau *}, \mathbf{c}^{*}=\mathbf{c}^{\tau *}$.

Thus, solutions of the forgoing equation are the existence conditions of periodic solutions for time-delayed, nonlinear dynamical systems. If the foregoing equation gives equilibrium $\mathbf{z}^{*}=\mathbf{z}^{\tau *}$, in the vicinity of $\mathbf{z}^{*}$ and $\mathbf{z}^{\tau *}$, with $\mathbf{z}=\mathbf{z}^{*}+\Delta \mathbf{z}$ and $\mathbf{z}^{\tau}=\mathbf{z}^{\tau *}+\Delta \mathbf{z}^{\tau}$ the linearized equation of $\dot{\mathbf{z}}=\mathbf{f}\left(\mathbf{z}, \mathbf{z}^{\tau}\right)$ is

$\Delta \dot{\mathbf{z}}=D_{\mathbf{z}} \mathbf{f}\left(\mathbf{z}^{*}, \mathbf{z}^{\tau *}\right) \Delta \mathbf{z}+D_{\mathbf{z}^{\tau}} \mathbf{f}\left(\mathbf{z}^{*}, \mathbf{z}^{\tau *}\right) \Delta \mathbf{z}^{\tau}$

and the eigenvalue analysis of equilibrium $\mathbf{z}^{*}=\mathbf{z}^{\tau *}$ is completed via

$$
\begin{aligned}
& \mid D_{\mathbf{z}} \mathbf{f}\left(\mathbf{z}^{*}, \mathbf{z}^{\tau *}\right)-\lambda \mathbf{I}_{n(2 N+1) \times n(2 N+1)} \\
& \quad+D_{\mathbf{z}^{\tau}} \mathbf{f}\left(\mathbf{z}^{*}, \mathbf{z}^{\tau *}\right) e^{-\lambda \tau} \mid=0
\end{aligned}
$$


where

$$
\begin{aligned}
& D_{\mathbf{z}} \mathbf{f}\left(\mathbf{z}^{*}, \mathbf{z}^{\tau *}\right)=\partial \mathbf{f}\left(\mathbf{z}, \mathbf{z}^{\tau}\right) /\left.\partial \mathbf{z}\right|_{\left(\mathbf{z}^{*}, \mathbf{z}^{\tau *}\right),} \\
& D_{\mathbf{z}^{\tau}} \mathbf{f}\left(\mathbf{z}^{*}, \mathbf{z}^{\tau *}\right)=\partial \mathbf{f}\left(\mathbf{z}, \mathbf{z}^{\tau}\right) /\left.\partial \mathbf{z}^{\tau}\right|_{\left(\mathbf{z}^{*}, \mathbf{z}^{\tau *}\right)} .
\end{aligned}
$$

Therefore, as discussed before, the eigenvalues of $D \mathbf{f}\left(\mathbf{z}^{*}\right)$ are classified by

$$
\left(n_{1}, n_{2}, n_{3} \mid n_{4}, n_{5}, n_{6}\right)
$$

From the stability and bifurcation theory of dynamical systems at equilibrium, the stability and bifurcation of the periodic solutions can be classified as stated in the theorem. This theorem is proved.

If the Hopf bifurcation of equilibriums of Eq. (110) occurs, there is a periodic solution of the coefficients in Eq. (108) with a frequency $\omega$. Thus, the periodic solution of coefficients can be expressed as

$$
\begin{aligned}
\mathbf{a}_{0}(t)= & \mathbf{A}_{00}(t)+\sum_{m=1}^{M} \mathbf{A}_{0 m}^{(1)}(t) \cos (m \omega t)+\mathbf{A}_{0 m}^{(2)}(t) \sin (m \omega t) \\
\mathbf{b}_{k}(t)= & \mathbf{B}_{k 0}(t)+\sum_{m=1}^{M} \mathbf{B}_{k m}^{(1)}(t) \cos (m \omega t)+\mathbf{B}_{k m}^{(2)}(t) \sin (m \omega t), \\
\mathbf{c}_{k}(t)= & \mathbf{C}_{k 0}(t)+\sum_{m=1}^{M} \mathbf{C}_{k m}^{(1)}(t) \cos (m \omega t)+\mathbf{C}_{k m}^{(2)}(t) \sin (m \omega t), \\
\mathbf{a}_{0}^{\tau}(t)= & \mathbf{A}_{00}^{\tau}(t)+\sum_{m=1}^{M} \mathbf{A}_{0 m}^{\tau(1)}(t) \cos [m \omega(t-\tau)] \\
& +\mathbf{A}_{0 m}^{\tau(2)}(t) \sin [m \omega(t-\tau)] . \\
\mathbf{b}_{k}^{\tau}(t)= & \mathbf{B}_{k 0}^{\tau}(t)+\sum_{m=1}^{M} \mathbf{B}_{k m}^{\tau(1)}(t) \cos [m \omega(t-\tau)] \\
& +\mathbf{B}_{k m}^{\tau(2)}(t) \sin [m \omega(t-\tau)] \\
& +\mathbf{C}_{k 0}^{\tau(t)}(t)+\sum_{m=1}^{M} \mathbf{C}_{k m}^{\tau(1)}(t) \sin [m \omega(t-\tau)] \\
\mathbf{c}_{k}^{\tau}(t) &
\end{aligned}
$$

Substitution of Eq. (119) into Eq. (108) gives

$$
\begin{aligned}
\mathbf{x}^{*}(t)= & \mathbf{A}_{00}(t)+\sum_{m=1}^{M} \mathbf{A}_{0 m}^{(1)}(t) \cos (m \omega t)+\mathbf{A}_{0 m}^{(2)}(t) \sin (m \omega t) \\
& +\sum_{k=1}^{N} \mathbf{B}_{k 0}(t) \cos (k \Omega t)+\mathbf{C}_{k 0}(t) \sin (k \Omega t) \\
& +\sum_{k=1}^{N} \sum_{m=1}^{M} \mathbf{B}_{k m}^{(1)}(t) \cos (m \omega t) \cos (k \Omega t) \\
& +\mathbf{B}_{k m}^{(2)}(t) \sin (m \omega t) \cos (k \Omega t) \\
& +\sum_{k=1}^{N} \sum_{m=1}^{M} \mathbf{C}_{k m}^{(1)}(t) \cos (m \omega t) \sin (k \Omega t) \\
& +\mathbf{C}_{k m}^{(2)}(t) \sin (m \omega t) \sin (k \Omega t)
\end{aligned}
$$

$$
\begin{aligned}
\mathbf{x}^{\tau *}(t)= & \mathbf{A}_{00}^{\tau}(t)+\sum_{m=1}^{M} \mathbf{A}_{0 m}^{\tau(1)}(t) \cos [m \omega(t-\tau)] \\
& +\mathbf{A}_{0 m}^{\tau(2)}(t) \sin [m \omega(t-\tau)] \\
& +\sum_{k=1}^{N} \mathbf{B}_{k 0}^{\tau}(t) \cos [k \Omega(t-\tau)] \\
& +\mathbf{C}_{k 0}^{\tau}(t) \sin [k \Omega(t-\tau)] \\
& +\sum_{k=1}^{N} \sum_{m=1}^{M}\left\{\mathbf{B}_{k m}^{\tau(1)}(t) \cos [m \omega(t-\tau)] \cos [k \Omega(t-\tau)]\right. \\
& \left.+\mathbf{B}_{k m}^{\tau(2)}(t) \sin [m \omega(t-\tau)] \cos [k \Omega(t-\tau)]\right\} \\
& +\sum_{k=1}^{N} \sum_{m=1}^{M}\left\{\mathbf{C}_{k m}^{\tau(1)}(t) \cos [m \omega(t-\tau)] \sin [k \Omega(t-\tau)]\right. \\
& \left.+\mathbf{C}_{k m}^{\tau(2)}(t) \sin [m \omega(t-\tau)] \sin [k \Omega(t-\tau)]\right\}
\end{aligned}
$$

If the new solution is still periodic with excitation period $T=2 \pi / \Omega$, then for specific $m$, the following relation should be satisfied.

$m \omega=k \Omega$.

For this case, $k=1$ should be inserted because $k>1$ terms are already included in the Fourier series expression. Thus, as in dynamical systems without delay,

$m \omega=\Omega$.

For $m=1$, the period-1 flow is obtained and equation (120) will become Eq. (108). For the period- $m$ flow, we have

$$
\begin{aligned}
\mathbf{x}^{(m) *}(t)= & \mathbf{a}_{0}^{(m)}(t)+\sum_{k=1}^{N} \mathbf{b}_{k / m}(t) \cos \left(\frac{k}{m} \Omega t\right) \\
& +\mathbf{c}_{k / m}(t) \sin \left(\frac{k}{m} \Omega t\right) ; \\
\mathbf{x}^{(m) \tau *}(t)= & \mathbf{a}_{0}^{\tau(m)}(t)+\sum_{k=1}^{N} \mathbf{b}_{k / m}^{\tau}(t) \cos \left[\frac{k}{m} \Omega(t-\tau)\right] \\
& +\mathbf{c}_{k / m}^{\tau}(t) \sin \left[\frac{k}{m} \Omega(t-\tau)\right] .
\end{aligned}
$$

If $m \omega \neq k \Omega$ for any $m$ and $k$, the solutions will be quasiperiodic or chaotic instead of periodic in the delayed nonlinear dynamical systems. Herein, we will not discuss about it herein. If period- 1 motion possesses at least $N_{1}$ harmonic vector terms, then the total harmonic vector terms for period$m$ motion should be $N \geq m N_{1}$. The period- $m$ flow in timedelayed, dynamical systems will be discussed as follows.

Theorem 10 Consider a nonlinear dynamical system in Eq. (107). If such dynamical system has a period-m flow $\mathbf{x}^{(m) *}(t)$ with finite norm $\left\|\mathbf{x}^{(m) *}\right\|$ and period $T=2 \pi / \Omega$, there is an analytical expression for the periodic flow of Eq. (107) in the form of 


$$
\begin{aligned}
\mathbf{x}^{(m) *}(t)= & \mathbf{a}_{0}^{(m)}(t)+\sum_{k=1}^{N} \mathbf{b}_{k / m}(t) \cos \left(\frac{k}{m} \Omega t\right) \\
& +\mathbf{c}_{k / m}(t) \sin \left(\frac{k}{m} \Omega t\right) ; \\
\mathbf{x}^{\tau(m) *}(t)= & \mathbf{a}_{0}^{\tau(m)}(t)+\sum_{k=1}^{N} \mathbf{b}_{k / m}^{\tau}(t) \cos \left[\frac{k}{m} \Omega(t-\tau)\right] \\
& +\mathbf{c}_{k / m}^{\tau}(t) \sin \left[\frac{k}{m} \Omega(t-\tau)\right]
\end{aligned}
$$

with for $k=1,2, \ldots, N$

$\mathbf{a}_{0}^{(m)}=\left(a_{01}^{(m)}, a_{02}^{(m)}, \ldots, a_{0 n}^{(m)}\right)^{\mathrm{T}}$,

$\mathbf{b}_{k / m}=\left(b_{k / m 1}, b_{k / m 2}, \ldots, b_{k / m n}\right)^{\mathrm{T}}$,

$\mathbf{c}_{k / m}=\left(c_{k / m 1}, c_{k / m 2}, \ldots, c_{k / m n}\right)^{\mathrm{T}}$;

$\mathbf{a}_{0}^{\tau(m)}=\left(a_{01}^{\tau(m)}, a_{02}^{\tau(m)}, \ldots, a_{0 n}^{\tau(m)}\right)^{\mathrm{T}}$,

$\mathbf{b}_{k / m}^{\tau}=\left(b_{k / m 1}^{\tau}, b_{k / m 2}^{\tau}, \ldots, b_{k / m n}^{\tau}\right)^{\mathrm{T}}$,

$\mathbf{c}_{k / m}^{\tau}=\left(c_{k / m 1}^{\tau}, c_{k / m 2}^{\tau}, \ldots, c_{k / m n}^{\tau}\right)^{\mathrm{T}}$

and the corresponding coefficients slowly varying with time are determined by the following dynamical systems

$\dot{\mathbf{a}}_{0}^{(m)}=\mathbf{F}_{0}^{(m)}\left(\mathbf{z}^{(m)}, \mathbf{z}^{\tau(m)}\right)$,

$\dot{\mathbf{b}}^{(m)}=-\frac{\Omega}{m} \mathbf{k}_{1} \mathbf{c}^{(m)}+\mathbf{F}_{1}^{(m)}\left(\mathbf{z}^{(m)}, \mathbf{z}^{\tau(m)}\right)$,

$\dot{\mathbf{c}}^{(m)}=\frac{\Omega}{m} \mathbf{k}_{1} \mathbf{b}^{(m)}+\mathbf{F}_{2}^{(m)}\left(\mathbf{z}^{(m)}, \mathbf{z}^{\tau(m)}\right)$

where

$\mathbf{k}_{1}=\operatorname{diag}\left(\mathbf{I}_{n \times n}, 2 \mathbf{I}_{n \times n}, \ldots, N \mathbf{I}_{n \times n}\right)$,

$\mathbf{b}^{(m)}=\left(\mathbf{b}_{1 / m}, \mathbf{b}_{2 / m}, \ldots, \mathbf{b}_{N / m}\right)^{\mathrm{T}}$,

$\mathbf{c}^{(m)}=\left(\mathbf{c}_{1 / m}, \mathbf{c}_{2 / m}, \ldots, \mathbf{c}_{N / m}\right)^{\mathrm{T}}$

$\mathbf{b}^{\tau(m)}=\left(\mathbf{b}_{1 / m}^{\tau}, \mathbf{b}_{2 / m}^{\tau}, \ldots, \mathbf{b}_{N / m}^{\tau}\right)^{\mathrm{T}}$,

$\mathbf{c}^{\tau(m)}=\left(\mathbf{c}_{1 / m}^{\tau}, \mathbf{c}_{2 / m}^{\tau}, \ldots, \mathbf{c}_{N / m}^{\tau}\right)^{\mathrm{T}}$;

$\mathbf{F}_{1}^{(m)}=\left(\mathbf{F}_{11}^{(m)}, \mathbf{F}_{12}^{(m)}, \ldots, \mathbf{F}_{1 N}^{(m)}\right)^{\mathrm{T}}$,

$\mathbf{F}_{2}^{(m)}=\left(\mathbf{F}_{21}^{(m)}, \mathbf{F}_{22}^{(m)}, \ldots, \mathbf{F}_{2 N}^{(m)}\right)^{\mathrm{T}}$

$\mathbf{z}^{(m)}=\left(\mathbf{a}_{0}^{(m)}, \mathbf{b}^{(m)}, \mathbf{c}^{(m)}\right)^{\mathrm{T}}$,

$\mathbf{z}^{\tau(m)}=\left(\mathbf{a}_{0}^{\tau(m)}, \mathbf{b}^{\tau(m)}, \mathbf{c}^{\tau(m)}\right)^{\mathrm{T}}$

for $N=1,2, \ldots, \infty$;

and

$$
\begin{aligned}
& \mathbf{F}_{0}^{(m)}\left(\mathbf{z}^{(m)}, \mathbf{z}^{\tau(m)}\right)=\frac{1}{m T} \int_{0}^{m T} \mathbf{f}\left(\mathbf{x}^{(m) *}, \mathbf{x}^{\tau(m) *}, \mathbf{p}\right) d t ; \\
& \mathbf{F}_{1 k}^{(m)}\left(\mathbf{z}^{(m)}, \mathbf{z}^{\tau(m)}\right) \\
& =\frac{2}{m T} \int_{0}^{m T} \mathbf{f}\left(\mathbf{x}^{(m) *}, \mathbf{x}^{\tau(m) *}, \mathbf{p}\right) \cos \left(\frac{k}{m} \Omega t\right) d t,
\end{aligned}
$$

$$
\begin{aligned}
& \mathbf{F}_{2 k}^{(m)}\left(\mathbf{z}^{(m)}, \mathbf{z}^{\tau(m)}\right) \\
& \quad=\frac{2}{m T} \int_{0}^{m T} \mathbf{f}\left(\mathbf{x}^{(m) *}, \mathbf{x}^{\tau(m) *}, \mathbf{p}\right) \sin \left(\frac{k}{m} \Omega t\right) d t \\
& \quad \text { for } k=1,2, \ldots, N .
\end{aligned}
$$

Equation (126) becomes

$\dot{\mathbf{z}}^{(m)}=\mathbf{f}^{(m)}\left(\mathbf{z}^{(m)}, \mathbf{z}^{\tau(m)}\right)$

where

$\mathbf{f}^{(m)}=\left(\mathbf{F}_{0}^{(m)},-\frac{\Omega}{m} \mathbf{k}_{1} \mathbf{c}^{(m)}+\mathbf{F}_{1}^{(m)}, \frac{\Omega}{m} \mathbf{k}_{1} \mathbf{b}^{(m)}+\mathbf{F}_{2}^{(m)}\right)^{\mathrm{T}}$

If equilibrium $\left(\mathbf{z}^{(m) *}=\mathbf{z}^{\tau(m) *}\right)$ of Eq. (129) (i.e., $\mathbf{f}^{(m)}\left(\mathbf{z}^{(m) *}\right.$, $\left.\left.\mathbf{z}^{\tau(m) *}\right)=\mathbf{0}\right)$ exists, then the analytical solution of period- $m$ flows of Eq. (107) exists in Eq. (124). In the vicinity of equilib$\operatorname{rium} \mathbf{z}^{(m) *}=\mathbf{z}^{\tau(m) *}$, with $\mathbf{z}^{(m)}=\mathbf{z}^{(m) *}+\Delta \mathbf{z}^{(m)}$ and $\mathbf{z}^{\tau(m)}=$ $\mathbf{z}^{\tau(m) *}+\Delta \mathbf{z}^{\tau(m)}$, the linearized equation of $E q$. (129) is

$$
\begin{aligned}
\Delta \dot{\mathbf{z}}^{(m)}= & D_{\mathbf{z}^{(m)}} \mathbf{f}^{(m)}\left(\mathbf{z}^{(m) *}, \mathbf{z}^{\tau(m) *}\right) \Delta \mathbf{z}^{(m)} \\
& +D_{\mathbf{z}^{\tau(m)}} \mathbf{f}^{(m)}\left(\mathbf{z}^{(m) *}, \mathbf{z}^{\tau(m) *}\right) \Delta \mathbf{z}^{\tau(m)}
\end{aligned}
$$

and the eigenvalue analysis of equilibrium $\mathbf{z}^{(m) *}=\mathbf{z}^{\tau(m) *}$ is given by

$$
\begin{aligned}
& \mid D_{\mathbf{z}^{(m)}} \mathbf{f}^{(m)}\left(\mathbf{z}^{(m) *}, \mathbf{z}^{\tau(m) *}\right)-\lambda \mathbf{I}_{n(2 N+1) \times n(2 N+1)} \\
& \quad+D_{\mathbf{z}^{\tau(m)}} \mathbf{f}^{(m)}\left(\mathbf{z}^{(m) *}, \mathbf{z}^{\tau(m) *}\right) e^{-\lambda \tau} \mid=0
\end{aligned}
$$

where

$$
\begin{aligned}
& D_{\mathbf{z}^{(m)}} \mathbf{f}^{(m)}\left(\mathbf{z}^{(m) *}, \mathbf{z}^{\tau(m) *}\right) \\
& \quad=\partial \mathbf{f}^{(m)}\left(\mathbf{z}^{(m)}, \mathbf{z}^{\tau(m)}\right) /\left.\partial \mathbf{z}^{(m)}\right|_{\left(\mathbf{z}^{(m) *}, \mathbf{z}^{\tau(m) *)}\right.}, \\
& D_{\mathbf{z}^{\tau(m)}} \mathbf{f}^{(m)}\left(\mathbf{z}^{(m) *}, \mathbf{z}^{\tau(m) *}\right) \\
& \quad=\partial \mathbf{f}^{(m)}\left(\mathbf{z}^{(m)}, \mathbf{z}^{\tau(m)}\right) /\left.\partial \mathbf{z}^{\tau(m)}\right|_{\left(\mathbf{z}^{(m) *}, \mathbf{z}^{\tau(m) *}\right)} .
\end{aligned}
$$

The stability and bifurcation of periodic solution can be classified by the eigenvalues of Eq. (131) at equilibrium $\mathbf{z}^{(m) *}=\mathbf{z}^{\tau(m) *}$ with

$\left(n_{1}, n_{2}, n_{3} \mid n_{4}, n_{5}, n_{6}\right)$.

(i) If all eigenvalues of the equilibrium possess negative real parts, the approximate periodic solution is stable.

(ii) If at least one of eigenvalues of the equilibrium possesses positive real part, the approximate periodic solution is unstable.

(iii) The boundaries between stable and unstable equilibriums with higher order singularity give bifurcation and stability conditions with higher order singularity.

Proof The proof is similar to the proof of Theorem 9. Since $\mathbf{f}\left(\mathbf{x}, \mathbf{x}^{\tau}, \mathbf{p}\right)$ is a $C^{r}$-continuous nonlinear function vector ( $r \geq 1)$, the velocity $\dot{\mathbf{x}}$ should be $C^{r}$-continuous $(r \geq 1)$, and then the acceleration $\ddot{\mathbf{x}}$ should be bounded (i.e., $\|\ddot{\mathbf{x}}\| \leq K$ ). 
Thus, the Fourier series expression of a period- $m$ flow in Eq. (124) is convergent from the Fourier series theory. Taking the derivative of Eq. (124) with respect to time gives

$$
\begin{aligned}
\dot{\mathbf{x}}^{(m) *}(t)= & \dot{\mathbf{a}}_{0}^{(m)}+\sum_{k=1}^{N}\left[\dot{\mathbf{b}}_{k / m}+\frac{k}{m} \Omega \mathbf{c}_{k / m}\right] \cos \left(\frac{k}{m} \Omega t\right) \\
& +\left[\dot{\mathbf{c}}_{k / m}-\frac{k}{m} \Omega \mathbf{b}_{k / m}\right] \sin \left(\frac{k}{m} \Omega t\right), \\
\dot{\mathbf{x}}^{(m) \tau *}(t)= & \dot{\mathbf{a}}_{0}^{\tau(m)}+\sum_{k=1}^{N}\left\{\left[\dot{\mathbf{b}}_{k / m}^{\tau}+\frac{k}{m} \Omega \mathbf{c}_{k / m}^{\tau}\right] \cos \left[\frac{k}{m} \Omega(t-\tau)\right]\right. \\
& \left.+\left[\dot{\mathbf{c}}_{k / m}^{\tau}-\frac{k}{m} \Omega \mathbf{b}_{k / m}^{\tau}\right] \sin \left[\frac{k}{m} \Omega(t-\tau)\right]\right\} .
\end{aligned}
$$

Since the coefficients vary slowly with time, substitution of the foregoing equation into Eq. (107) and averaging for constant, $\cos (k \Omega t / m)$ and $\sin (k \Omega t / m)(k=1,2, \ldots)$ yields for $k=1,2, \ldots, N$

$$
\begin{aligned}
& \dot{\mathbf{a}}_{0}^{(m)}=\mathbf{F}_{0}\left(\mathbf{z}^{(m)}, \mathbf{z}^{\tau(m)}\right), \\
& \dot{\mathbf{b}}_{k / m}+\frac{\Omega}{m} k \mathbf{c}_{k / m}=\mathbf{F}_{1 k}^{(m)}\left(\mathbf{z}^{(m)}, \mathbf{z}^{\tau(m)}\right), \\
& \dot{\mathbf{c}}_{k / m}-\frac{\Omega}{m} k \mathbf{b}_{k / m}=\mathbf{F}_{2 k}^{(m)}\left(\mathbf{z}^{(m)}, \mathbf{z}^{\tau(m)}\right) .
\end{aligned}
$$

where

$$
\begin{aligned}
& \mathbf{F}_{0}^{(m)}\left(\mathbf{z}^{(m)}, \mathbf{z}^{\tau(m)}\right) \\
& =\frac{1}{m T} \int_{0}^{m T} \mathbf{f}\left(\mathbf{x}^{(m) *}, \mathbf{x}^{\tau(m) *}, \mathbf{p}\right) d t ; \\
& \mathbf{F}_{1 k}^{(m)}\left(\mathbf{z}^{(m)}, \mathbf{z}^{\tau(m)}\right) \\
& =\frac{2}{m T} \int_{0}^{m T} \mathbf{f}\left(\mathbf{x}^{(m) *}, \mathbf{x}^{\tau(m) *}, \mathbf{p}\right) \cos \left(\frac{k}{m} \Omega t\right) d t, \\
& \mathbf{F}_{2 k}^{(m)}\left(\mathbf{z}^{(m)}, \mathbf{z}^{\tau(m)}\right) \\
& \quad=\frac{2}{m T} \int_{0}^{m T} \mathbf{f}\left(\mathbf{x}^{(m) *}, \mathbf{x}^{\tau(m) *}, \mathbf{p}\right) \sin \left(\frac{k}{m} \Omega t\right) d t
\end{aligned}
$$

for $k=1,2, \ldots, N$

and

$$
\begin{aligned}
& \mathbf{k}_{1}=\operatorname{diag}\left(\mathbf{I}_{n \times n}, 2 \mathbf{I}_{n \times n}, \ldots, N \mathbf{I}_{n \times n}\right), \\
& \mathbf{b}^{(m)}=\left(\mathbf{b}_{1 / m}, \mathbf{b}_{2 / m}, \ldots, \mathbf{b}_{N / m}\right)^{\mathrm{T}}, \\
& \mathbf{c}^{(m)}=\left(\mathbf{c}_{1 / m}, \mathbf{c}_{2 / m}, \ldots, \mathbf{c}_{N / m}\right)^{\mathrm{T}} ; \\
& \mathbf{b}^{\tau(m)}=\left(\mathbf{b}_{1 / m}^{\tau}, \mathbf{b}_{2 / m}^{\tau}, \ldots, \mathbf{b}_{N / m}^{\tau}\right)^{\mathrm{T}}, \\
& \mathbf{c}^{\tau(m)}=\left(\mathbf{c}_{1 / m}^{\tau}, \mathbf{c}_{2 / m}^{\tau}, \ldots, \mathbf{c}_{N / m}^{\tau}\right)^{\mathrm{T}} ; \\
& \mathbf{F}_{1}^{(m)}=\left(\mathbf{F}_{11}^{(m)}, \mathbf{F}_{12}^{(m)}, \ldots, \mathbf{F}_{1 N}^{(m)}\right)^{\mathrm{T}}, \\
& \mathbf{F}_{2}^{(m)}=\left(\mathbf{F}_{21}^{(m)}, \mathbf{F}_{22}^{(m)}, \ldots, \mathbf{F}_{2 N}^{(m)}\right)^{\mathrm{T}} ; \\
& \mathbf{z}^{(m)}=\left(\mathbf{a}_{0}^{(m)}, \mathbf{b}^{(m)}, \mathbf{c}^{(m)}\right)^{\mathrm{T}},
\end{aligned}
$$

$\mathbf{z}^{\tau(m)}=\left(\mathbf{a}_{0}^{\tau(m)}, \mathbf{b}^{\tau(m)}, \mathbf{c}^{\tau(m)}\right)^{\mathrm{T}}$

for $N=1,2, \ldots, \infty$.

Rearranging the foregoing equation gives Eq. (126), i.e.,

$\dot{\mathbf{a}}_{0}^{(m)}=\mathbf{F}_{0}^{(m)}\left(\mathbf{z}^{(m)}, \mathbf{z}^{\tau(m)}\right)$,

$\dot{\mathbf{b}}^{(m)}=-\frac{\Omega}{m} \mathbf{k}_{1} \mathbf{c}^{(m)}+\mathbf{F}_{1}^{(m)}\left(\mathbf{z}^{(m)}, \mathbf{z}^{\tau(m)}\right)$,

$\dot{\mathbf{c}}^{(m)}=\frac{\Omega}{m} \mathbf{k}_{1} \mathbf{b}^{(m)}+\mathbf{F}_{2}^{(m)}\left(\mathbf{z}^{(m)}, \mathbf{z}^{\tau(m)}\right)$.

Introducing

$\mathbf{f}^{(m)}=\left(\mathbf{F}_{0}^{(m)},-\Omega \mathbf{k}_{1} \mathbf{c}^{(m)} / m+\mathbf{F}_{1}^{(m)}, \Omega \mathbf{k}_{1} \mathbf{b}^{(m)} / m+\mathbf{F}_{2}^{(m)}\right)^{\mathrm{T}}$, the standard form of equation in Eq. (126) becomes

$\dot{\mathbf{z}}^{(m)}=\mathbf{f}^{(m)}\left(\mathbf{z}^{(m)}, \mathbf{z}^{\tau(m)}\right)$

Equilibriums $\mathbf{z}^{(m) *}=\mathbf{z}^{\tau(m) *}$ of the foregoing equation are given by $\mathbf{f}^{(m)}\left(\mathbf{z}^{(m) *}, \mathbf{z}^{\tau(m) *}\right)=\mathbf{0}$, i.e.,

$$
\begin{aligned}
\mathbf{0}= & \mathbf{F}_{0}^{(m)}\left(\mathbf{a}_{0}^{(m) *}, \mathbf{b}^{(m) *}, \mathbf{c}^{(m) *} ; \mathbf{a}_{0}^{\tau(m) *}, \mathbf{b}^{\tau(m) *}, \mathbf{c}^{\tau(m) *}\right), \\
\mathbf{0}= & -\frac{\Omega}{m} \mathbf{k}_{1} \mathbf{c}^{(m) *} \\
& +\mathbf{F}_{1}^{(m)}\left(\mathbf{a}_{0}^{(m) *}, \mathbf{b}^{(m) *}, \mathbf{c}^{(m) *} ; \mathbf{a}_{0}^{\tau(m) *}, \mathbf{b}^{\tau(m) *}, \mathbf{c}^{\tau(m) *}\right), \\
\mathbf{0}= & \frac{\Omega}{m} \mathbf{k}_{1} \mathbf{b}^{(m) *} \\
& +\mathbf{F}_{2}^{(m)}\left(\mathbf{a}_{0}^{(m) *}, \mathbf{b}^{(m) *}, \mathbf{c}^{(m) *} ; \mathbf{a}_{0}^{\tau(m) *}, \mathbf{b}^{\tau(m) *}, \mathbf{c}^{\tau(m) *}\right)
\end{aligned}
$$

with

$\mathbf{a}_{0}^{(m) *}=\mathbf{a}_{0}^{\tau(m) *}, \mathbf{b}^{(m) *}=\mathbf{b}^{\tau(m) *}, \mathbf{c}^{(m) *}=\mathbf{c}^{\tau(m) *}$.

Thus, the existing conditions of solutions of the forgoing equation are the conditions of the periodic solutions for nonlinear dynamical systems. The foregoing equation gives equilibrium $\mathbf{z}^{(m) *}=\mathbf{z}^{\tau(m) *}$. In vicinity of $\left(\mathbf{z}^{(m) *}, \mathbf{z}^{\tau(m) *}\right)$, with $\mathbf{z}^{(m)}=\mathbf{z}^{(m) *}+\Delta \mathbf{z}^{(m)}$ and $\mathbf{z}^{\tau(m)}=\mathbf{z}^{\tau(m) *}+\Delta \mathbf{z}^{\tau(m)}$, the linearized equation of $\dot{\mathbf{z}}^{(m)}=\mathbf{f}^{(m)}\left(\mathbf{z}^{(m)}, \mathbf{z}^{\tau(m)}\right)$ is

$$
\begin{aligned}
\Delta \dot{\mathbf{z}}^{(m)}= & D_{\mathbf{z}^{(m) *}} \mathbf{f}^{(m)}\left(\mathbf{z}^{(m)}, \mathbf{z}^{\tau(m) *}\right) \Delta \mathbf{z}^{(m)} \\
& +D_{\mathbf{z}^{\tau(m)}} \mathbf{f}^{(m)}\left(\mathbf{z}^{(m) *}, \mathbf{z}^{\tau(m) *}\right) \Delta \mathbf{z}^{\tau(m)}
\end{aligned}
$$

and the eigenvalue analysis of equilibrium $\mathbf{z}^{(m) *}=\mathbf{z}^{\tau(m) *}$ is completed via

$$
\begin{gathered}
\mid D_{\mathbf{z}^{(m)}} \mathbf{f}^{(m)}\left(\mathbf{z}^{(m) *}, \mathbf{z}^{\tau(m) *}\right)-\lambda \mathbf{I}_{n(2 N+1) \times n(2 N+1)} \\
\quad+D_{\mathbf{z}^{\tau(m)}} \mathbf{f}^{(m)}\left(\mathbf{z}^{(m) *}, \mathbf{z}^{\tau(m) *}\right) e^{-\lambda \tau} \mid=0
\end{gathered}
$$

where

$$
\begin{aligned}
& D_{\mathbf{z}^{(m)}} \mathbf{f}^{(m)}\left(\mathbf{z}^{(m) *}, \mathbf{z}^{\tau(m) *}\right) \\
& \quad=\partial \mathbf{f}^{(m)}\left(\mathbf{z}^{(m)}, \mathbf{z}^{\tau(m)}\right) /\left.\partial \mathbf{z}^{(m)}\right|_{\left(\mathbf{z}^{(m) *}, \mathbf{z}^{\tau(m) *}\right)},
\end{aligned}
$$




$$
\begin{aligned}
& D_{\mathbf{z}^{\tau(m)}} \mathbf{f}^{(m)}\left(\mathbf{z}^{(m) *}, \mathbf{z}^{\tau(m) *}\right) \\
& \quad=\partial \mathbf{f}^{(m)}\left(\mathbf{z}^{(m)}, \mathbf{z}^{\tau(m)}\right) /\left.\partial \mathbf{z}^{\tau(m)}\right|_{\left(\mathbf{z}^{(m) *}, \mathbf{z}^{\tau(m) *}\right)} \cdot
\end{aligned}
$$

Thus, the corresponding eigenvalues are classified by

$\left(n_{1}, n_{2}, n_{3} \mid n_{4}, n_{5}, n_{6}\right)$

From the stability and bifurcation theory of dynamical systems at equilibrium, the stability and bifurcation of the approximate periodic solutions can be classified as stated in the theorem. This theorem is proved.

If $m \rightarrow \infty$ with $N \rightarrow \infty$, Eq. (124) will give the analytical expression of chaos in time-delayed dynamical systems in Eq. (107). The route from the periodic flow to chaos is through the Hopf bifurcation.

\subsection{Periodically forced, time-delayed nonlinear systems}

Periodic flows in periodically forced, time-delayed, nonlinear dynamical systems will be discussed herein. If a timedelayed system has periodic flows with an external period $T=2 \pi / \Omega$, such periodic flows can be expressed through the Fourier series, discussed as follows.

Theorem 11 Consider a periodically forced, time-delayed, nonlinear dynamical system as

$\dot{\mathbf{x}}=\mathbf{F}\left(\mathbf{x}, \mathbf{x}^{\tau}, t, \mathbf{p}\right) \in \mathbf{R}^{n}$

where $\mathbf{F}\left(\mathbf{x}, \mathbf{x}^{\tau}, t, \mathbf{p}\right)$ is a $C^{r}$-continuous nonlinear function vector ( $r \geq 1)$ with forcing period $T=2 \pi / \Omega$. If there is a periodic flow $\mathbf{x}^{*}(t)$ with finite norm $\left\|\mathbf{x}^{*}\right\|$, an analytical expression for the periodic flow of Eq. (135) is in the form of

$$
\begin{aligned}
\mathbf{x}^{*}= & \mathbf{a}_{0}(t)+\sum_{k=1}^{N} \mathbf{b}_{k}(t) \cos (k \Omega t)+\mathbf{c}_{k}(t) \sin (k \Omega t), \\
\mathbf{x}^{\tau *}= & \mathbf{a}_{0}^{\tau}(t)+\sum_{k=1}^{N} \mathbf{b}_{k}^{\tau}(t) \cos [k \Omega(t-\tau)] \\
& +\mathbf{c}_{k}^{\tau}(t) \sin [k \Omega(t-\tau)]
\end{aligned}
$$

with

$$
\begin{aligned}
\mathbf{a}_{0} & =\left(a_{01}, a_{02}, \ldots, a_{0 n}\right)^{\mathrm{T}}, \\
\mathbf{b}_{k} & =\left(b_{k 1}, b_{k 2}, \ldots, b_{k n}\right)^{\mathrm{T}}, \\
\mathbf{c}_{k} & =\left(c_{k 1}, c_{k 2}, \ldots, c_{k n}\right)^{\mathrm{T}} ; \\
\mathbf{a}_{0}^{\tau} & =\left(a_{01}^{\tau}, a_{02}^{\tau}, \ldots, a_{0 n}^{\tau}\right)^{\mathrm{T}}, \\
\mathbf{b}_{k}^{\tau} & =\left(b_{k 1}^{\tau}, b_{k 2}^{\tau}, \ldots, b_{k n}^{\tau}\right)^{\mathrm{T}}, \\
\mathbf{c}_{k}^{\tau} & =\left(c_{k 1}^{\tau}, c_{k 2}^{\tau}, \ldots, c_{k n}^{\tau}\right)^{\mathrm{T}} ;
\end{aligned}
$$

and the corresponding coefficients slowly varying with time are determined by

$$
\begin{aligned}
& \dot{\mathbf{a}}_{0}=\mathbf{F}_{0}\left(\mathbf{z}, \mathbf{z}^{\tau}\right), \\
& \dot{\mathbf{b}}=-\Omega \mathbf{k}_{1} \mathbf{c}+\mathbf{F}_{1}\left(\mathbf{z}, \mathbf{z}^{\tau}\right), \\
& \dot{\mathbf{c}}=\Omega \mathbf{k}_{1} \mathbf{b}+\mathbf{F}_{2}\left(\mathbf{z}, \mathbf{z}^{\tau}\right) ;
\end{aligned}
$$

where

$$
\begin{aligned}
& \mathbf{k}_{1}=\operatorname{diag}\left(\mathbf{I}_{n \times n}, 2 \mathbf{I}_{n \times n}, \ldots, N \mathbf{I}_{n \times n}\right), \\
& \mathbf{b}=\left(\mathbf{b}_{1}, \mathbf{b}_{2}, \ldots, \mathbf{b}_{N}\right)^{\mathrm{T}} \\
& \mathbf{c}=\left(\mathbf{c}_{1}, \mathbf{c}_{2}, \ldots, \mathbf{c}_{N}\right)^{\mathrm{T}} \\
& \mathbf{b}^{\tau}=\left(\mathbf{b}_{1}^{\tau}, \mathbf{b}_{2}^{\tau}, \ldots, \mathbf{b}_{N}^{\tau}\right)^{\mathrm{T}} \\
& \mathbf{c}^{\tau}=\left(\mathbf{c}_{1}^{\tau}, \mathbf{c}_{2}^{\tau}, \ldots, \mathbf{c}_{N}^{\tau}\right)^{\mathrm{T}} \\
& \mathbf{F}_{1}=\left(\mathbf{F}_{11}, \mathbf{F}_{12}, \ldots, \mathbf{F}_{1 N}\right)^{\mathrm{T}} \\
& \mathbf{F}_{2}=\left(\mathbf{F}_{21}, \mathbf{F}_{22}, \ldots, \mathbf{F}_{2 N}\right)^{\mathrm{T}} ; \\
& \mathbf{z}=\left(\mathbf{a}_{0}, \mathbf{b}, \mathbf{c}\right)^{\mathrm{T}}, \\
& \mathbf{z}^{\tau}=\left(\mathbf{a}_{0}^{\tau}, \mathbf{b}^{\tau}, \mathbf{c}^{\tau}\right)^{\mathrm{T}} \\
& \text { for } \quad N=1,2, \ldots, \infty \\
& \text { and for } k=1,2, \ldots, N
\end{aligned}
$$

$$
\mathbf{F}_{0}\left(\mathbf{z}, \mathbf{z}^{\tau}\right)=\frac{1}{T} \int_{0}^{T} \mathbf{F}\left(\mathbf{x}^{*}, \mathbf{x}^{\tau *}, t, \mathbf{p}\right) d t
$$

$\mathbf{F}_{1 k}\left(\mathbf{z}, \mathbf{z}^{\tau}\right)=\frac{2}{T} \int_{0}^{T} \mathbf{F}\left(\mathbf{x}^{*}, \mathbf{x}^{\tau *}, t, \mathbf{p}\right) \cos (k \Omega t) d t$,

$\mathbf{F}_{2 k}\left(\mathbf{z}, \mathbf{z}^{\tau}\right)=\frac{2}{T} \int_{0}^{T} \mathbf{F}\left(\mathbf{x}^{*}, \mathbf{x}^{\tau *}, t, \mathbf{p}\right) \sin (k \Omega t) d t$.

Equation (138) becomes.

$\dot{\mathbf{z}}=\mathbf{f}\left(\mathbf{z}, \mathbf{z}^{\tau}\right)$

where

$\mathbf{f}=\left(\mathbf{F}_{0},-\Omega \mathbf{k}_{1} \mathbf{c}+\mathbf{F}_{1}, \Omega \mathbf{k}_{1} \mathbf{c}+\mathbf{F}_{2}\right)^{\mathrm{T}}$

If equilibrium $\mathbf{z}^{*}=\mathbf{z}^{\tau *}$ of Eq. (141) (i.e., $\boldsymbol{f}\left(\mathbf{z}^{*}, \mathbf{z}^{\tau *}\right)=\mathbf{0}$ ) exists, then the approximate analytical solution of periodic flow of Eq. (135) exists in Eq. (136). In vicinity of equilibrium $\mathbf{z}^{*}=\mathbf{z}^{\tau *}$, with $\mathbf{z}=\mathbf{z}^{*}+\Delta \mathbf{z}$ and $\mathbf{z}^{\tau}=\mathbf{z}^{* \tau}+\Delta \mathbf{z}^{\tau}$ the linearized equation of $E q$. (141) is

$\Delta \dot{\mathbf{z}}=D_{\mathbf{z}} \mathbf{f}\left(\mathbf{z}^{*}, \mathbf{z}^{\tau *}\right) \Delta \mathbf{z}+D_{\mathbf{z}^{\tau}} \mathbf{f}\left(\mathbf{z}^{*}, \mathbf{z}^{\tau *}\right) \Delta \mathbf{z}^{\tau}$

and the eigenvalue analysis of the equilibrium $\mathbf{z}^{*}$ is given by

$$
\begin{aligned}
& \mid D_{\mathbf{z}} \mathbf{f}\left(\mathbf{z}^{*}, \mathbf{z}^{\tau *}\right)-\lambda \mathbf{I}_{n(2 N+1) \times n(2 N+1)} \\
& \quad+D_{\mathbf{z}^{\tau}} \mathbf{f}\left(\mathbf{z}^{*}, \mathbf{z}^{\tau *}\right) e^{-\lambda \tau} \mid=0
\end{aligned}
$$

where

$$
\begin{aligned}
& D_{\mathbf{z}} \mathbf{f}\left(\mathbf{z}^{*}, \mathbf{z}^{\tau *}\right)=\partial \mathbf{f}\left(\mathbf{z}, \mathbf{z}^{\tau}\right) /\left.\partial \mathbf{z}\right|_{\left(\mathbf{z}^{*}, \mathbf{z}^{\tau *}\right)}, \\
& D_{\mathbf{z}^{\tau}} \mathbf{f}\left(\mathbf{z}^{*}, \mathbf{z}^{\tau *}\right)=\partial \mathbf{f}\left(\mathbf{z}, \mathbf{z}^{\tau}\right) /\left.\partial \mathbf{z}^{\tau}\right|_{\left(\mathbf{z}^{*}, \mathbf{z}^{\tau *}\right)} .
\end{aligned}
$$

Thus, the stability and bifurcation of periodic solutions can be classified by the eigenvalues of Eq. (143) at equilibrium $\mathbf{z}^{*}=\mathbf{z}^{\tau *}$ with

$\left(n_{1}, n_{2}, n_{3} \mid n_{4}, n_{5}, n_{6}\right)$. 
(i) If all eigenvalues of the equilibrium possess negative real parts, the approximate periodic solution is stable.

(ii) If at least one of eigenvalues of the equilibrium possesses positive real part, the approximate periodic solution is unstable.

(iii) The boundaries between stable and unstable equilibriums with higher order singularity give bifurcation and stability conditions with higher order singularity.

Proof The proof of this theorem is similar to Theorem 9.

Similarly, period- $m$ flows in periodically excited, timedelayed dynamical system in Eq. (135) can be discussed.

Theorem 12 Consider a periodically forced, time-delayed, nonlinear dynamical system in Eq. (135) with a forcing period $T=2 \pi / \Omega$. If there is a period- $m$ flow $\mathbf{x}^{(m) *}(t)$ with finite $\left\|\mathbf{x}^{(m) *}\right\|$, an analytical expression for the period $-m$ flow of Eq. (135) is

$$
\begin{aligned}
\mathbf{x}^{(m) *}(t)= & \mathbf{a}_{0}^{(m)}(t)+\sum_{k=1}^{N} \mathbf{b}_{k / m}(t) \cos \left(\frac{k}{m} \Omega t\right) \\
& +\mathbf{c}_{k / m}(t) \sin \left(\frac{k}{m} \Omega t\right) ; \\
\mathbf{x}^{\tau(m) *}(t)= & \mathbf{a}_{0}^{\tau(m)}(t)+\sum_{k=1}^{N} \mathbf{b}_{k / m}^{\tau}(t) \cos \left[\frac{k}{m} \Omega(t-\tau)\right] \\
& \quad+\mathbf{c}_{k / m}^{\tau}(t) \sin \left[\frac{k}{m} \Omega(t-\tau)\right]
\end{aligned}
$$

with for $k=1,2, \ldots, N$

$\mathbf{a}_{0}^{(m)}=\left(a_{01}^{(m)}, a_{02}^{(m)}, \ldots, a_{0 n}^{(m)}\right)^{\mathrm{T}}$,

$\mathbf{b}_{k / m}=\left(b_{k / m 1}, b_{k / m 2}, \ldots, b_{k / m n}\right)^{\mathrm{T}}$,

$\mathbf{c}_{k / m}=\left(c_{k / m 1}, c_{k / m 2}, \ldots, c_{k / m n}\right)^{\mathrm{T}}$;

$\mathbf{a}_{0}^{\tau(m)}=\left(a_{01}^{\tau(m)}, a_{02}^{\tau(m)}, \ldots, a_{0 n}^{\tau(m)}\right)^{\mathrm{T}}$,

$\mathbf{b}_{k / m}^{\tau}=\left(b_{k / m 1}^{\tau}, b_{k / m 2}^{\tau}, \ldots, b_{k / m n}^{\tau}\right)^{\mathrm{T}}$,

$\mathbf{c}_{k / m}^{\tau}=\left(c_{k / m 1}^{\tau}, c_{k / m 2}^{\tau}, \ldots, c_{k / m n}^{\tau}\right)^{\mathrm{T}}$.

and the corresponding coefficients slowly varying with time are determined by the following dynamical systems

$$
\begin{aligned}
\dot{\mathbf{a}}_{0}^{(m)} & =\mathbf{F}_{0}^{(m)}\left(\mathbf{z}^{(m)}, \mathbf{z}^{\tau(m)}\right), \\
\dot{\mathbf{b}}^{(m)} & =-\frac{\Omega}{m} \mathbf{k}_{1} \mathbf{c}^{(m)}+\mathbf{F}_{1}^{(m)}\left(\mathbf{z}^{(m)}, \mathbf{z}^{\tau(m)}\right), \\
\dot{\mathbf{c}}^{(m)} & =\frac{\Omega}{m} \mathbf{k}_{1} \mathbf{b}^{(m)}+\mathbf{F}_{2}^{(m)}\left(\mathbf{z}^{(m)}, \mathbf{z}^{\tau(m)}\right)
\end{aligned}
$$

where for $N=1,2, \ldots, \infty$

$\mathbf{k}_{1}=\operatorname{diag}\left(\mathbf{I}_{n \times n}, 2 \mathbf{I}_{n \times n}, \ldots, N \mathbf{I}_{n \times n}\right)$,

$\mathbf{b}^{(m)}=\left(\mathbf{b}_{1 / m}, \mathbf{b}_{2 / m}, \ldots, \mathbf{b}_{N / m}\right)^{\mathrm{T}}$,

$\mathbf{c}^{(m)}=\left(\mathbf{c}_{1 / m}, \mathbf{c}_{2 / m}, \ldots, \mathbf{c}_{N / m}\right)^{\mathrm{T}}$;

$\mathbf{b}^{\tau(m)}=\left(\mathbf{b}_{1 / m}^{\tau}, \mathbf{b}_{2 / m}^{\tau}, \ldots, \mathbf{b}_{N / m}^{\tau}\right)^{\mathrm{T}}$,

$\mathbf{c}^{\tau(m)}=\left(\mathbf{c}_{1 / m}^{\tau}, \mathbf{c}_{2 / m}^{\tau}, \ldots, \mathbf{c}_{N / m}^{\tau}\right)^{\mathrm{T}}$

$\mathbf{F}_{1}^{(m)}=\left(\mathbf{F}_{11}^{(m)}, \mathbf{F}_{12}^{(m)}, \ldots, \mathbf{F}_{1 N}^{(m)}\right)^{\mathrm{T}}$,

$\mathbf{F}_{2}^{(m)}=\left(\mathbf{F}_{21}^{(m)}, \mathbf{F}_{22}^{(m)}, \ldots, \mathbf{F}_{2 N}^{(m)}\right)^{\mathrm{T}} ;$

$\mathbf{z}^{(m)}=\left(\mathbf{a}_{0}^{(m)}, \mathbf{b}^{(m)}, \mathbf{c}^{(m)}\right)^{\mathrm{T}}$,

$\mathbf{z}^{\tau(m)}=\left(\mathbf{a}_{0}^{\tau(m)}, \mathbf{b}^{\tau(m)}, \mathbf{c}^{\tau(m)}\right)^{\mathrm{T}}$

for $N=1,2, \ldots, \infty$;

and

$$
\begin{aligned}
& \mathbf{F}_{0}^{(m)}\left(\mathbf{z}^{(m)}, \mathbf{z}^{\tau(m)}\right)=\frac{1}{m T} \int_{0}^{m T} \mathbf{f}\left(\mathbf{x}^{(m) *}, \mathbf{x}^{\tau(m) *}, t, \mathbf{p}\right) d t \\
& \mathbf{F}_{1 k}^{(m)}\left(\mathbf{z}^{(m)}, \mathbf{z}^{\tau(m)}\right) \\
& =\frac{2}{m T} \int_{0}^{m T} \mathbf{f}\left(\mathbf{x}^{(m) *}, \mathbf{x}^{\tau(m) *}, t, \mathbf{p}\right) \cos \left(\frac{k}{m} \Omega t\right) d t \\
& \mathbf{F}_{2 k}^{(m)}\left(\mathbf{z}^{(m)}, \mathbf{z}^{\tau(m)}\right) \\
& =\frac{2}{m T} \int_{0}^{m T} \mathbf{f}\left(\mathbf{x}^{(m) *}, \mathbf{x}^{\tau(m) *}, t, \mathbf{p}\right) \sin \left(\frac{k}{m} \Omega t\right) d t
\end{aligned}
$$

for $k=1,2, \ldots, N$.

Equation (149) becomes

$\dot{\mathbf{z}}^{(m)}=\mathbf{f}^{(m)}\left(\mathbf{z}^{(m)}, \mathbf{z}^{\tau(m)}\right)$

where

$\mathbf{f}^{(m)}=\left(\mathbf{F}_{0}^{(m)},-\frac{\Omega}{m} \mathbf{k}_{1} \mathbf{c}^{(m)}+\mathbf{F}_{1}^{(m)}, \frac{\Omega}{m} \mathbf{k}_{1} \mathbf{b}^{(m)}+\mathbf{F}_{2}^{(m)}\right)^{\mathrm{T}}$

If equilibrium $\mathbf{z}^{(m) *}=\mathbf{z}^{\tau(m) *}$ of Eq. (152) (i.e., $\boldsymbol{f}^{(m)}\left(\mathbf{z}^{(m) *}\right.$, $\left.\left.\mathbf{z}^{\tau(m) *}\right)=\mathbf{0}\right)$ exists, then the approximate analytical solution of period-m flow of Eq. (135) exists in Eq. (147). In the vicinity of equilibrium $\mathbf{z}^{(m) *}=\mathbf{z}^{\tau(m) *}$, with $\mathbf{z}^{(m)}=\mathbf{z}^{(m) *}+\Delta \mathbf{z}^{(m)}$ and $\mathbf{z}^{\tau(m)}=\mathbf{z}^{\tau(m) *}+\Delta \mathbf{z}^{\tau(m)}$, the linearized equation of Eq. (152) is

$$
\begin{aligned}
\Delta \dot{\mathbf{z}}^{(m)}= & D_{\mathbf{z}^{(m)}} \mathbf{f}^{(m)}\left(\mathbf{z}^{(m) *}, \mathbf{z}^{\tau(m) *}\right) \Delta \mathbf{z}^{(m)} \\
& +D_{\mathbf{z}^{\tau(m)}} \mathbf{f}^{(m)}\left(\mathbf{z}^{(m) *}, \mathbf{z}^{\tau(m) *}\right) \Delta \mathbf{z}^{\tau(m)}
\end{aligned}
$$

and the eigenvalue analysis of equilibrium $\mathbf{z}^{(m) *}=\mathbf{z}^{\tau(m) *}$ is given by

$$
\begin{aligned}
& \mid D_{\mathbf{z}^{(m)}} \mathbf{f}^{(m)}\left(\mathbf{z}^{(m) *}, \mathbf{z}^{\tau(m) *}\right)-\lambda \mathbf{I}_{n(2 N+1) \times n(2 N+1)} \\
& \quad+D_{\mathbf{z}^{\tau(m)}} \mathbf{f}^{(m)}\left(\mathbf{z}^{(m) *}, \mathbf{z}^{\tau(m) *}\right) e^{-\lambda \tau} \mid=0
\end{aligned}
$$


where

$$
\begin{aligned}
& D_{\mathbf{z}^{(m)}} \mathbf{f}^{(m)}\left(\mathbf{z}^{(m) *}, \mathbf{z}^{\tau(m) *}\right) \\
& \quad=\partial \mathbf{f}^{(m)}\left(\mathbf{z}^{(m)}, \mathbf{z}^{\tau(m)}\right) /\left.\partial \mathbf{z}^{(m)}\right|_{\left(\mathbf{z}^{(m) *}, \mathbf{z}^{\tau(m) *}\right)}, \\
& D_{\mathbf{z}^{\tau(m)}} \mathbf{f}^{(m)}\left(\mathbf{z}^{(m) *}, \mathbf{z}^{\tau(m) *}\right) \\
& \quad=\partial \mathbf{f}^{(m)}\left(\mathbf{z}^{(m)}, \mathbf{z}^{\tau(m)}\right) /\left.\partial \mathbf{z}^{\tau(m)}\right|_{\left(\mathbf{z}^{(m) *}, \mathbf{z}^{\tau(m) *}\right)} .
\end{aligned}
$$

The stability and bifurcation of periodic solution can be classified by the eigenvalues of Eq. (154) at equilibrium $\mathbf{z}^{(m) *}=\mathbf{z}^{\tau(m) *}$ with

$\left(n_{1}, n_{2}, n_{3} \mid n_{4}, n_{5}, n_{6}\right)$

(i) If all eigenvalues of the equilibrium possess negative real parts, the approximate periodic solution is stable.

(ii) If at least one of eigenvalues of the equilibrium possesses positive real part, the approximate periodic solution is unstable.

(iii) The boundaries between stable and unstable equilibriums with higher order singularity give bifurcation and stability conditions with higher order singularity.

Proof The proof is similar to the proof of Theorem 10.

If $m \rightarrow \infty$ with $N \rightarrow \infty$, equation (147) will give the analytical expression of chaos in periodically forced, timedelayed, nonlinear dynamical systems in Eq. (135).

\section{Time-delayed, nonlinear vibration systems}

In this section, the analytical solutions of periodic motion in time-delayed, nonlinear vibration systems are presented because of extensively application in vibration control, and the local stability and bifurcation theory will be applied to determine the stability and bifurcation of approximate solutions of time-delayed, nonlinear vibration systems.

\subsection{Time-delayed, free vibration systems}

Periodic motions in time-delayed, nonlinear vibration systems will be discussed first. If such a time-delayed, vibration system has periodic motions with period $T=2 \pi / \Omega$, then such periodic motions can be expressed by the Fourier series.

Theorem 13 Consider a time-delayed, nonlinear vibration system as

$\ddot{\mathbf{x}}=\mathbf{f}\left(\mathbf{x}, \dot{\mathbf{x}}, \mathbf{x}^{\tau}, \dot{\mathbf{x}}^{\tau}, \mathbf{p}\right) \in \mathbf{R}^{n}$

where $\mathbf{f}\left(\mathbf{x}, \dot{\mathbf{x}}, \mathbf{x}^{\tau}, \dot{\mathbf{x}}^{\tau}, \mathbf{p}\right)$ is a $C^{r}$-continuous nonlinear function vector $(r \geq 1)$. If such dynamical system has a periodic motion $\mathbf{x}^{*}(t)$ with finite norm $\left\|\mathbf{x}^{*}\right\|$ and period $T=2 \pi / \Omega$, there is an analytical expression for the periodic motion of $\mathrm{Eq}$. (158) in the form of

$$
\begin{aligned}
\mathbf{x}^{*}= & \mathbf{a}_{0}(t)+\sum_{k=1}^{N} \mathbf{b}_{k}(t) \cos (k \Omega t)+\mathbf{c}_{k}(t) \sin (k \Omega t) \\
\mathbf{x}^{\tau *}= & \mathbf{a}_{0}^{\tau}(t)+\sum_{k=1}^{N} \mathbf{b}_{k}^{\tau}(t) \cos [k \Omega(t-\tau)] \\
& +\mathbf{c}_{k}^{\tau}(t) \sin [k \Omega(t-\tau)]
\end{aligned}
$$

with

$\mathbf{a}_{0}=\left(a_{01}, a_{02}, \ldots, a_{0 n}\right)^{\mathrm{T}}$,

$\mathbf{b}_{k}=\left(b_{k 1}, b_{k 2}, \ldots, b_{k n}\right)^{\mathrm{T}}$,

$\mathbf{c}_{k}=\left(c_{k 1}, c_{k 2}, \ldots, c_{k n}\right)^{\mathrm{T}}$;

$\mathbf{a}_{0}^{\tau}=\left(a_{01}^{\tau}, a_{02}^{\tau}, \ldots, a_{0 n}^{\tau}\right)^{\mathrm{T}}$,

$\mathbf{b}_{k}^{\tau}=\left(b_{k 1}^{\tau}, b_{k 2}^{\tau}, \ldots, b_{k n}^{\tau}\right)^{\mathrm{T}}$,

$\mathbf{c}_{k}^{\tau}=\left(c_{k 1}^{\tau}, c_{k 2}^{\tau}, \ldots, c_{k n}^{\tau}\right)^{\mathrm{T}}$;

and the corresponding coefficients slowly varying with time are determined by

$$
\begin{aligned}
& \ddot{\mathbf{a}}_{0}=\mathbf{F}_{0}\left(\mathbf{z}, \mathbf{z}_{1} ; \mathbf{z}^{\tau}, \mathbf{z}_{1}^{\tau}\right) \\
& \ddot{\mathbf{b}}=-2 \Omega \mathbf{k}_{1} \dot{\mathbf{c}}+\Omega^{2} \mathbf{k}_{2} \mathbf{b}+\mathbf{F}_{1}\left(\mathbf{z}, \mathbf{z}_{1} ; \mathbf{z}^{\tau}, \mathbf{z}_{1}^{\tau}\right), \\
& \ddot{\mathbf{c}}=2 \Omega \mathbf{k}_{1} \dot{\mathbf{b}}+\Omega^{2} \mathbf{k}_{2} \mathbf{c}+\mathbf{F}_{2}\left(\mathbf{z}, \mathbf{z}_{1} ; \mathbf{z}^{\tau}, \mathbf{z}_{1}^{\tau}\right)
\end{aligned}
$$

where for $N=1,2, \ldots, \infty$

$$
\begin{aligned}
& \mathbf{k}_{1}=\operatorname{diag}\left(\mathbf{I}_{n \times n}, 2 \mathbf{I}_{n \times n}, \ldots, N \mathbf{I}_{n \times n}\right), \\
& \mathbf{k}_{2}=\operatorname{diag}\left(\mathbf{I}_{n \times n}, 2^{2} \mathbf{I}_{n \times n}, \ldots, N^{2} \mathbf{I}_{n \times n}\right) ; \\
& \mathbf{b}=\left(\mathbf{b}_{1}, \mathbf{b}_{2}, \ldots, \mathbf{b}_{N}\right)^{\mathrm{T}}, \\
& \mathbf{c}=\left(\mathbf{c}_{1}, \mathbf{c}_{2}, \ldots, \mathbf{c}_{N}\right)^{\mathrm{T}} ; \\
& \mathbf{b}^{\tau}=\left(\mathbf{b}_{1}^{\tau}, \mathbf{b}_{2}^{\tau}, \ldots, \mathbf{b}_{N}^{\tau}\right)^{\mathrm{T}}, \\
& \mathbf{c}^{\tau}=\left(\mathbf{c}_{1}^{\tau}, \mathbf{c}_{2}^{\tau}, \ldots, \mathbf{c}_{N}^{\tau}\right)^{\mathrm{T}} ; \\
& \mathbf{F}_{1}=\left(\mathbf{F}_{11}, \mathbf{F}_{12}, \ldots, \mathbf{F}_{1 N}\right)^{\mathrm{T}}, \\
& \mathbf{F}_{2}=\left(\mathbf{F}_{21}, \mathbf{F}_{22}, \ldots, \mathbf{F}_{2 N}\right)^{\mathrm{T}} ; \\
& \mathbf{z}=\left(\mathbf{a}_{0}, \mathbf{b}, \mathbf{c}\right)^{\mathrm{T}}, \dot{\mathbf{z}}=\mathbf{z}_{1} ; \\
& \mathbf{z}^{\tau}=\left(\mathbf{a}_{0}^{\tau}, \mathbf{b}^{\tau}, \mathbf{c}^{\tau}\right)^{\mathrm{T}}, \dot{\mathbf{z}}^{\tau}=\mathbf{z}_{1}^{\tau}
\end{aligned}
$$

and for $k=1,2, \ldots, N$

$$
\begin{aligned}
& \mathbf{F}_{0}\left(\mathbf{z}, \mathbf{z}_{1} ; \mathbf{z}^{\tau}, \mathbf{z}_{1}^{\tau}\right) \\
&=\frac{1}{T} \int_{0}^{T} \mathbf{f}\left(\mathbf{x}^{*}, \dot{\mathbf{x}}^{*} ; \mathbf{x}^{\tau *}, \dot{\mathbf{x}}^{\tau *}, \mathbf{p}\right) d t ;
\end{aligned}
$$

$\mathbf{F}_{1 k}\left(\mathbf{z}, \mathbf{z}_{1} ; \mathbf{z}^{\tau}, \mathbf{z}_{1}^{\tau}\right)$

$$
=\frac{2}{T} \int_{0}^{T} \mathbf{f}\left(\mathbf{x}^{*}, \dot{\mathbf{x}}^{*} ; \mathbf{x}^{\tau *}, \dot{\mathbf{x}}^{\tau *}, \mathbf{p}\right) \cos (k \Omega t) d t,
$$


$\mathbf{F}_{2 k}\left(\mathbf{z}, \mathbf{z}_{1} ; \mathbf{z}^{\tau}, \mathbf{z}_{1}^{\tau}\right)$

$$
=\frac{2}{T} \int_{0}^{T} \mathbf{f}\left(\mathbf{x}^{*}, \dot{\mathbf{x}}^{*} ; \mathbf{x}^{\tau *}, \dot{\mathbf{x}}^{\tau *}, \mathbf{p}\right) \sin (k \Omega t) d t .
$$

The state-space form of Eq. (161) is

$\dot{\mathbf{z}}=\mathbf{z}_{1} \quad$ and $\quad \dot{\mathbf{z}}_{1}=\mathbf{g}\left(\mathbf{z}, \mathbf{z}_{1} ; \mathbf{z}^{\tau}, \mathbf{z}_{1}^{\tau}\right)$

where

$\mathbf{g}=\left(\mathbf{F}_{0},-2 \Omega \mathbf{k}_{1} \dot{\mathbf{c}}+\Omega^{2} \mathbf{k}_{2} \mathbf{b}+\mathbf{F}_{1}\right.$,

$$
\left.2 \Omega \mathbf{k}_{1} \dot{\mathbf{b}}+\Omega^{2} \mathbf{k}_{2} \mathbf{c}+\mathbf{F}_{2}\right)^{\mathrm{T}} \text {. }
$$

An equivalent system of Eq. (164) is

$\dot{\mathbf{y}}=\mathbf{f}\left(\mathbf{y}, \mathbf{y}^{\tau}\right)$

where

$\mathbf{y}=\left(\mathbf{z}, \mathbf{z}_{1}\right)^{\mathrm{T}}, \mathbf{y}^{\tau}=\left(\mathbf{z}^{\tau}, \mathbf{z}_{1}^{\tau}\right)^{\mathrm{T}}$ and $\mathbf{f}=\left(\mathbf{z}_{1}, \mathbf{g}\right)^{\mathrm{T}}$.

If equilibrium $\mathbf{y}^{*}=\mathbf{y}^{\tau *}$ of Eq. (166) (i.e., $\mathbf{f}\left(\mathbf{y}^{*}, \mathbf{y}^{\tau *}\right)=\mathbf{0}$ ) exists, then the analytical solution of periodic motion of Eq. (158) exists in Eq. (159). In vicinity of equilibrium $\mathbf{y}^{*}=\mathbf{y}^{\tau *}$, with $\mathbf{y}=\mathbf{y}^{*}+\Delta \mathbf{y}$ and $\mathbf{y}^{\tau}=\mathbf{y}^{\tau *}+\Delta \mathbf{y}^{\tau}$, the linearized equation of Eq. (166) is

$\Delta \dot{\mathbf{y}}=D_{\mathbf{y}} \mathbf{f}\left(\mathbf{y}^{*}, \mathbf{y}^{\tau *}\right) \Delta \mathbf{y}+D_{\mathbf{y}^{\tau}} \mathbf{f}\left(\mathbf{y}^{*}, \mathbf{y}^{\tau *}\right) \Delta \mathbf{y}^{\tau}$

and the eigenvalue analysis of equilibrium $\mathbf{y}^{*}=\mathbf{y}^{\tau *}$ is given by

$$
\begin{aligned}
& \mid D_{\mathbf{y}} \mathbf{f}\left(\mathbf{y}^{*}, \mathbf{y}^{\tau *}\right)-\lambda \mathbf{I}_{2 n(2 N+1) \times 2 n(2 N+1)} \\
& \quad+D_{\mathbf{y}^{\tau}} \mathbf{f}\left(\mathbf{y}^{*}, \mathbf{y}^{\tau *}\right) e^{-\lambda \tau} \mid=0
\end{aligned}
$$

where

$$
\begin{aligned}
& D_{\mathbf{y}} \mathbf{f}\left(\mathbf{y}^{*}, \mathbf{y}^{\tau *}\right)=\left.\frac{\partial \mathbf{f}\left(\mathbf{y}, \mathbf{y}^{\tau}\right)}{\partial \mathbf{y}}\right|_{\left(\mathbf{y}^{*}, \mathbf{y}^{\tau *}\right)}, \\
& D_{\mathbf{y}^{\tau}} \mathbf{f}\left(\mathbf{y}^{*}, \mathbf{y}^{\tau *}\right)=\left.\frac{\partial \mathbf{f}\left(\mathbf{y}, \mathbf{y}^{\tau}\right)}{\partial \mathbf{y}^{\tau}}\right|_{\left(\mathbf{y}^{*}, \mathbf{y}^{\tau *}\right)} .
\end{aligned}
$$

Thus, the stability and bifurcation of periodic solution can be classified by the eigenvalues of Eq. (168) at equilibrium $\mathbf{y}^{*}=\mathbf{y}^{\tau *}$ with

$\left(n_{1}, n_{2}, n_{3} \mid n_{4}, n_{5}, n_{6}\right)$

(i) If all eigenvalues of the equilibrium possess negative real parts, the approximate periodic solution is stable.

(ii) If at least one of eigenvalues of the equilibrium possesses positive real part, the approximate periodic solution is unstable.

(iii) The boundaries between stable and unstable equilibriums with higher order singularity give bifurcation and stability conditions with higher order singularity.

Proof Since $\mathbf{f}\left(\mathbf{x}, \dot{\mathbf{x}} ; \mathbf{x}^{\tau}, \dot{\mathbf{x}}^{\tau}, \mathbf{p}\right)$ is a $C^{r}$-continuous nonlinear function vector $(r \geq 1)$, the velocity $\dot{\mathbf{x}}$ should be $C^{r}$ continuous $(r \geq 1)$, and then the acceleration $\ddot{\mathbf{x}}$ should be bounded (i.e., ||$\ddot{\mathbf{x}}|| \leq K$ ). Thus, the Fourier series of periodic motion in Eq. (159) is convergent from the Fourier series theory. Taking derivatives of Eq. (159) with respect to time gives

$$
\begin{aligned}
\dot{\mathbf{x}}^{*}(t)= & \dot{\mathbf{a}}_{0}+\sum_{k=1}^{N}\left(\dot{\mathbf{b}}_{k}+k \Omega \mathbf{c}_{k}\right) \cos (k \Omega t) \\
& +\left(\dot{\mathbf{c}}_{k}-k \Omega \mathbf{b}_{k}\right) \sin (k \Omega t), \\
\ddot{\mathbf{x}}^{*}(t)= & \ddot{\mathbf{a}}_{0}+\sum_{k=1}^{N}\left(\ddot{\mathbf{b}}_{k}+2 k \Omega \dot{\mathbf{c}}_{k}-k^{2} \Omega^{2} \mathbf{b}_{k}\right) \cos (k \Omega t) \\
& +\left(\ddot{\mathbf{c}}_{k}-2 k \Omega \dot{\mathbf{b}}_{k}-k^{2} \Omega^{2} \mathbf{c}_{k}\right) \sin (k \Omega t) . \\
\dot{\mathbf{x}}^{* \tau}(t)= & \dot{\mathbf{a}}_{0}^{\tau}+\sum_{k=1}^{N}\left(\dot{\mathbf{b}}_{k}^{\tau}+k \Omega \mathbf{c}_{k}^{\tau}\right) \cos (k \Omega t) \\
& +\left(\dot{\mathbf{c}}_{k}^{\tau}-k \Omega \mathbf{b}_{k}^{\tau}\right) \sin (k \Omega t), \\
\ddot{\mathbf{x}}^{\tau *}(t)= & \ddot{\mathbf{a}}_{0}^{\tau}+\sum_{k=1}^{N}\left(\ddot{\mathbf{b}}_{k}^{\tau}+2 k \Omega \dot{\mathbf{c}}_{k}^{\tau}-k^{2} \Omega^{2} \mathbf{b}_{k}^{\tau}\right) \cos (k \Omega t) \\
& +\left(\ddot{\mathbf{c}}_{k}^{\tau}-2 k \Omega \dot{\mathbf{b}}_{k}^{\tau}-k^{2} \Omega^{2} \mathbf{c}_{k}^{\tau}\right) \sin (k \Omega t) .
\end{aligned}
$$

Since the coefficients vary slowly with time, substitution of the foregoing equation into Eq. (158) and averaging for constant, $\cos (k \Omega t)$ and $\sin (k \Omega t)(k=1,2, \ldots)$ yields for $k=1,2, \ldots, N$

$$
\begin{aligned}
& \ddot{\mathbf{a}}_{0}=\mathbf{F}_{0}\left(\mathbf{z}, \mathbf{z}_{1} ; \mathbf{z}^{\tau}, \mathbf{z}_{1}^{\tau}\right), \\
& \ddot{\mathbf{b}}_{k}=-2 \Omega k \dot{\mathbf{c}}_{k}+\Omega^{2} k \mathbf{b}_{k}+\mathbf{F}_{1 k}\left(\mathbf{z}, \mathbf{z}_{1} ; \mathbf{z}^{\tau}, \mathbf{z}_{1}^{\tau}\right), \\
& \ddot{\mathbf{c}}_{k}=2 \Omega k \dot{\mathbf{b}}_{k}+\Omega^{2} k \mathbf{c}_{k}+\mathbf{F}_{2 k}\left(\mathbf{z}, \mathbf{z}_{1} ; \mathbf{z}^{\tau}, \mathbf{z}_{1}^{\tau}\right) ;
\end{aligned}
$$

where

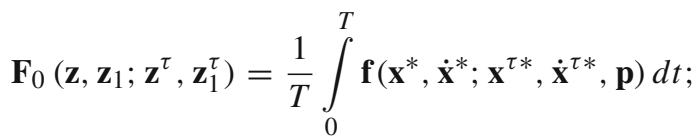

$\mathbf{F}_{1 k}\left(\mathbf{z}, \mathbf{z}_{1} ; \mathbf{z}^{\tau}, \mathbf{z}_{1}^{\tau}\right)=\frac{2}{T} \int_{0}^{T} \mathbf{f}\left(\mathbf{x}^{*}, \dot{\mathbf{x}}^{*} ; \mathbf{x}^{\tau *}, \dot{\mathbf{x}}^{\tau *}, \mathbf{p}\right) \cos (k \Omega t) d t$,

$\mathbf{F}_{2 k}\left(\mathbf{z}, \mathbf{z}_{1} ; \mathbf{z}^{\tau}, \mathbf{z}_{1}^{\tau}\right)=\frac{2}{T} \int_{0}^{T} \mathbf{f}\left(\mathbf{x}^{*}, \dot{\mathbf{x}}^{*} ; \mathbf{x}^{\tau *}, \dot{\mathbf{x}}^{\tau *}, \mathbf{p}\right) \sin (k \Omega t) d t$.

Introduce

$$
\begin{aligned}
& \mathbf{k}_{1}=\operatorname{diag}\left(\mathbf{I}_{n \times n}, 2 \mathbf{I}_{n \times n}, \ldots, N \mathbf{I}_{n \times n}\right), \\
& \mathbf{k}_{2}=\operatorname{diag}\left(\mathbf{I}_{n \times n}, 2^{2} \mathbf{I}_{n \times n}, \ldots, N^{2} \mathbf{I}_{n \times n}\right) \\
& \mathbf{b}=\left(\mathbf{b}_{1}, \mathbf{b}_{2}, \ldots, \mathbf{b}_{N}\right)^{\mathrm{T}}, \\
& \mathbf{c}=\left(\mathbf{c}_{1}, \mathbf{c}_{2}, \ldots, \mathbf{c}_{N}\right)^{\mathrm{T}} ; \\
& \mathbf{b}^{\tau}=\left(\mathbf{b}_{1}^{\tau}, \mathbf{b}_{2}^{\tau}, \ldots, \mathbf{b}_{N}^{\tau}\right)^{\mathrm{T}}, \\
& \mathbf{c}^{\tau}=\left(\mathbf{c}_{1}^{\tau}, \mathbf{c}_{2}^{\tau}, \ldots, \mathbf{c}_{N}^{\tau}\right)^{\mathrm{T}} ;
\end{aligned}
$$




$$
\begin{aligned}
& \mathbf{F}_{1}=\left(\mathbf{F}_{11}, \mathbf{F}_{12}, \ldots, \mathbf{F}_{1 N}\right)^{\mathrm{T}}, \\
& \mathbf{F}_{2}=\left(\mathbf{F}_{21}, \mathbf{F}_{22}, \ldots, \mathbf{F}_{2 N}\right)^{\mathrm{T}} \\
& \mathbf{z}=\left(\mathbf{a}_{0}, \mathbf{b}, \mathbf{c}\right)^{\mathrm{T}}, \dot{\mathbf{z}}=\mathbf{z}_{1} ; \\
& \mathbf{z}^{\tau}=\left(\mathbf{a}_{0}^{\tau}, \mathbf{b}^{\tau}, \mathbf{c}^{\tau}\right)^{\mathrm{T}}, \dot{\mathbf{z}}^{\tau}=\mathbf{z}_{1}^{\tau}
\end{aligned}
$$

Rearranging the foregoing equation gives Eq. (161), i.e.,

$$
\begin{aligned}
& \ddot{\mathbf{a}}_{0}=\mathbf{F}_{0}\left(\mathbf{z}, \mathbf{z}_{1} ; \mathbf{z}^{\tau}, \mathbf{z}_{1}^{\tau}\right), \\
& \ddot{\mathbf{b}}=-2 \Omega \mathbf{k}_{1} \dot{\mathbf{c}}+\Omega^{2} \mathbf{k}_{2} \mathbf{b}+\mathbf{F}_{1}\left(\mathbf{z}, \mathbf{z}_{1} ; \mathbf{z}^{\tau}, \mathbf{z}_{1}^{\tau}\right), \\
& \ddot{\mathbf{c}}=2 \Omega \mathbf{k}_{1} \dot{\mathbf{b}}+\Omega^{2} \mathbf{k}_{2} \mathbf{c}+\mathbf{F}_{2}\left(\mathbf{z}, \mathbf{z}_{1} ; \mathbf{z}^{\tau}, \mathbf{z}_{1}^{\tau}\right)
\end{aligned}
$$

Let

$\mathbf{z}=\left(\mathbf{a}_{0}, \mathbf{b}, \mathbf{c}\right)^{\mathrm{T}}, \mathbf{z}^{\tau}=\left(\mathbf{a}_{0}^{\tau}, \mathbf{b}^{\tau}, \mathbf{c}^{\tau}\right)^{\mathrm{T}} ; \dot{\mathbf{z}}=\mathbf{z}_{1}, \dot{\mathbf{z}}^{\tau}=\mathbf{z}_{1}^{\tau}$

$\mathbf{g}=\left(\mathbf{F}_{0},-2 \Omega \mathbf{k}_{1} \dot{\mathbf{c}}+\Omega^{2} \mathbf{k}_{2} \mathbf{b}+\mathbf{F}_{1}, 2 \Omega \mathbf{k}_{1} \dot{\mathbf{b}}+\Omega^{2} \mathbf{k}_{2} \mathbf{c}+\mathbf{F}_{2}\right)^{\mathrm{T}}$.

The equation in Eq. (161) becomes

$\dot{\mathbf{z}}=\mathbf{z}_{1} \quad$ and $\quad \dot{\mathbf{z}}_{1}=\mathbf{g}\left(\mathbf{z}, \mathbf{z}_{1} ; \mathbf{z}^{\tau}, \mathbf{z}_{1}^{\tau}\right)$

Letting $\mathbf{y}=\left(\mathbf{z}, \mathbf{z}_{1}\right)^{\mathrm{T}}, \mathbf{y}^{\tau}=\left(\mathbf{z}^{\tau}, \mathbf{z}_{1}^{\tau}\right)^{\mathrm{T}}$ and $\mathbf{f}=\left(\mathbf{z}_{1}, \mathbf{g}\right)^{\mathrm{T}}$, we have

$\dot{\mathbf{y}}=\mathbf{f}\left(\mathbf{y}, \mathbf{y}^{\tau}\right)$

Equilibrium $\mathbf{y}^{*}=\mathbf{y}^{\tau *}$ of the foregoing equation is determined by $\mathbf{f}\left(\mathbf{y}^{*}, \mathbf{y}^{\tau *}\right)=\mathbf{0}$, i.e.,

$\mathbf{0}=\mathbf{F}_{0}\left(\mathbf{a}_{0}^{*}, \mathbf{b}^{*}, \mathbf{c}^{*}, \mathbf{0}, \mathbf{0}, \mathbf{0} ; \mathbf{a}_{0}^{\tau *}, \mathbf{b}^{\tau *}, \mathbf{c}^{\tau *}, \mathbf{0}, \mathbf{0}, \mathbf{0}\right)$,

$\mathbf{0}=\Omega^{2} \mathbf{k}_{2} \mathbf{b}^{*}+\mathbf{F}_{1}\left(\mathbf{a}_{0}^{*}, \mathbf{b}^{*}, \mathbf{c}^{*}, \mathbf{0}, \mathbf{0}, \mathbf{0} ; \mathbf{a}_{0}^{\tau *}, \mathbf{b}^{\tau *}, \mathbf{c}^{\tau *}, \mathbf{0}, \mathbf{0}, \mathbf{0}\right)$,

$\mathbf{0}=\Omega^{2} \mathbf{k}_{2} \mathbf{c}^{*}+\mathbf{F}_{2}\left(\mathbf{a}_{0}^{*}, \mathbf{b}^{*}, \mathbf{c}^{*}, \mathbf{0}, \mathbf{0}, \mathbf{0} ; \mathbf{a}_{0}^{\tau *}, \mathbf{b}^{\tau *}, \mathbf{c}^{\tau *}, \mathbf{0}, \mathbf{0}, \mathbf{0}\right)$.

with

$\mathbf{a}_{0}^{(m) *}=\mathbf{a}_{0}^{\tau(m) *}, \quad \mathbf{b}^{(m) *}=\mathbf{b}^{\tau(m) *}, \quad \mathbf{c}^{(m) *}=\mathbf{c}^{\tau(m) *}$.

Thus, solutions of the forgoing equation are the exisitance conditions of periodic solutions for time-delayed, nonlinear vibration systems. If the foregoing equation gives equilibriums $\mathbf{y}^{*}=\mathbf{y}^{\tau *}$, in vicinity of $\mathbf{y}^{*}=\mathbf{y}^{\tau *}$, with $\mathbf{y}=\mathbf{y}^{*}+\Delta \mathbf{y}$ and $\mathbf{y}^{\tau}=\mathbf{y}^{\tau *}+\Delta \mathbf{y}^{\tau}$, the linearized equation of $\dot{\mathbf{y}}=\mathbf{f}\left(\mathbf{y}, \mathbf{y}^{\tau}\right)$ is

$$
\Delta \dot{\mathbf{y}}=D_{\mathbf{y}} \mathbf{f}\left(\mathbf{y}^{*}, \mathbf{y}^{\tau *}\right) \Delta \mathbf{y}+D_{\mathbf{y}^{\tau}} \mathbf{f}\left(\mathbf{y}^{*}, \mathbf{y}^{\tau *}\right) \Delta \mathbf{y}^{\tau}
$$

and the eigenvalue analysis of equilibrium $\mathbf{y}^{*}=\mathbf{y}^{\tau *}$ is given by

$$
\begin{aligned}
& \mid D_{\mathbf{y}} \mathbf{f}\left(\mathbf{y}^{*}, \mathbf{y}^{\tau *}\right)-\lambda \mathbf{I}_{2 n(2 N+1) \times 2 n(2 N+1)} \\
& \quad+D_{\mathbf{y}^{\tau}} \mathbf{f}\left(\mathbf{y}^{*}, \mathbf{y}^{\tau *}\right) e^{-\lambda \tau} \mid=0
\end{aligned}
$$

where

$$
\begin{aligned}
D_{\mathbf{y}} \mathbf{f}\left(\mathbf{y}^{*}, \mathbf{y}^{\tau *}\right) & =\left.\frac{\partial \mathbf{f}\left(\mathbf{y}, \mathbf{y}^{\tau}\right)}{\partial \mathbf{y}}\right|_{\left(\mathbf{y}^{*}, \mathbf{y}^{\tau *}\right)}, \\
D_{\mathbf{y}^{\tau}} \mathbf{f}\left(\mathbf{y}^{*}, \mathbf{y}^{\tau *}\right) & =\left.\frac{\partial \mathbf{f}\left(\mathbf{y}, \mathbf{y}^{\tau}\right)}{\partial \mathbf{y}^{\tau}}\right|_{\left(\mathbf{y}^{*}, \mathbf{y}^{\tau *}\right)} .
\end{aligned}
$$

Thus, the stability and bifurcation of periodic solution can be classified by the eigenvalues of Eq. (168) at equilibrium $\mathbf{y}^{*}=\mathbf{y}^{\tau *}$ with

$\left(n_{1}, n_{2}, n_{3} \mid n_{4}, n_{5}, n_{6}\right)$.

From the stability and bifurcation theory of dynamical systems at equilibrium, the stability and bifurcation of the periodic solutions can be classified as stated in the theorem. This theorem is proved.

If the Hopf bifurcation of equilibriums of Eq. (161) occurs, there is a periodic solution of coefficients in Eq. (160) with a frequency $\omega$. As discussed from Eq. (119) to Eq. (123)), there is a period- $m$ flow as in Eq. (124). If $m \omega \neq k \Omega$ for any $m$ and $k$, the solutions will be quasi-periodic or chaotic instead of periodic. Herein, period- $m$ motion in time-delayed, nonlinear vibration systems will be discussed only.

Theorem 14 Consider a time-delayed, nonlinear vibration system in Eq. (158). If such a time-delayed, vibration system has a period-m motion $\mathbf{x}^{(m) *}(t)$ with finite norm $\left\|\mathbf{x}^{(m) *}\right\|$ and period $T=2 \pi / \Omega$, there is an analytical expression for the periodic flow of Eq. (158) in the form of

$$
\begin{aligned}
\mathbf{x}^{(m) *}(t)= & \mathbf{a}_{0}^{(m)}(t)+\sum_{k=1}^{N} \mathbf{b}_{k / m}(t) \cos \left(\frac{k}{m} \Omega t\right) \\
& +\mathbf{c}_{k / m}(t) \sin \left(\frac{k}{m} \Omega t\right) ; \\
\mathbf{x}^{(m) \tau *}(t)= & \mathbf{a}_{0}^{\tau(m)}(t)+\sum_{k=1}^{N} \mathbf{b}_{k / m}^{\tau}(t) \cos \left[\frac{k}{m} \Omega(t-\tau)\right] \\
& +\mathbf{c}_{k / m}^{\tau}(t) \sin \left[\frac{k}{m} \Omega(t-\tau)\right]
\end{aligned}
$$

with for $k=1,2, \ldots, N$

$\mathbf{a}_{0}^{(m)}=\left(a_{01}^{(m)}, a_{02}^{(m)}, \ldots, a_{0 n}^{(m)}\right)^{\mathrm{T}}$,

$\mathbf{b}_{k / m}=\left(b_{k / m 1}, b_{k / m 2}, \ldots, b_{k / m n}\right)^{\mathrm{T}}$,

$\mathbf{c}_{k / m}=\left(c_{k / m 1}, c_{k / m 2}, \ldots, c_{k / m n}\right)^{\mathrm{T}}$,

$\mathbf{a}_{0}^{\tau(m)}=\left(a_{01}^{\tau(m)}, a_{02}^{\tau(m)}, \ldots, a_{0 n}^{\tau(m)}\right)^{\mathrm{T}}$,

$\mathbf{b}_{k / m}^{\tau}=\left(b_{k / m 1}^{\tau}, b_{k / m 2}^{\tau}, \ldots, b_{k / m n}^{\tau}\right)^{\mathrm{T}}$,

$\mathbf{c}_{k / m}^{\tau}=\left(c_{k / m 1}^{\tau}, c_{k / m 2}^{\tau}, \ldots, c_{k / m n}^{\tau}\right)^{\mathrm{T}}$.

and the corresponding coefficients slowly varying with time are determined by

$$
\begin{aligned}
\ddot{\mathbf{a}}_{0}^{(m)}= & \mathbf{F}_{0}^{(m)}\left(\mathbf{z}^{(m)}, \mathbf{z}_{1}^{(m)} ; \mathbf{z}^{\tau(m)}, \mathbf{z}_{1}^{\tau(m)}\right), \\
\ddot{\mathbf{b}}^{(m)}= & -2 \frac{\Omega}{m} \mathbf{k}_{1} \dot{\mathbf{c}}^{(m)}+\frac{\Omega^{2}}{m^{2}} \mathbf{k}_{2} \mathbf{b}^{(m)} \\
& +\mathbf{F}_{1}^{(m)}\left(\mathbf{z}^{(m)}, \mathbf{z}_{1}^{(m)} ; \mathbf{z}^{\tau(m)}, \mathbf{z}_{1}^{\tau(m)}\right), \\
\ddot{\mathbf{c}}^{(m)}= & 2 \frac{\Omega}{m} \mathbf{k}_{1} \dot{\mathbf{b}}^{(m)}+\frac{\Omega^{2}}{m^{2}} \mathbf{k}_{2} \mathbf{c}^{(m)} \\
& +\mathbf{F}_{2}^{(m)}\left(\mathbf{z}^{(m)}, \mathbf{z}_{1}^{(m)} ; \mathbf{z}^{\tau(m)}, \mathbf{z}_{1}^{\tau(m)}\right)
\end{aligned}
$$


where for $N=1,2, \ldots, \infty$

$$
\begin{aligned}
& \mathbf{k}_{1}=\operatorname{diag}\left(\mathbf{I}_{n \times n}, 2 \mathbf{I}_{n \times n}, \ldots, N \mathbf{I}_{n \times n}\right) \text {, } \\
& \mathbf{k}_{2}=\operatorname{diag}\left(\mathbf{I}_{n \times n}, 2^{2} \mathbf{I}_{n \times n}, \ldots, N^{2} \mathbf{I}_{n \times n}\right) \text {, } \\
& \mathbf{b}^{(m)}=\left(\mathbf{b}_{1 / m}, \mathbf{b}_{2 / m}, \ldots, \mathbf{b}_{N / m}\right)^{\mathrm{T}} \text {, } \\
& \mathbf{c}^{(m)}=\left(\mathbf{c}_{1 / m}, \mathbf{c}_{2 / m}, \ldots, \mathbf{c}_{N / m}\right)^{\mathrm{T}} \text {, } \\
& \mathbf{b}^{\tau(m)}=\left(\mathbf{b}_{1 / m}^{\tau}, \mathbf{b}_{2 / m}^{\tau}, \ldots, \mathbf{b}_{N / m}^{\tau}\right)^{\mathrm{T}}, \\
& \mathbf{c}^{\tau(m)}=\left(\mathbf{c}_{1 / m}^{\tau}, \mathbf{c}_{2 / m}^{\tau}, \ldots, \mathbf{c}_{N / m}^{\tau}\right)^{\mathrm{T}} \text {, } \\
& \mathbf{F}_{1}^{(m)}=\left(\mathbf{F}_{11}^{(m)}, \mathbf{F}_{12}^{(m)}, \ldots, \mathbf{F}_{1 N}^{(m)}\right)^{\mathrm{T}}, \\
& \mathbf{F}_{2}^{(m)}=\left(\mathbf{F}_{21}^{(m)}, \mathbf{F}_{22}^{(m)}, \ldots, \mathbf{F}_{2 N}^{(m)}\right)^{\mathrm{T}} \text {; } \\
& \mathbf{z}^{(m)}=\left(\mathbf{a}_{0}^{(m)}, \mathbf{b}^{(m)}, \mathbf{c}^{(m)}\right)^{\mathrm{T}}, \dot{\mathbf{z}}^{(m)}=\mathbf{z}_{1}^{(m)}, \\
& \mathbf{z}^{\tau(m)}=\left(\mathbf{a}_{0}^{\tau(m)}, \mathbf{b}^{\tau(m)}, \mathbf{c}^{\tau(m)}\right)^{\mathrm{T}}, \dot{\mathbf{z}}^{\tau(m)}=\mathbf{z}_{1}^{\tau(m)} \\
& =\frac{1}{m T} \int_{0}^{m T} \mathbf{f}\left(\mathbf{x}^{(m) *}, \dot{\mathbf{x}}^{(m) *}, \mathbf{x}^{\tau(m) *}, \dot{\mathbf{x}}^{\tau(m) *}, \mathbf{p}\right) d t ; \\
& \mathbf{F}_{1 k}^{(m)}\left(\mathbf{z}^{(m)}, \mathbf{z}_{1}^{(m)} ; \mathbf{z}^{\tau(m)}, \mathbf{z}_{1}^{\tau(m)}\right) \\
& =\frac{2}{m T} \int_{0}^{m T} \mathbf{f}\left(\mathbf{x}^{(m) *}, \dot{\mathbf{x}}^{(m) *}, \mathbf{x}^{\tau(m) *}, \dot{\mathbf{x}}^{\tau(m) *}, \mathbf{p}\right) \cos \left(\frac{k}{m} \Omega t\right) d t, \\
& \mathbf{F}_{2 k}^{(m)}\left(\mathbf{z}^{(m)}, \mathbf{z}_{1}^{(m)} ; \mathbf{z}^{\tau(m)}, \mathbf{z}_{1}^{\tau(m)}\right) \\
& =\frac{2}{m T} \int_{0}^{m T} \mathbf{f}\left(\mathbf{x}^{(m) *}, \dot{\mathbf{x}}^{(m) *}, \mathbf{x}^{\tau(m) *}, \dot{\mathbf{x}}^{\tau(m) *}, \mathbf{p}\right) \sin \left(\frac{k}{m} \Omega t\right) d t \text {. }
\end{aligned}
$$

The state-space form of Eq. (174) is

$\dot{\mathbf{z}}^{(m)}=\mathbf{z}_{1}^{(m)}$ and $\dot{\mathbf{z}}_{1}^{(m)}=\mathbf{g}^{(m)}\left(\mathbf{z}^{(m)}, \mathbf{z}_{1}^{(m)} ; \mathbf{z}^{\tau(m)}, \mathbf{z}_{1}^{\tau(m)}\right)$

where

$$
\begin{aligned}
\mathbf{g}^{(m)}= & \left(\mathbf{F}_{0}^{(m)},-2 \frac{\Omega}{m} \mathbf{k}_{1} \dot{\mathbf{c}}^{(m)}+\frac{\Omega^{2}}{m^{2}} \mathbf{k}_{2} \mathbf{b}^{(m)}+\mathbf{F}_{1}^{(m)},\right. \\
& \left.2 \frac{\Omega}{m} \mathbf{k}_{1} \dot{\mathbf{b}}^{(m)}+\frac{\Omega^{2}}{m^{2}} \mathbf{k}_{2} \mathbf{c}^{(m)}+\mathbf{F}_{2}^{(m)}\right)^{\mathrm{T}} .
\end{aligned}
$$

An equivalent system of Eq. (177) is

$\dot{\mathbf{y}}^{(m)}=\mathbf{f}^{(m)}\left(\mathbf{y}^{(m)}, \mathbf{y}^{\tau(m)}\right)$

where

$\mathbf{y}^{(m)}=\left(\mathbf{z}^{(m)}, \mathbf{z}_{1}^{(m)}\right)^{\mathrm{T}}, \mathbf{y}^{\tau(m)}=\left(\mathbf{z}^{\tau(m)}, \mathbf{z}_{1}^{\tau(m)}\right)^{\mathrm{T}}$ and

$\mathbf{f}^{(m)}=\left(\mathbf{z}_{1}^{(m)}, \mathbf{g}^{(m)}\right)^{\mathrm{T}}$.

If equilibrium $\mathbf{y}^{(m) *}=\mathbf{y}^{\tau(m) *}$ of Eq. (179) (i.e. $\mathbf{f}^{(m)}\left(\mathbf{y}^{(m) *}\right.$, $\left.\left.\mathbf{y}^{\tau(m) *}\right)=\mathbf{0}\right)$ exists, then the analytical solution of period$m$ motion of Eq. (158) exists in Eq. (172). In vicinity of equilibrium $\mathbf{y}^{(m) *}=\mathbf{y}^{\tau(m) *}$, with $\mathbf{y}^{(m)}=\mathbf{y}^{(m) *}+\Delta \mathbf{y}^{(m)}$ and $\mathbf{y}^{\tau(m)}=\mathbf{y}^{\tau(m) *}+\Delta \mathbf{y}^{\tau(m)}$, the linearized equation of Eq. (179) is

$$
\begin{gathered}
\Delta \dot{\mathbf{y}}^{(m)}=D_{\mathbf{y}^{(m)}} \mathbf{f}^{(m)}\left(\mathbf{y}^{(m) *}, \mathbf{y}^{\tau(m) *}\right) \Delta \mathbf{y}^{(m)} \\
\quad+D_{\mathbf{y}^{\tau(m)}} \mathbf{f}^{(m)}\left(\mathbf{y}^{(m) *}, \mathbf{y}^{\tau(m) *}\right) \Delta \mathbf{y}^{\tau(m)}
\end{gathered}
$$

and the eigenvalue analysis of the equilibrium $\mathbf{y}^{*}$ is given by

$$
\begin{aligned}
& \mid D_{\mathbf{y}^{(m)}} \mathbf{f}^{(m)}\left(\mathbf{y}^{(m) *}, \mathbf{y}^{\tau(m) *}\right)-\lambda \mathbf{I}_{2 n(2 N+1) \times 2 n(2 N+1)} \\
& \quad+D_{\mathbf{y}^{\tau(m)}} \mathbf{f}^{(m)}\left(\mathbf{y}^{(m) *}, \mathbf{y}^{\tau(m) *}\right) e^{-\lambda \tau} \mid=0
\end{aligned}
$$

where

$$
\begin{aligned}
& D_{\mathbf{y}^{(m)}} \mathbf{f}^{(m)}\left(\mathbf{y}^{(m) *}, \mathbf{y}^{\tau(m) *}\right) \\
& \quad=\partial \mathbf{f}^{(m)}\left(\mathbf{y}^{(m)}, \mathbf{y}^{\tau(m)}\right) /\left.\partial \mathbf{y}^{(m)}\right|_{\left(\mathbf{y}^{(m) *}, \mathbf{y}^{\tau(m) *)}\right.}, \\
& D_{\mathbf{y}^{\tau(m)}} \mathbf{f}^{(m)}\left(\mathbf{y}^{(m) *}, \mathbf{y}^{\tau(m) *}\right) \\
& \quad=\partial \mathbf{f}^{(m)}\left(\mathbf{y}^{(m)}, \mathbf{y}^{\tau(m)}\right) /\left.\partial \mathbf{y}^{\tau(m)}\right|_{\left(\mathbf{y}^{(m) *}, \mathbf{y}^{\tau(m) *)}\right.} .
\end{aligned}
$$

Thus, the stability and bifurcation of periodic solution can be classified by the eigenvalues of Eq. (181) at equilibrium $\mathbf{y}^{(m) *}=\mathbf{y}^{\tau(m) *}$ with

$\left(n_{1}, n_{2}, n_{3} \mid n_{4}, n_{5}, n_{6}\right)$.

(i) If all eigenvalues of the equilibrium possess negative real parts, the periodic solution is stable.

(ii) If at least one of eigenvalues of the equilibrium possesses positive real part, the approximate steady-state solution is unstable.

(iii) The boundary between the stable and unstable equilibriums with higher order singularity gives the bifurcation conditions and stability with higher order singularity.

Proof The proof is similar to the proof of Theorem 13. Since $\mathbf{f}\left(\mathbf{x}, \dot{\mathbf{x}} ; \mathbf{x}^{\tau}, \dot{\mathbf{x}}^{\tau}, \mathbf{p}\right)$ is a $C^{r}$-continuous nonlinear function vector $(r \geq 1)$, velocity $\dot{\mathbf{x}}$ should be $C^{r}$-continuous $(r \geq 1)$, and then acceleration $\ddot{\mathbf{x}}$ should be bounded (i.e., ||$\ddot{\mathbf{x}} \|<K$ ). Thus, the Fourier series expression of periodic motion in Eq. (172) is convergent from the Fourier series theory. Taking derivatives of Eq. (172) gives

$$
\begin{aligned}
\dot{\mathbf{x}}^{(m) *}(t)= & \dot{\mathbf{a}}_{0}^{(m)}+\sum_{k=1}^{N}\left(\dot{\mathbf{b}}_{k / m}+\frac{k}{m} \Omega \mathbf{c}_{k / m}\right) \cos \left(\frac{k}{m} \Omega t\right) \\
& +\left(\dot{\mathbf{c}}_{k / m}-\frac{k}{m} \Omega \mathbf{b}_{k / m}\right) \sin \left(\frac{k}{m} \Omega t\right), \\
\ddot{\mathbf{x}}^{(m) *}(t)= & \ddot{\mathbf{a}}_{0}^{(m)}+\sum_{k=1}^{N}\left(\ddot{\mathbf{b}}_{k / m}+2 \frac{k}{m} \Omega \dot{\mathbf{c}}_{k / m}-\frac{k^{2}}{m^{2}} \Omega^{2} \mathbf{b}_{k / m}\right) \\
& \times \cos \left(\frac{k}{m} \Omega t\right)+\left(\ddot{\mathbf{c}}_{k / m}-2 \frac{k}{m} \Omega \dot{\mathbf{b}}_{k / m}\right. \\
& \left.-\frac{k^{2}}{m^{2}} \Omega^{2} \mathbf{c}_{k / m}\right) \sin \left(\frac{k}{m} \Omega t\right) .
\end{aligned}
$$




$$
\begin{aligned}
\dot{\mathbf{x}}^{(m) \tau *}(t)= & \dot{\mathbf{a}}_{0}^{\tau(m)}+\sum_{k=1}^{N}\left(\dot{\mathbf{b}}_{k / m}^{\tau}+\frac{k}{m} \Omega \mathbf{c}_{k / m}^{\tau}\right) \cos \left[\frac{k}{m} \Omega(t-\tau)\right] \\
& +\left(\dot{\mathbf{c}}_{k / m}^{\tau}-\frac{k}{m} \Omega \mathbf{b}_{k / m}^{\tau}\right) \sin \left[\frac{k}{m} \Omega(t-\tau)\right], \\
\ddot{\mathbf{x}}^{(m) \tau *}(t)= & \ddot{\mathbf{a}}_{0}^{\tau(m)}+\sum_{k=1}^{N}\left(\ddot{\mathbf{b}}_{k / m}^{\tau}+2 \frac{k}{m} \Omega \dot{\mathbf{c}}_{k / m}^{\tau}-\frac{k^{2}}{m^{2}} \Omega^{2} \mathbf{b}_{k / m}^{\tau}\right) \\
& \times \cos \left[\frac{k}{m} \Omega(t-\tau)\right]+\left(\ddot{\mathbf{c}}_{k / m}^{\tau}-2 \frac{k}{m} \Omega \dot{\mathbf{b}}_{k / m}^{\tau}\right. \\
& \left.-\frac{k^{2}}{m^{2}} \Omega^{2} \mathbf{c}_{k / m}^{\tau}\right) \sin \left[\frac{k}{m} \Omega(t-\tau)\right] .
\end{aligned}
$$

Since the coefficients vary slowly with time, substitution of the foregoing equation into Eq. (158) and averaging for constants, $\cos (k \Omega t / m)$ and $\sin (k \Omega t / m)(k=1,2, \ldots)$ yields for $k=1,2, \ldots, N$

$$
\begin{aligned}
\ddot{\mathbf{a}}_{0}^{(m)}= & \mathbf{F}_{0}^{(m)}\left(\mathbf{z}^{(m)}, \mathbf{z}_{1}^{(m)} ; \mathbf{z}^{\tau(m)}, \mathbf{z}_{1}^{\tau(m)}\right), \\
\ddot{\mathbf{b}}_{k / m}= & -2 \frac{\Omega}{m} k \dot{\mathbf{c}}_{k / m}+\frac{\Omega^{2}}{m^{2}} k^{2} \mathbf{b}_{k / m} \\
& +\mathbf{F}_{1 k}^{(m)}\left(\mathbf{z}^{(m)}, \mathbf{z}_{1}^{(m)} ; \mathbf{z}^{\tau(m)}, \mathbf{z}_{1}^{\tau(m)}\right), \\
\ddot{\mathbf{c}}_{k / m}= & 2 \frac{\Omega}{m} k \dot{\mathbf{b}}_{k / m}+\frac{\Omega^{2}}{m^{2}} k^{2} \mathbf{c}_{k / m} \\
& +\mathbf{F}_{2 k}^{(m)}\left(\mathbf{z}^{(m)}, \mathbf{z}_{1}^{(m)} ; \mathbf{z}^{\tau(m)}, \mathbf{z}_{1}^{\tau(m)}\right)
\end{aligned}
$$

where for $k=1,2, \ldots, N$

$$
\begin{aligned}
& \mathbf{F}_{0}^{(m)}\left(\mathbf{z}^{(m)}, \mathbf{z}_{1}^{(m)} ; \mathbf{z}^{\tau(m)}, \mathbf{z}_{1}^{\tau(m)}\right) \\
& =\frac{1}{m T} \int_{0}^{m T} \mathbf{f}\left(\mathbf{x}^{(m) *}, \dot{\mathbf{x}}^{(m) *} ; \mathbf{x}^{\tau(m) *}, \dot{\mathbf{x}}^{\tau(m) *}, \mathbf{p}\right) d t ; \\
& \mathbf{F}_{1 k}^{(m)}\left(\mathbf{z}^{(m)}, \mathbf{z}_{1}^{(m)} ; \mathbf{z}^{\tau(m)}, \mathbf{z}_{1}^{\tau(m)}\right) \\
& =\frac{2}{m T} \int_{0}^{m T} \mathbf{f}\left(\mathbf{x}^{(m) *}, \dot{\mathbf{x}}^{(m) *} ; \mathbf{x}^{\tau(m) *}, \dot{\mathbf{x}}^{\tau(m) *}, \mathbf{p}\right) \cos \left(\frac{k}{m} \Omega t\right) d t, \\
& \mathbf{F}_{2 k}^{(m)}\left(\mathbf{z}^{(m)}, \mathbf{z}_{1}^{(m)} ; \mathbf{z}^{\tau(m)}, \mathbf{z}_{1}^{\tau(m)}\right) \\
& =\frac{2}{m T} \int_{0}^{m T} \mathbf{f}\left(\mathbf{x}^{(m) *}, \dot{\mathbf{x}}^{(m) *} ; \mathbf{x}^{\tau(m) *}, \dot{\mathbf{x}}^{\tau(m) *}, \mathbf{p}\right) \sin \left(\frac{k}{m} \Omega t\right) d t ;
\end{aligned}
$$

and

$$
\begin{aligned}
& \mathbf{k}_{1}=\operatorname{diag}\left(\mathbf{I}_{n \times n}, 2 \mathbf{I}_{n \times n}, \ldots, N \mathbf{I}_{n \times n}\right), \\
& \mathbf{k}_{2}=\operatorname{diag}\left(\mathbf{I}_{n \times n}, 2^{2} \mathbf{I}_{n \times n}, \ldots, N^{2} \mathbf{I}_{n \times n}\right) ; \\
& \mathbf{b}^{(m)}=\left(\mathbf{b}_{1 / m}, \mathbf{b}_{2 / m}, \ldots, \mathbf{b}_{N / m}\right)^{\mathrm{T}}, \\
& \mathbf{c}^{(m)}=\left(\mathbf{c}_{1 / m}, \mathbf{c}_{2 / m}, \ldots, \mathbf{c}_{N / m}\right)^{\mathrm{T}} ; \\
& \mathbf{b}^{\tau(m)}=\left(\mathbf{b}_{1 / m}^{\tau}, \mathbf{b}_{2 / m}^{\tau}, \ldots, \mathbf{b}_{N / m}^{\tau}\right)^{\mathrm{T}}, \\
& \mathbf{c}^{\tau(m)}=\left(\mathbf{c}_{1 / m}^{\tau}, \mathbf{c}_{2 / m}^{\tau}, \ldots, \mathbf{c}_{N / m}^{\tau}\right)^{\mathrm{T}} ; \\
& \mathbf{F}_{1}^{(m)}=\left(\mathbf{F}_{11}^{(m)}, \mathbf{F}_{12}^{(m)}, \ldots, \mathbf{F}_{1 N}^{(m)}\right)^{\mathrm{T}}, \\
& \mathbf{F}_{2}^{(m)}=\left(\mathbf{F}_{21}^{(m)}, \mathbf{F}_{22}^{(m)}, \ldots, \mathbf{F}_{2 N}^{(m)}\right)^{\mathrm{T}} ;
\end{aligned}
$$

$\mathbf{z}^{(m)}=\left(\mathbf{a}_{0}^{(m)}, \mathbf{b}^{(m)}, \mathbf{c}^{(m)}\right)^{\mathrm{T}}, \dot{\mathbf{z}}^{(m)}=\mathbf{z}_{1}^{(m)} ;$

$\mathbf{z}^{\tau(m)}=\left(\mathbf{a}_{0}^{\tau(m)}, \mathbf{b}^{\tau(m)}, \mathbf{c}^{\tau(m)}\right)^{\mathrm{T}}, \dot{\mathbf{z}}^{\tau(m)}=\mathbf{z}_{1}^{\tau(m)}$

for $N=1,2, \ldots, \infty$.

Rearranging the foregoing equation gives Eq. (174), i.e.,

$$
\begin{aligned}
\ddot{\mathbf{a}}_{0}^{(m)}= & \mathbf{F}_{0}^{(m)}\left(\mathbf{z}^{(m)}, \mathbf{z}_{1}^{(m)} ; \mathbf{z}^{\tau(m)}, \mathbf{z}_{1}^{\tau(m)}\right), \\
\ddot{\mathbf{b}}^{(m)}=- & -2 \frac{\Omega}{m} \mathbf{k}_{1} \dot{\mathbf{c}}^{(m)}+\frac{\Omega^{2}}{m^{2}} \mathbf{k}_{2} \mathbf{b}^{(m)} \\
& +\mathbf{F}_{1}^{(m)}\left(\mathbf{z}^{(m)}, \mathbf{z}_{1}^{(m)} ; \mathbf{z}^{\tau(m)}, \mathbf{z}_{1}^{\tau(m)}\right), \\
\ddot{\mathbf{c}}^{(m)}= & 2 \frac{\Omega}{m} \mathbf{k}_{1} \dot{\mathbf{b}}^{(m)}+\frac{\Omega^{2}}{m^{2}} \mathbf{k}_{2} \mathbf{c}^{(m)} \\
& +\mathbf{F}_{2}^{(m)}\left(\mathbf{z}^{(m)}, \mathbf{z}_{1}^{(m)} ; \mathbf{z}^{\tau(m)}, \mathbf{z}_{1}^{\tau(m)}\right)
\end{aligned}
$$

Introduce

$$
\begin{aligned}
\mathbf{g}^{(m)}= & \left(\mathbf{F}_{0}^{(m)},-2 \frac{\Omega}{m} \mathbf{k}_{1} \dot{\mathbf{c}}^{(m)}+\frac{\Omega^{2}}{m^{2}} \mathbf{k}_{2} \mathbf{b}^{(m)}+\mathbf{F}_{1}^{(m)},\right. \\
& \left.2 \frac{\Omega}{m} \mathbf{k}_{1} \dot{\mathbf{b}}^{(m)}+\frac{\Omega^{2}}{m^{2}} \mathbf{k}_{2} \mathbf{c}^{(m)}+\mathbf{F}_{2}^{(m)}\right) \mathrm{T}
\end{aligned}
$$

The equation in Eq. (174) becomes

$\dot{\mathbf{z}}^{(m)}=\mathbf{z}_{1}^{(m)} \quad$ and $\quad \dot{\mathbf{z}}_{1}^{(m)}=\mathbf{g}^{(m)}\left(\mathbf{z}^{(m)}, \mathbf{z}_{1}^{(m)} ; \mathbf{z}^{\tau(m)}, \mathbf{z}_{1}^{\tau(m)}\right)$.

Letting

$\mathbf{y}^{(m)}=\left(\mathbf{z}^{(m)}, \mathbf{z}_{1}^{(m)}\right)^{\mathrm{T}}$,

$\mathbf{y}^{\tau(m)}=\left(\mathbf{z}^{\tau(m)}, \mathbf{z}_{1}^{\tau(m)}\right)^{\mathrm{T}}, \mathbf{f}^{(m)}=\left(\mathbf{z}_{1}^{(m)}, \mathbf{g}^{(m)}\right)^{\mathrm{T}}$

we have

$\dot{\mathbf{y}}^{(m)}=\mathbf{f}^{(m)}\left(\mathbf{y}^{(m)}, \mathbf{y}^{\tau(m)}\right)$.

Equilibrium of the foregoing equation is determined by $\left.\mathbf{f}^{(m)}\left(\mathbf{y}^{(m) *}, \mathbf{y}^{\tau(m) *}\right)=\mathbf{0}\right)$, i.e.,

$\mathbf{0}=\mathbf{F}_{0}\left(\mathbf{a}_{0}^{(m) *}, \mathbf{b}^{(m) *}, \mathbf{c}^{(m) *}, \mathbf{0}, \mathbf{0}, \mathbf{0} ;\right.$

$$
\begin{aligned}
& \left.\mathbf{a}_{0}^{\tau(m) *}, \mathbf{b}^{\tau(m) *}, \mathbf{c}^{\tau(m) *}, \mathbf{0}, \mathbf{0}, \mathbf{0}\right), \\
\mathbf{0}= & \frac{1}{m^{2}} \Omega^{2} \mathbf{k}_{2} \mathbf{b}^{(m) *}+\mathbf{F}_{1}\left(\mathbf{a}_{0}^{(m) *}, \mathbf{b}^{(m) *}, \mathbf{c}^{(m) *}, \mathbf{0}, \mathbf{0}, \mathbf{0} ;\right. \\
& \left.\mathbf{a}_{0}^{\tau(m) *}, \mathbf{b}^{\tau(m) *}, \mathbf{c}^{\tau(m) *}, \mathbf{0}, \mathbf{0}, \mathbf{0}\right), \\
\mathbf{0}= & \frac{1}{m^{2}} \Omega^{2} \mathbf{k}_{2} \mathbf{c}^{(m) *}+\mathbf{F}_{2}\left(\mathbf{a}_{0}^{(m) *}, \mathbf{b}^{(m) *}, \mathbf{c}^{(m) *}, \mathbf{0}, \mathbf{0}, \mathbf{0} ;\right. \\
& \left.\mathbf{a}_{0}^{\tau(m) *}, \mathbf{b}^{\tau(m) *}, \mathbf{c}^{\tau(m) *}, \mathbf{0}, \mathbf{0}, \mathbf{0}\right) .
\end{aligned}
$$

with

$\mathbf{a}_{0}^{(m) *}=\mathbf{a}_{0}^{\tau(m) *}, \mathbf{b}^{(m) *}=\mathbf{b}^{\tau(m) *}, \mathbf{c}^{(m) *}=\mathbf{c}^{\tau(m) *}$.

Thus, the existing solutions of the forgoing equation are the existence conditions of the periodic solutions for timedelayed, nonlinear dynamical systems. If the foregoing equation gives equilibrium $\mathbf{y}^{(m) *}=\mathbf{y}^{\tau(m) *}$, in vicinity of $\mathbf{y}^{(m) *}=$ $\mathbf{y}^{\tau(m) *}$, with $\mathbf{y}^{(m)}=\mathbf{y}^{(m) *}+\Delta \mathbf{y}^{(m)}$ and $\mathbf{y}^{\tau(m)}=\mathbf{y}^{\tau(m) *}+$ 
$\Delta \mathbf{y}^{\tau(m)}$, the linearized equation of $\dot{\mathbf{y}}^{(m)}=\mathbf{f}^{(m)}\left(\mathbf{y}^{(m)}, \mathbf{y}^{\tau(m)}\right)$ is

$$
\begin{aligned}
& \Delta \dot{\mathbf{y}}^{(m)}=D_{\mathbf{y}^{(m)}} \mathbf{f}^{(m)}\left(\mathbf{y}^{(m) *}, \mathbf{y}^{\tau(m) *}\right) \\
& \Delta \mathbf{y}^{(m)}+D_{\mathbf{y}^{\tau(m)}} \mathbf{f}^{(m)}\left(\mathbf{y}^{(m) *}, \mathbf{y}^{\tau(m) *}\right) \Delta \mathbf{y}^{\tau(m)}
\end{aligned}
$$

and the eigenvalue analysis of equilibrium $\mathbf{y}^{(m) *}=\mathbf{y}^{\tau(m) *}$ is given by

$$
\begin{aligned}
& \mid D_{\mathbf{y}^{(m)}} \mathbf{f}^{(m)}\left(\mathbf{y}^{(m) *}, \mathbf{y}^{\tau(m) *}\right)-\lambda \mathbf{I}_{2 n(2 N+1) \times 2 n(2 N+1)} \\
& \quad+D_{\mathbf{y}^{\tau(m)}} \mathbf{f}^{(m)}\left(\mathbf{y}^{(m) *}, \mathbf{y}^{\tau(m) *}\right) e^{-\lambda \tau} \mid=0
\end{aligned}
$$

where

$$
\begin{aligned}
& D_{\mathbf{y}^{(m)}} \mathbf{f}^{(m)}\left(\mathbf{y}^{(m) *}, \mathbf{y}^{\tau(m) *}\right) \\
& \quad=\partial \mathbf{f}^{(m)}\left(\mathbf{y}^{(m)}, \mathbf{y}^{\tau(m)}\right) /\left.\partial \mathbf{y}^{(m)}\right|_{\left(\mathbf{y}^{(m) *}, \mathbf{y}^{\tau(m) *}\right)}, \\
& D_{\mathbf{y}^{\tau(m)}} \mathbf{f}^{(m)}\left(\mathbf{y}^{(m) *}, \mathbf{y}^{\tau(m) *}\right) \\
& =\partial \mathbf{f}^{(m)}\left(\mathbf{y}^{(m)}, \mathbf{y}^{\tau(m)}\right) /\left.\partial \mathbf{y}^{\tau(m)}\right|_{\left(\mathbf{y}^{(m) *}, \mathbf{y}^{\tau(m) *}\right)}
\end{aligned}
$$

The periodic solution stability and bifurcation can be classified by the eigenvalues of Eq. (181) at equilibrium $\mathbf{y}^{(m) *}=$ $\mathbf{y}^{\tau(m) *}$ with

$\left(n_{1}, n_{2}, n_{3} \mid n_{4}, n_{5}, n_{6}\right)$.

From the stability and bifurcation theory of dynamical systems at equilibriums, the stability and bifurcation of periodic solutions can be classified as stated in the theorem. This theorem is proved.

If the Hopf bifurcation of period- $m$ motion occurs, the period-doubling solution of period- $m$ motion can be expressed by

$$
\begin{aligned}
\mathbf{x}^{(2 m) *}(t)= & \mathbf{a}_{0}^{(2 m)}(t)+\sum_{k=1}^{N} \mathbf{b}_{k / 2 m}(t) \cos \left(\frac{k}{2 m} \Omega t\right) \\
& +\mathbf{c}_{k / 2 m}(t) \sin \left(\frac{k}{2 m} \Omega t\right) \\
\mathbf{x}^{(2 m) \tau *}(t)= & \mathbf{a}_{0}^{\tau(2 m)}(t)+\sum_{k=1}^{N} \mathbf{b}_{k / 2 m}^{\tau}(t) \cos \left[\frac{k}{2 m} \Omega(t-\tau)\right] \\
& +\mathbf{c}_{k / 2 m}^{\tau}(t) \sin \left[\frac{k}{2 m} \Omega(t-\tau)\right]
\end{aligned}
$$

where for $k=1,2, \ldots, N$

$$
\begin{gathered}
\ddot{\mathbf{a}}_{0}^{(2 m)}=\mathbf{F}_{0}^{(2 m)}\left(\mathbf{z}^{(2 m)}, \mathbf{z}_{1}^{(2 m)} ; \mathbf{z}^{\tau(2 m)}, \mathbf{z}_{1}^{\tau(2 m)}\right), \\
\ddot{\mathbf{b}}^{(2 m)}+2 \frac{\Omega}{(2 m)} \mathbf{k}_{1} \dot{\mathbf{c}}^{(2 m)}-\frac{\Omega^{2}}{(2 m)^{2}} \mathbf{k}_{2} \mathbf{b}^{(2 m)} \\
=\mathbf{F}_{1 k}^{(m)}\left(\mathbf{z}^{(2 m)}, \mathbf{z}_{1}^{(2 m)} ; \mathbf{z}^{\tau(2 m)}, \mathbf{z}_{1}^{\tau(2 m)}\right), \\
\ddot{\mathbf{c}}^{(2 m)}-2 \frac{\Omega}{(2 m)} \mathbf{k}_{1} \dot{\mathbf{b}}^{(2 m)}-\frac{\Omega^{2}}{(2 m)^{2}} \mathbf{k}_{2} \mathbf{c}^{(2 m)} \\
=\mathbf{F}_{2 k}^{(m)}\left(\mathbf{z}^{(2 m)}, \mathbf{z}_{1}^{(2 m)} ; \mathbf{z}^{\tau(2 m)}, \mathbf{z}_{1}^{\tau(2 m)}\right)
\end{gathered}
$$

where for $N=1,2, \ldots, \infty$.

$$
\begin{aligned}
& \mathbf{k}_{1}=\operatorname{diag}\left(\mathbf{I}_{n \times n}, 2 \mathbf{I}_{n \times n}, \ldots, N \mathbf{I}_{n \times n}\right), \\
& \mathbf{k}_{2}=\operatorname{diag}\left(\mathbf{I}_{n \times n}, 2^{2} \mathbf{I}_{n \times n}, \ldots, N^{2} \mathbf{I}_{n \times n}\right) ; \\
& \mathbf{b}^{(2 m)}=\left(\mathbf{b}_{1 / 2 m}, \mathbf{b}_{2 / 2 m}, \ldots, \mathbf{b}_{N / 2 m}\right)^{\mathrm{T}}, \\
& \mathbf{c}^{(2 m)}=\left(\mathbf{c}_{1 / 2 m}, \mathbf{c}_{2 / 2 m}, \ldots, \mathbf{c}_{N / 2 m}\right)^{\mathrm{T}} ; \\
& \mathbf{b}^{\tau(2 m)}=\left(\mathbf{b}_{1 / 2 m}^{\tau}, \mathbf{b}_{2 / 2 m}^{\tau}, \ldots, \mathbf{b}_{N / 2 m}^{\tau}\right)^{\mathrm{T}}, \\
& \mathbf{c}^{\tau(2 m)}=\left(\mathbf{c}_{1 / 2 m}^{\tau}, \mathbf{c}_{2 / 2 m}^{\tau}, \ldots, \mathbf{c}_{N / 2 m}^{\tau}\right)^{\mathrm{T}} ; \\
& \mathbf{F}_{1}^{(2 m)}=\left(\mathbf{F}_{11}^{(2 m)}, \mathbf{F}_{12}^{(2 m)}, \ldots, \mathbf{F}_{1 N}^{(2 m)}\right)^{\mathrm{T}}, \\
& \mathbf{F}_{2}^{(2 m)}=\left(\mathbf{F}_{21}^{(2 m)}, \mathbf{F}_{22}^{(2 m)}, \ldots, \mathbf{F}_{2 N}^{(2 m)}\right)^{\mathrm{T}} ; \\
& \mathbf{z}^{(2 m)}=\left(\mathbf{a}_{0}^{(2 m)}, \mathbf{b}^{(2 m)}, \mathbf{c}^{(2 m)}\right)^{\mathrm{T}}, \dot{\mathbf{z}}^{(2 m)}=\mathbf{z}_{1}^{(2 m) ;} \\
& \mathbf{z}^{\tau(2 m)}=\left(\mathbf{a}_{0}^{\tau(2 m)}, \mathbf{b}^{\tau(2 m)}, \mathbf{c}^{\tau(2 m)}\right)^{\mathrm{T}}, \dot{\mathbf{z}}^{\tau(2 m)}=\mathbf{z}_{1}^{\tau(2 m)}
\end{aligned}
$$

and for $k=1,2, \ldots, N$

$$
\begin{aligned}
& \mathbf{F}_{0}^{(2 m)}\left(\mathbf{z}^{(2 m)}, \mathbf{z}_{1}^{(2 m)} ; \mathbf{z}^{\tau(2 m)}, \mathbf{z}_{1}^{\tau(2 m)}\right) \\
& =\frac{1}{2 m T} \int_{0}^{2 m T} \mathbf{f}\left(\mathbf{x}^{(2 m) *}, \dot{\mathbf{x}}^{(2 m) *} ; \mathbf{x}^{\tau(2 m) *}, \dot{\mathbf{x}}^{\tau(2 m) *}, \mathbf{p}\right) d t \\
& \mathbf{F}_{1 k}^{(2 m)}\left(\mathbf{z}^{(2 m)}, \mathbf{z}_{1}^{(2 m)} ; \mathbf{z}^{\tau(2 m)}, \mathbf{z}_{1}^{\tau(2 m)}\right) \\
& =\frac{1}{m T} \int_{0}^{2 m T} \mathbf{f}\left(\mathbf{x}^{(2 m) *}, \dot{\mathbf{x}}^{(2 m) *} ; \mathbf{x}^{\tau(2 m) *}, \dot{\mathbf{x}}^{\tau(2 m) *}, \mathbf{p}\right) \\
& \quad \times \cos \left(\frac{k}{2 m} \Omega t\right) d t, \\
& \mathbf{F}_{2 k}^{(2 m)}\left(\mathbf{z}^{(2 m)}, \mathbf{z}_{1}^{(2 m)} ; \mathbf{z}^{\tau(2 m)}, \mathbf{z}_{1}^{\tau(2 m)}\right) \\
& =\frac{1}{m T} \int_{0}^{2 m T} \mathbf{f}\left(\mathbf{x}^{(2 m) *}, \dot{\mathbf{x}}^{(2 m) *} ; \mathbf{x}^{\tau(2 m) *}, \dot{\mathbf{x}}^{\tau(2 m) *}, \mathbf{p}\right) \\
& \quad \times \sin \left(\frac{k}{2 m} \Omega t\right) d t
\end{aligned}
$$

If the Hopf bifurcation of period- $2 m$ motion occurs again and again, the analytical expression for period- $2^{l} m$ motion can be expressed by

$$
\begin{aligned}
\mathbf{x}^{\left(2^{l} m\right) *}(t)= & \mathbf{a}_{0}^{\left(2^{l} m\right)}(t)+\sum_{k=1}^{N} \mathbf{b}_{k / 2^{l} m}(t) \cos \left(\frac{k}{2^{l} m} \Omega t\right) \\
& +\mathbf{c}_{k / 2^{l} m}(t) \sin \left(\frac{k}{2^{l} m} \Omega t\right), \\
\mathbf{x}^{\left(2^{l} m\right) \tau *}(t)= & \mathbf{a}_{0}^{\tau\left(2^{l} m\right)}(t)+\sum_{k=1}^{N} \mathbf{b}_{k / 2^{2} m}^{\tau}(t) \cos \left[\frac{k}{2^{l} m} \Omega(t-\tau)\right] \\
& +\mathbf{c}_{k / 2^{l} m}^{\tau}(t) \sin \left[\frac{k}{2^{l} m} \Omega(t-\tau)\right],
\end{aligned}
$$

where for $k=1,2, \ldots, N$

$\ddot{\mathbf{a}}_{0}^{\left(2^{l} m\right)}=\mathbf{F}_{0}^{\left(2^{l} m\right)}\left(\mathbf{z}^{\left(2^{l} m\right)}, \mathbf{z}_{1}^{\left(2^{l} m\right)} ; \mathbf{z}^{\tau\left(2^{l} m\right)}, \mathbf{z}_{1}^{\tau\left(2^{l} m\right)}\right)$, 


$$
\begin{array}{r}
\ddot{\mathbf{b}}^{\left(2^{l} m\right)}+2 \frac{\Omega}{2^{l} m} \mathbf{k}_{1} \dot{\mathbf{c}}^{\left(2^{l} m\right)}-\frac{\Omega^{2}}{\left(2^{l} m\right)^{2}} \mathbf{k}_{2} \mathbf{b}^{\left(2^{l} m\right)} \\
=\mathbf{F}_{1 k}^{\left(2^{l} m\right)}\left(\mathbf{z}^{\left(2^{l} m\right)}, \mathbf{z}_{1}^{\left(2^{l} m\right)} ; \mathbf{z}^{\tau\left(2^{l} m\right)}, \mathbf{z}_{1}^{\tau\left(2^{l} m\right)}\right), \\
\ddot{\mathbf{c}}^{\left(2^{l} m\right)}-2 \frac{\Omega}{2^{l} m} \mathbf{k}_{1} \dot{\mathbf{b}}^{\left(2^{l} m\right)}-\frac{\Omega^{2}}{\left(2^{l} m\right)^{2}} \mathbf{k}_{2} \mathbf{c}^{\left(2^{l} m\right)} \\
\quad=\mathbf{F}_{2 k}^{\left(2^{l} m\right)}\left(\mathbf{z}^{\left(2^{l} m\right)}, \mathbf{z}_{1}^{\left(2^{l} m\right)} ; \mathbf{z}^{\tau\left(2^{l} m\right)}, \mathbf{z}_{1}^{\tau\left(2^{l} m\right)}\right) ;
\end{array}
$$

and for $N=1,2, \ldots, \infty$

$$
\begin{aligned}
& \mathbf{k}_{1}=\operatorname{diag}\left(\mathbf{I}_{n \times n}, 2 \mathbf{I}_{n \times n}, \ldots, N \mathbf{I}_{n \times n}\right), \\
& \mathbf{k}_{2}=\operatorname{diag}\left(\mathbf{I}_{n \times n}, 2^{2} \mathbf{I}_{n \times n}, \ldots, N^{2} \mathbf{I}_{n \times n}\right), \\
& \mathbf{b}^{\left(2^{l} m\right)}=\left(\mathbf{b}_{1 / 2^{l} m}, \mathbf{b}_{2 / 2^{l} m}, \ldots, \mathbf{b}_{N / 2^{l} m}\right)^{\mathrm{T}}, \\
& \mathbf{c}^{\left(2^{l} m\right)}=\left(\mathbf{c}_{1 / 2^{l} m}, \mathbf{c}_{2 / 2^{l} m}, \ldots, \mathbf{c}_{N / 2^{l} m}\right)^{\mathrm{T}} ; \\
& \mathbf{b}^{\tau\left(2^{l} m\right)}=\left(\mathbf{b}_{1 / 2^{l} m}^{\tau}, \mathbf{b}_{2 / 2^{l} m}^{\tau}, \ldots, \mathbf{b}_{N / 2^{l} m}^{\tau}\right)^{\mathrm{T}}, \\
& \mathbf{c}^{\tau\left(2^{l} m\right)}=\left(\mathbf{c}_{1 / 2^{l} m}^{\tau}, \mathbf{c}_{2 / 2^{l} m}^{\tau}, \ldots, \mathbf{c}_{N / 2^{l} m}^{\tau}\right)^{\mathrm{T}} ; \\
& \mathbf{F}_{1}^{\left(2^{l} m\right)}=\left(\mathbf{F}_{11}^{\left(2^{l} m\right)}, \mathbf{F}_{12}^{\left(2^{l} m\right)}, \ldots, \mathbf{F}_{1 N}^{\left(2^{l} m\right)}\right)^{\mathrm{T}}, \\
& \mathbf{F}_{2}^{\left(2^{l} m\right)}=\left(\mathbf{F}_{21}^{\left(2^{l} m\right)}, \mathbf{F}_{22}^{\left(2^{l} m\right)}, \ldots, \mathbf{F}_{2 N}^{\left(2^{l} m\right)}\right)^{\mathrm{T}} ; \\
& \mathbf{z}^{\left(2^{l} m\right)}=\left(\mathbf{a}_{0}^{\left(2^{l} m\right)}, \mathbf{b}^{\left(2^{l} m\right)}, \mathbf{c}^{\left(2^{l} m\right)}\right)^{\mathrm{T}}, \dot{\mathbf{z}}^{\left(2^{l} m\right)}=\mathbf{z}_{1}^{\left(2^{l} m\right)} ; \\
& \mathbf{z}^{\tau\left(2^{l} m\right)}=\left(\mathbf{a}_{0}^{\tau\left(2^{l} m\right)}, \mathbf{b}^{\tau\left(2^{l} m\right)}, \mathbf{c}^{\tau\left(2^{l} m\right)}\right)^{\mathrm{T}}, \dot{\mathbf{z}}^{\tau\left(2^{l} m\right)}=\mathbf{z}_{1}^{\tau\left(2^{l} m\right)}
\end{aligned}
$$

$$
\begin{aligned}
& \mathbf{F}_{0}^{\left(2^{l} m\right)}\left(\mathbf{z}^{\left(2^{l} m\right)}, \mathbf{z}_{1}^{\left(2^{l} m\right)} ; \mathbf{z}^{\tau\left(2^{l} m\right)}, \mathbf{z}_{1}^{\tau\left(2^{l} m\right)}\right) \\
& =\frac{1}{2^{l} m T} \int_{0}^{2^{l} m T} \mathbf{f}\left(\mathbf{x}^{\left(2^{l} m\right) *}, \dot{\mathbf{x}}^{\left(2^{l} m\right) *} ; \mathbf{x}^{\tau\left(2^{l} m\right) *}, \dot{\mathbf{x}}^{\tau\left(2^{l} m\right) *}, \mathbf{p}\right) d t ; \\
& \mathbf{F}_{1 k}^{\left(2^{l} m\right)}\left(\mathbf{z}^{\left(2^{l} m\right)}, \mathbf{z}_{1}^{\left(2^{l} m\right)} ; \mathbf{z}^{\tau\left(2^{l} m\right)}, \mathbf{z}_{1}^{\tau\left(2^{l} m\right)}\right) \\
& =\frac{1}{2^{l-1} m T} \int_{0}^{2^{l} m T} \mathbf{f}\left(\mathbf{x}^{\left(2^{l} m\right) *}, \dot{\mathbf{x}}^{\left(2^{l} m\right) *} ; \mathbf{x}^{\tau\left(2^{l} m\right) *}, \dot{\mathbf{x}}^{\tau\left(2^{l} m\right) *}, \mathbf{p}\right) \\
& \times \cos \left(\frac{k}{2^{l} m} \Omega t\right) d t, \\
& \mathbf{F}_{2 k}^{\left(2^{l} m\right)}\left(\mathbf{z}^{\left(2^{l} m\right)}, \mathbf{z}_{1}^{\left(2^{l} m\right)} ; \mathbf{z}^{\tau\left(2^{l} m\right)}, \mathbf{z}_{1}^{\tau\left(2^{l} m\right)}\right) \\
& =\frac{1}{2^{l-1} m T} \int_{0}^{2^{l} m T} \mathbf{f}\left(\mathbf{x}^{\left(2^{l} m\right) *}, \dot{\mathbf{x}}^{\left(2^{l} m\right) *} ; \mathbf{x}^{\tau\left(2^{l} m\right) *}, \dot{\mathbf{x}}^{\tau\left(2^{l} m\right) *}, \mathbf{p}\right) \\
& \times \sin \left(\frac{k}{2^{l} m} \Omega t\right) d t \text {. }
\end{aligned}
$$

The solution of period- $2^{l} m$ motion can be determined by equilibriums of coefficient dynamical system in Eq. (190), and the corresponding stability and bifurcation can be done. As $l \rightarrow \infty$, the stable and unstable chaos with $\left(n_{1}, n_{2}, n_{3} \mid n_{4}, n_{5}, n_{6}\right)$ in Eq. (184) can be obtained where

$$
\sum_{i=1}^{3} n_{i}+2 \sum_{i=4}^{6} n_{i}=2 n(1+2 N)
$$

With increasing $l$, the Fourier truncated number $N \gg 2^{l} m$ will dramatically increase. If period- 1 motion possess at least $N_{1}$ harmonic vector terms, then the total harmonic vector terms for period- $2^{l} m$ motion should be $N \geq 2^{l} m N_{1}$. The chaotic motion classifications with specific cases for the timedelayed systems are given as follows.

(i) For the chaotic motion of $\left(n_{1}, 0,0 \mid 0,0,0\right)$ with $n_{1}=$ $2 n(1+2 N)$, the chaotic motion is called the hyperbolic stable chaos.

(ii) For the chaotic motion of $\left(0,0,0 \mid n_{4}, 0,0\right)$ with $n_{4}=$ $n(1+2 N)$, the chaotic motion is called the spiral stable chaos.

(iii) For the chaotic motion of $\left(n_{1}, 0,0 \mid n_{4}, 0,0\right)$ with $n_{1}+$ $2 n_{4}=2 n(1+2 N)$, the chaotic motion is called the hyperbolic-spiral stable chaos.

(iv) For the chaotic motion of $\left(0, n_{2}, 0 \mid 0,0,0\right)$ with $n_{2}=$ $2 n(1+2 N)$, the chaotic motion is called the hyperbolic unstable chaos.

(v) For the chaotic motion of $\left(0,0,0 \mid 0, n_{5}, 0\right)$ with $n_{5}=$ $n(1+2 N)$, the chaotic motion is called the spiral unstable chaos.

(vi) For the chaotic motion of $\left(0, n_{2}, 0 \mid 0, n_{5}, 0\right)$ with $n_{2}+$ $2 n_{5}=2 n(1+2 N)$, the chaotic motion is called the hyperbolic-spiral unstable chaos.

(vii) For the chaotic motion of $\left(n_{1}, n_{2}, 0 \mid 0,0,0\right)$ with $n_{1}+$ $n_{2}=2 n(1+2 N)$, the chaotic motion is called the saddle unstable chaos.

(viii) For the chaotic motion of $\left(n_{1}, n_{2}, 0 \mid n_{4}, n_{5}, 0\right)$ with $n_{1}+n_{2}+2 n_{4}+2 n_{5}=2 n(1+2 N)$, the chaotic motion is called the spiral saddle unstable chaos.

Because $m$ is an arbitrary positive integer number, it includes $\left(2^{l} m_{1}\right)$ for period- $2^{l} m_{1}$ motion. Thus, the expression for period- $m$ motion can be used for any periodic motions. The expression in Eq. (172) can be used to express the solution for chaotic motion as $m \rightarrow \infty$. The chaotic solutions in the time-delayed, nonlinear vibration systems can be classified as discussed before for period- $2^{l} m_{1}$ motion.

\subsection{Periodically forced, time-delayed, vibration systems}

Periodic flows in periodically forced, time-delayed, vibration systems will be discussed herein. If such forced, timedelayed, vibration systems possess periodic motions with period $T=2 \pi / \Omega$, then such periodic motions can be expressed by a Fourier series, discussed as follows.

Theorem 15 Consider a periodically forced, time-delayed, nonlinear vibration system as

$\ddot{\mathbf{x}}=\mathbf{F}\left(\mathbf{x}, \dot{\mathbf{x}} ; \mathbf{x}^{\tau}, \dot{\mathbf{x}}^{\tau}, t, \mathbf{p}\right) \in \mathbf{R}^{n}$ 
where $\mathbf{F}\left(\mathbf{x}, \dot{\mathbf{x}} ; \mathbf{x}^{\tau}, \dot{\mathbf{x}}^{\tau}, t, \mathbf{p}\right)$ is a $C^{r}$-continuous nonlinear function vector ( $r \geq 1$ ) with forcing period $T=2 \pi / \Omega$. If there is a periodic motion $\mathbf{x}^{*}(t)$ with finite norm $\left\|\mathbf{x}^{*}\right\|$, an analytical expression for the periodic flow of Eq. (194) is in the form of

$$
\begin{aligned}
\mathbf{x}^{*}= & \mathbf{a}_{0}(t)+\sum_{k=1}^{N} \mathbf{b}_{k}(t) \cos (k \Omega t)+\mathbf{c}_{k}(t) \sin (k \Omega t), \\
\mathbf{x}^{\tau *}= & \mathbf{a}_{0}^{\tau}(t)+\sum_{k=1}^{N} \mathbf{b}_{k}^{\tau}(t) \cos [k \Omega(t-\tau)] \\
& +\mathbf{c}_{k}^{\tau}(t) \sin [k \Omega(t-\tau)]
\end{aligned}
$$

with

$$
\begin{aligned}
\mathbf{a}_{0} & =\left(a_{01}, a_{02}, \ldots, a_{0 n}\right)^{\mathrm{T}}, \\
\mathbf{b}_{k} & =\left(b_{k 1}, b_{k 2}, \ldots, b_{k n}\right)^{\mathrm{T}}, \\
\mathbf{c}_{k} & =\left(c_{k 1}, c_{k 2}, \ldots, c_{k n}\right)^{\mathrm{T}} ; \\
\mathbf{a}_{0}^{\tau} & =\left(a_{01}^{\tau}, a_{02}^{\tau}, \ldots, a_{0 n}^{\tau}\right)^{\mathrm{T}}, \\
\mathbf{b}_{k}^{\tau} & =\left(b_{k 1}^{\tau}, b_{k 2}^{\tau}, \ldots, b_{k n}^{\tau}\right)^{\mathrm{T}}, \\
\mathbf{c}_{k}^{\tau} & =\left(c_{k 1}^{\tau}, c_{k 2}^{\tau}, \ldots, c_{k n}^{\tau}\right)^{\mathrm{T}} ;
\end{aligned}
$$

and the corresponding coefficients slowly varying with time are determined by

$$
\begin{aligned}
& \ddot{\mathbf{a}}_{0}=\mathbf{F}_{0}\left(\mathbf{z}, \mathbf{z}_{1} ; \mathbf{z}^{\tau}, \mathbf{z}_{1}^{\tau}\right), \\
& \ddot{\mathbf{b}}=-2 \Omega \mathbf{k}_{1} \dot{\mathbf{c}}+\Omega^{2} \mathbf{k}_{2} \mathbf{b}+\mathbf{F}_{1}\left(\mathbf{z}, \mathbf{z}_{1} ; \mathbf{z}^{\tau}, \mathbf{z}_{1}^{\tau}\right), \\
& \ddot{\mathbf{c}}=2 \Omega \mathbf{k}_{1} \dot{\mathbf{b}}+\Omega^{2} \mathbf{k}_{2} \mathbf{c}+\mathbf{F}_{2}\left(\mathbf{z}, \mathbf{z}_{1} ; \mathbf{z}^{\tau}, \mathbf{z}_{1}^{\tau}\right) \\
& \text { where for } N=1,2, \ldots, \infty .
\end{aligned}
$$

$$
\begin{aligned}
& \mathbf{k}_{1}=\operatorname{diag}\left(\mathbf{I}_{n \times n}, 2 \mathbf{I}_{n \times n}, \ldots, N \mathbf{I}_{n \times n}\right), \\
& \mathbf{k}_{2}=\operatorname{diag}\left(\mathbf{I}_{n \times n}, 2^{2} \mathbf{I}_{n \times n}, \ldots, N^{2} \mathbf{I}_{n \times n}\right) \\
& \mathbf{b}=\left(\mathbf{b}_{1}, \mathbf{b}_{2}, \ldots, \mathbf{b}_{N}\right)^{\mathrm{T}}, \\
& \mathbf{c}=\left(\mathbf{c}_{1}, \mathbf{c}_{2}, \ldots, \mathbf{c}_{N}\right)^{\mathrm{T}} ; \\
& \mathbf{b}^{\tau}=\left(\mathbf{b}_{1}^{\tau}, \mathbf{b}_{2}^{\tau}, \ldots, \mathbf{b}_{N}^{\tau}\right)^{\mathrm{T}}, \\
& \mathbf{c}^{\tau}=\left(\mathbf{c}_{1}^{\tau}, \mathbf{c}_{2}^{\tau}, \ldots, \mathbf{c}_{N}^{\tau}\right)^{\mathrm{T}} ; \\
& \mathbf{F}_{1}=\left(\mathbf{F}_{11}, \mathbf{F}_{12}, \ldots, \mathbf{F}_{1 N}\right)^{\mathrm{T}}, \\
& \mathbf{F}_{2}=\left(\mathbf{F}_{21}, \mathbf{F}_{22}, \ldots, \mathbf{F}_{2 N}\right)^{\mathrm{T}} ; \\
& \mathbf{z}=\left(\mathbf{a}_{0}, \mathbf{b}, \mathbf{c}\right)^{\mathrm{T}}, \dot{\mathbf{z}}=\mathbf{z}_{1} ; \\
& \mathbf{z}^{\tau}=\left(\mathbf{a}_{0}^{\tau}, \mathbf{b}^{\tau}, \mathbf{c}^{\tau}\right)^{\mathrm{T}}, \dot{\mathbf{z}}^{\tau}=\mathbf{z}_{1}^{\tau} \\
& \text { and for } k=1,2, \ldots, N
\end{aligned}
$$

$\mathbf{F}_{0}\left(\mathbf{z}, \mathbf{z}_{1} ; \mathbf{z}^{\tau}, \mathbf{z}_{1}^{\tau}\right)=\frac{1}{T} \int_{0}^{T} \mathbf{F}\left(\mathbf{x}^{*}, \dot{\mathbf{x}}^{*} ; \mathbf{x}^{\tau *}, \dot{\mathbf{x}}^{\tau *}, t, \mathbf{p}\right) d t ;$

$\mathbf{F}_{1 k}\left(\mathbf{z}, \mathbf{z}_{1} ; \mathbf{z}^{\tau}, \mathbf{z}_{1}^{\tau}\right)$

$$
=\frac{2}{T} \int_{0}^{T} \mathbf{F}\left(\mathbf{x}^{*}, \dot{\mathbf{x}}^{*} ; \mathbf{x}^{\tau *}, \dot{\mathbf{x}}^{\tau *}, t, \mathbf{p}\right) \cos (k \Omega t) d t,
$$

$\mathbf{F}_{2 k}\left(\mathbf{z}, \mathbf{z}_{1} ; \mathbf{z}^{\tau}, \mathbf{z}_{1}^{\tau}\right)$

$$
=\frac{2}{T} \int_{0}^{T} \mathbf{F}\left(\mathbf{x}^{*}, \dot{\mathbf{x}}^{*} ; \mathbf{x}^{\tau *}, \dot{\mathbf{x}}^{\tau *}, t, \mathbf{p}\right) \sin (k \Omega t) d t .
$$

The state-space form of Eq. (197) is

$\dot{\mathbf{z}}=\mathbf{z}_{1} \quad$ and $\quad \dot{\mathbf{z}}_{1}=\mathbf{g}\left(\mathbf{z}, \mathbf{z}_{1} ; \mathbf{z}^{\tau}, \mathbf{z}_{1}^{\tau}\right)$

where

$$
\begin{aligned}
\mathbf{g}= & \left(\mathbf{F}_{0},-2 \Omega \mathbf{k}_{1} \dot{\mathbf{c}}+\Omega^{2} \mathbf{k}_{2} \mathbf{b}+\mathbf{F}_{1},\right. \\
& \left.2 \Omega \mathbf{k}_{1} \dot{\mathbf{b}}+\Omega^{2} \mathbf{k}_{2} \mathbf{c}+\mathbf{F}_{2}\right)^{\mathrm{T}} .
\end{aligned}
$$

An equivalent system of Eq. (200) is

$\dot{\mathbf{y}}=\mathbf{f}\left(\mathbf{y}, \mathbf{y}^{\tau}\right)$

where

$\mathbf{y}=\left(\mathbf{z}, \mathbf{z}_{1}\right)^{\mathrm{T}}, \mathbf{y}^{\tau}=\left(\mathbf{z}^{\tau}, \mathbf{z}_{1}^{\tau}\right)^{\mathrm{T}}$ and $\mathbf{f}=\left(\mathbf{z}_{1}, \mathbf{g}\right)^{\mathrm{T}}$

If equilibrium $\mathbf{y}^{*}=\mathbf{y}^{\tau *}$ of Eq. (202) (i.e., $\mathbf{f}\left(\mathbf{y}^{*}, \mathbf{y}^{\tau *}\right)=\mathbf{0}$ ) exists, then the approximate analytical solution of periodic motion exists in Eq. (195). In vicinity of equilibrium $\mathbf{y}^{*}=\mathbf{y}^{\tau *}$, with $\mathbf{y}=\mathbf{y}^{*}+\Delta \mathbf{y}$ and $\mathbf{y}^{\tau}=\mathbf{y}^{\tau *}+\Delta \mathbf{y}^{\tau}$, the linearized equation of Eq. (202) is

$\Delta \dot{\mathbf{y}}=D_{\mathbf{y}} \mathbf{f}\left(\mathbf{y}^{*}, \mathbf{y}^{\tau *}\right) \Delta \mathbf{y}+D_{\mathbf{y}^{\tau}} \mathbf{f}\left(\mathbf{y}^{*}, \mathbf{y}^{\tau *}\right) \Delta \mathbf{y}^{\tau}$

and the eigenvalue analysis of equilibrium $\mathbf{y}^{*}$ is given by

$$
\begin{aligned}
& \mid D_{\mathbf{y}} \mathbf{f}\left(\mathbf{y}^{*}, \mathbf{y}^{\tau *}\right)-\lambda \mathbf{I}_{2 n(2 N+1) \times 2 n(2 N+1)} \\
& +D_{\mathbf{y}^{\tau}} \mathbf{f}\left(\mathbf{y}^{*}, \mathbf{y}^{\tau *}\right) e^{-\lambda \tau} \mid=0
\end{aligned}
$$

where

$$
\begin{aligned}
& D_{\mathbf{y}} \mathbf{f}\left(\mathbf{y}^{*}, \mathbf{y}^{\tau *}\right)=\left.\frac{\partial \mathbf{f}\left(\mathbf{y}, \mathbf{y}^{\tau}\right)}{\partial \mathbf{y}}\right|_{\left(\mathbf{y}^{*}, \mathbf{y}^{\tau *}\right)}, \\
& D_{\mathbf{y}^{\tau}} \mathbf{f}\left(\mathbf{y}^{*}, \mathbf{y}^{\tau *}\right)=\left.\frac{\partial \mathbf{f}\left(\mathbf{y}, \mathbf{y}^{\tau}\right)}{\partial \mathbf{y}^{\tau}}\right|_{\left(\mathbf{y}^{*}, \mathbf{y}^{\tau *}\right)} .
\end{aligned}
$$

Thus, the eigenvalues of Eq. (204) are classified by

$\left(n_{1}, n_{2}, n_{3} \mid n_{4}, n_{5}, n_{6}\right)$

(i) If all eigenvalues of the equilibrium possess negative real parts, the approximate periodic solution is stable.

(ii) If at least one of eigenvalues of the equilibrium possesses positive real part, the approximate periodic solution is unstable.

(iii) The boundaries between stable and unstable equilibriums with higher order singularity give bifurcation and stability conditions with higher order singularity.

Proof The proof of this theorem is similar to Theorem 13.

Similarly, the periodic- $m$ flow in dynamical system will be discussed. 
Theorem 16 Consider a periodically forced, time-dleyaed, nonlinear vibration system in Eq. (194) with a forcing period $T=2 \pi / \Omega$. If there is a period-m motion $\mathbf{x}^{(m) *}(t)$ with finite norm $\left\|\mathbf{x}^{(m) *}\right\|$, an analytical expression for the period$m$ motion of Eq. (194) is

$$
\begin{aligned}
\mathbf{x}^{(m) *}(t)= & \mathbf{a}_{0}^{(m)}(t)+\sum_{k=1}^{N} \mathbf{b}_{k / m}(t) \cos \left(\frac{k}{m} \Omega t\right) \\
& +\mathbf{c}_{k / m}(t) \sin \left(\frac{k}{m} \Omega t\right) ; \\
\mathbf{x}^{\tau(m) *}(t)= & \mathbf{a}_{0}^{\tau(m)}(t)+\sum_{k=1}^{N} \mathbf{b}_{k / m}^{\tau}(t) \cos \left[\frac{k}{m} \Omega(t-\tau)\right] \\
& +\mathbf{c}_{k / m}^{\tau}(t) \sin \left[\frac{k}{m} \Omega(t-\tau)\right]
\end{aligned}
$$

with for $k=1,2, \ldots, N$

$$
\begin{aligned}
& \mathbf{a}_{0}^{(m)}=\left(a_{01}^{(m)}, a_{02}^{(m)}, \ldots, a_{0 n}^{(m)}\right)^{\mathrm{T}}, \\
& \mathbf{b}_{k / m}=\left(b_{k / m 1}, b_{k / m 2}, \ldots, b_{k / m n}\right)^{\mathrm{T}}, \\
& \mathbf{c}_{k / m}=\left(c_{k / m 1}, c_{k / m 2}, \ldots, c_{k / m n}\right)^{\mathrm{T}} ; \\
& \mathbf{a}_{0}^{\tau(m)}=\left(a_{01}^{\tau(m)}, a_{02}^{\tau(m)}, \ldots, a_{0 n}^{\tau(m)}\right)^{\mathrm{T}}, \\
& \mathbf{b}_{k / m}^{\tau}=\left(b_{k / m 1}^{\tau}, b_{k / m 2}^{\tau}, \ldots, b_{k / m n}^{\tau}\right)^{\mathrm{T}}, \\
& \mathbf{c}_{k / m}^{\tau}=\left(c_{k / m 1}^{\tau}, c_{k / m 2}^{\tau}, \ldots, c_{k / m n}^{\tau}\right)^{\mathrm{T}}
\end{aligned}
$$

and the corresponding coefficients slowly varying with time are determined by

$$
\begin{aligned}
\ddot{\mathbf{a}}_{0}^{(m)}= & \mathbf{F}_{0}^{(m)}\left(\mathbf{z}^{(m)}, \mathbf{z}_{1}^{(m)} ; \mathbf{z}^{\tau(m)}, \mathbf{z}_{1}^{\tau(m)}\right), \\
\ddot{\mathbf{b}}^{(m)}= & -2 \frac{\Omega}{m} \mathbf{k}_{1} \dot{\mathbf{c}}^{(m)}+\frac{\Omega^{2}}{m^{2}} \mathbf{k}_{2} \mathbf{b}^{(m)} \\
& +\mathbf{F}_{1}^{(m)}\left(\mathbf{z}^{(m)}, \mathbf{z}_{1}^{(m)} ; \mathbf{z}^{\tau(m)}, \mathbf{z}_{1}^{\tau(m)}\right), \\
\ddot{\mathbf{c}}^{(m)}= & 2 \frac{\Omega}{m} \mathbf{k}_{1} \dot{\mathbf{b}}^{(m)}+\frac{\Omega^{2}}{m^{2}} \mathbf{k}_{2} \mathbf{c}^{(m)} \\
& +\mathbf{F}_{2}^{(m)}\left(\mathbf{z}^{(m)}, \mathbf{z}_{1}^{(m)} ; \mathbf{z}^{\tau(m)}, \mathbf{z}_{1}^{\tau(m)}\right)
\end{aligned}
$$

where for $N=1,2, \ldots, \infty$.

$$
\begin{aligned}
& \mathbf{k}_{1}=\operatorname{diag}\left(\mathbf{I}_{n \times n}, 2 \mathbf{I}_{n \times n}, \ldots, N \mathbf{I}_{n \times n}\right), \\
& \mathbf{k}_{2}=\operatorname{diag}\left(\mathbf{I}_{n \times n}, 2^{2} \mathbf{I}_{n \times n}, \ldots, N^{2} \mathbf{I}_{n \times n}\right) ; \\
& \mathbf{b}^{(m)}=\left(\mathbf{b}_{1 / m}, \mathbf{b}_{2 / m}, \ldots, \mathbf{b}_{N / m}\right)^{\mathrm{T}}, \\
& \mathbf{c}^{(m)}=\left(\mathbf{c}_{1 / m}, \mathbf{c}_{2 / m}, \ldots, \mathbf{c}_{N / m}\right)^{\mathrm{T}} ; \\
& \mathbf{b}^{\tau(m)}=\left(\mathbf{b}_{1 / m}^{\tau}, \mathbf{b}_{2 / m}^{\tau}, \ldots, \mathbf{b}_{N / m}^{\tau}\right)^{\mathrm{T}}, \\
& \mathbf{c}^{\tau(m)}=\left(\mathbf{c}_{1 / m}^{\tau}, \mathbf{c}_{2 / m}^{\tau}, \ldots, \mathbf{c}_{N / m}^{\tau}\right)^{\mathrm{T}} ; \\
& \mathbf{F}_{1}^{(m)}=\left(\mathbf{F}_{11}^{(m)}, \mathbf{F}_{12}^{(m)}, \ldots, \mathbf{F}_{1 N}^{(m)}\right)^{\mathrm{T}}, \\
& \mathbf{F}_{2}^{(m)}=\left(\mathbf{F}_{21}^{(m)}, \mathbf{F}_{22}^{(m)}, \ldots, \mathbf{F}_{2 N}^{(m)}\right)^{\mathrm{T}} ; \\
& \mathbf{z}^{(m)}=\left(\mathbf{a}_{0}^{(m)}, \mathbf{b}^{(m)}, \mathbf{c}^{(m)}\right)^{\mathrm{T}}, \dot{\mathbf{z}}^{(m)}=\mathbf{z}_{1}^{(m)} ; \\
& \mathbf{z}^{\tau(m)}=\left(\mathbf{a}_{0}^{\tau(m)}, \mathbf{b}^{\tau(m)}, \mathbf{c}^{\tau(m)}\right)^{\mathrm{T}}, \dot{\mathbf{z}}^{\tau(m)}=\mathbf{z}_{1}^{\tau(m)}
\end{aligned}
$$

and for $k=1,2, \ldots, N$

$$
\begin{aligned}
& \mathbf{F}_{0}^{(m)}\left(\mathbf{z}^{(m)}, \mathbf{z}_{1}^{(m)} ; \mathbf{z}^{\tau(m)}, \mathbf{z}_{1}^{\tau(m)}\right) \\
& =\frac{1}{m T} \int_{0}^{m T} \mathbf{F}\left(\mathbf{x}^{(m) *}, \dot{\mathbf{x}}^{(m) *} ; \mathbf{x}^{\tau(m) *}, \dot{\mathbf{x}}^{\tau(m) *}, t, \mathbf{p}\right) d t ; \\
& \mathbf{F}_{1 k}^{(m)}\left(\mathbf{z}^{(m)}, \mathbf{z}_{1}^{(m)} ; \mathbf{z}^{\tau(m)}, \mathbf{z}_{1}^{\tau(m)}\right) \\
& =\frac{2}{m T} \int_{0}^{m T} \mathbf{F}\left(\mathbf{x}^{(m) *}, \dot{\mathbf{x}}^{(m) *} ; \mathbf{x}^{\tau(m) *}, \dot{\mathbf{x}}^{\tau(m) *}, t, \mathbf{p}\right) \cos \left(\frac{k}{m} \Omega t\right) d t, \\
& \mathbf{F}_{2 k}^{(m)}\left(\mathbf{z}^{(m)}, \mathbf{z}_{1}^{(m)} ; \mathbf{z}^{\tau(m)}, \mathbf{z}_{1}^{\tau(m)}\right) \\
& =\frac{2}{m T} \int_{0}^{m T} \mathbf{F}\left(\mathbf{x}^{(m) *}, \dot{\mathbf{x}}^{(m) *} ; \mathbf{x}^{\tau(m) *}, \dot{\mathbf{x}}^{\tau(m) *}, t, \mathbf{p}\right) \sin \left(\frac{k}{m} \Omega t\right) d t .
\end{aligned}
$$

The state-space form of Eq. (210) is

$\dot{\mathbf{z}}^{(m)}=\mathbf{z}_{1}^{(m)}$ and

$\dot{\mathbf{z}}_{1}^{(m)}=\mathbf{g}^{(m)}\left(\mathbf{z}^{(m)}, \mathbf{z}_{1}^{(m)} ; \mathbf{z}^{\tau(m)}, \mathbf{z}_{1}^{\tau(m)}\right)$

where

$$
\begin{gathered}
\mathbf{g}^{(m)}=\left(\mathbf{F}_{0}^{(m)},-2 \frac{\Omega}{m} \mathbf{k}_{1} \dot{\mathbf{c}}^{(m)}+\frac{\Omega^{2}}{m^{2}} \mathbf{k}_{2} \mathbf{b}^{(m)}+\mathbf{F}_{1}^{(m)},\right. \\
\left.2 \frac{\Omega}{m} \mathbf{k}_{1} \dot{\mathbf{b}}^{(m)}+\frac{\Omega^{2}}{m^{2}} \mathbf{k}_{2} \mathbf{c}^{(m)}+\mathbf{F}_{2}^{(m)}\right)^{\mathrm{T}} .
\end{gathered}
$$

An equivalent system of Eq. (213) is

$\dot{\mathbf{y}}^{(m)}=\mathbf{f}^{(m)}\left(\mathbf{y}^{(m)}, \mathbf{y}^{\tau(m)}\right)$

where

$$
\begin{gathered}
\mathbf{y}^{(m)}=\left(\mathbf{z}^{(m)}, \mathbf{z}_{1}^{(m)}\right)^{\mathrm{T}}, \mathbf{y}^{\tau(m)}=\left(\mathbf{z}^{\tau(m)}, \mathbf{z}_{1}^{\tau(m)}\right)^{\mathrm{T}} \\
\text { and } \mathbf{f}^{(m)}=\left(\mathbf{z}_{1}^{(m)}, \mathbf{g}^{(m)}\right)^{\mathrm{T}} .
\end{gathered}
$$

If equilibrium $\mathbf{y}^{(m) *}=\mathbf{y}^{\tau(m) *}$ of Eq. (215) (i.e., $\mathbf{f}\left(\mathbf{y}^{(m) *}, \mathbf{y}^{\tau(m) *}\right)$ $=\mathbf{0})$ exists, then the approximate analytical solution of period-m flow exists in Eq. (208). In vicinity of equilibrium $\mathbf{y}^{(m) *}=\mathbf{y}^{\tau(m) *}$, with $\mathbf{y}^{(m)}=\mathbf{y}^{(m) *}+\Delta \mathbf{y}^{(m)}$ and $\mathbf{y}^{\tau(m)}=$ $\mathbf{y}^{\tau(m) *}+\Delta \mathbf{y}^{\tau(m)}$, the linearized equation of Eq. (215) is

$$
\begin{aligned}
\Delta \dot{\mathbf{y}}^{(m)}= & D_{\mathbf{y}^{(m)}} \mathbf{f}^{(m)}\left(\mathbf{y}^{(m) *}, \mathbf{y}^{\tau(m) *}\right) \Delta \mathbf{y}^{(m)} \\
& +D_{\mathbf{y}^{\tau(m)}} \mathbf{f}^{(m)}\left(\mathbf{y}^{(m) *}, \mathbf{y}^{\tau(m) *}\right) \Delta \mathbf{y}^{\tau(m)}
\end{aligned}
$$

and the eigenvalue analysis of equilibrium $\mathbf{y}^{(m) *}=\mathbf{y}^{\tau(m) *}$ is given by

$$
\begin{aligned}
& \mid D_{\mathbf{y}^{(m)}} \mathbf{f}^{(m)}\left(\mathbf{y}^{(m) *}, \mathbf{y}^{\tau(m) *}\right)-\lambda \mathbf{I}_{2 n(2 N+1) \times 2 n(2 N+1)} \\
& \quad+D_{\mathbf{y}^{\tau(m)}} \mathbf{f}^{(m)}\left(\mathbf{y}^{(m) *}, \mathbf{y}^{\tau(m) *}\right) e^{-\lambda \tau} \mid=0
\end{aligned}
$$

where

$$
\begin{aligned}
& D_{\mathbf{y}^{(m)}} \mathbf{f}^{(m)}\left(\mathbf{y}^{(m) *}, \mathbf{y}^{\tau(m) *}\right) \\
& =\partial \mathbf{f}^{(m)}\left(\mathbf{y}^{(m)}, \mathbf{y}^{\tau(m)}\right) /\left.\partial \mathbf{y}^{(m)}\right|_{\left(\mathbf{y}^{(m) *}, \mathbf{y}^{\tau(m) *}\right)}, \\
& D_{\mathbf{y}^{\tau(m)}} \mathbf{f}^{(m)}\left(\mathbf{y}^{(m) *}, \mathbf{y}^{\tau(m) *}\right) \\
& =\partial \mathbf{f}^{(m)}\left(\mathbf{y}^{(m)}, \mathbf{y}^{\tau(m)}\right) /\left.\partial \mathbf{y}^{\tau(m)}\right|_{\left(\mathbf{y}^{(m) *}, \mathbf{y}^{\tau(m) *}\right)} .
\end{aligned}
$$


Thus, the stability and bifurcation of periodic solution can be classified by the eigenvalues of Eq. (217) at equilibrium $\mathbf{y}^{(m) *}=\mathbf{y}^{\tau(m) *}$ with

$\left(n_{1}, n_{2}, n_{3} \mid n_{4}, n_{5}, n_{6}\right)$

(i) If all eigenvalues of the equilibrium possess negative real parts, the approximate periodic solution is stable.

(ii) If at least one of eigenvalues of the equilibrium possesses positive real part, the approximate numerical solution is unstable.

(iii) The boundaries between stable and unstable equilibriums with higher order singularity give bifurcation and stability conditions with higher order singularity.

Proof The proof is similar to the proof of Theorem 14.

\section{Conclusion}

From Luo [39], the analytical dynamics of periodic flows and chaos in nonlinear dynamical systems were reviewed. The analytical solutions of periodic flows and chaos in autonomous systems were presented first, and the analytical dynamics of periodically forced, nonlinear dynamical systems was presented. The analytical solutions of periodic motions in free and periodically forced, nonlinear vibration systems were also presented. Extended was the above ideas to the time-delayed, nonlinear dynamical systems, and the analytical solutions of periodic flows for time-delayed, nonlinear systems were investigated with/without periodic excitations, and time-delayed vibration systems were also discussed due to extensive applications. The analytical solutions of periodic flows and chaos are independent of the small parameters, which are different from the traditional perturbation methods. The methodology presented herein will provide accurate analytical solutions of periodic motions to chaos in nonlinear dynamical systems with/without time-delay. This approach can handle nonlinear dynamical systems with either single time-delay or multiple time-delays. Such analytical method was applied to sampled problems relative to dynamical systems without time-delay already, which can be found from Luo and Huang [40-47] and Luo and Yu [48-50]. Examples for analytical solutions of periodic solutions in time-delayed nonlinear systems will be presented in sequel.

\section{References}

1. Lagrange JL (1788) Mecanique analytique, vol 2. Albert Balnchard, Paris

2. Pioncare H(1892) Les methods nouvelles de la mechanique celeste. Gauthier-Villars, Paris
3. Birkhoff GD (1913) Proof of Poincare's geometric theorem. Trans Am Math Soc 14:14-22

4. Birkhoff GD (1927) Dynamical systems. American Mathematical Society, New York

5. van der Pol B (1920) A theory of the amplitude of free and forced triode vibrations. Radio Rev 1:701-710, 754-762

6. van der Pol B, van der Mark J (1927) Frequency demultiplication. Nature 120:363-364

7. Cartwright ML, Littlewood JE (1945) On nonlinear differential equations of the second order I. The equation $\ddot{y}-k\left(1-y^{2}\right) \dot{y}+y=$ $b \lambda k \cos (\lambda t+\alpha), k$ large. J Lond Math Soc 20:180-189

8. Cartwright ML, Littlewood JE (1947) On nonlinear differential equations of the second order II. The equation $\ddot{y}+k f(y) \dot{y}+$ $g(y, k)=p(t)=p_{1}(t)+k p_{2}(t), k>0, f(y) \geq 1 .$. Ann Math 48:472-494

9. Levinson N (1948) A simple second order differential equation with singular motions. Proc Natl Acad Sci USA 34(1):13-15

10. Levinson N (1949) A second order differential equation with singular solutions. Ann Math (second series) 50(1):127-153

11. Smale S (1967) Differentiable dynamical systems. Bull Am Math Soc 73:747-817

12. Melnikov VK (1962) On the behavior of trajectories of system near to autonomous Hamiltonian systems. Sov Math Dokl 3:109112

13. Melnikov VK (1963) On the stability of the center for time periodic perturbations. Trans Mosc Math Soc 12:1-57

14. Duffing G (1918) Erzwunge Schweingungen bei veranderlicher eigenfrequenz. F. Viewig u. Sohn, Braunschweig

15. Fatou P (1928) Sure'le mouvement d'un systeme soumis a' des forces a courte periode. Bull Soc Math 56:98-139

16. Krylov NM, Bogolyubov NN (1935) Methodes approchees de la mecanique non-lineaire dans leurs application a l'Aeetude de la perturbation des mouvements periodiques de divers phenomenes de resonance s'y rapportant. Academie des Sciences d'Ukraine, Kiev

17. Bogoliubov N, Mitropolsky Y (1961) Asymptotic methods in the theory of nonlinear oscillations. Gordon and Breach, New York

18. Stoker JJ (1950) Nonlinear vibrations. Wiley, New York

19. Minorsky N (1962) Nonlinear oscillations. Van Nostrand, New York

20. Hayashi C (1964) Nonlinear oscillations in physical systems. McGraw-Hill, New York

21. Nayfeh AH (1973) Perturbation methods. Wiley, New York

22. Holmes PJ, Rand DA (1976) Bifurcations of Duffing equation: an application of catastrophe theory. Q Appl Math 35:495-509

23. Nayfeh AH, Mook DT (1979) Nonlinear oscillation. Wiley, New York

24. Holmes PJ (1979) A nonlinear oscillator with strange attractor. Philos Trans R Soc A 292:419-448

25. Ueda Y (1980) Explosion of strange attractors exhibited by the Duffing equations. Annu N Y Acad Sci 357:422-434

26. Greenspan BD (1981) Bifurcations in periodically forced oscillations: subharmonics and homoclinic orbits. Ph.D. thesis, Center for Applied Mathematics, Cornell University, Ithaca

27. Greenspan BD, Holmes PJ (1983) Homoclinic orbits, subharmonics and global bifurcations in forced oscillations. In: Barenblatt G, Iooss G, Joseph DD (eds) Nonlinear dynamics and turbulence. Pitman, London

28. Guckenheimer J, Holmes P (1983) Nonlinear oscillations, dynamical systems, and bifurcations of vector fields. Springer, New York

29. Luo ACJ (1995) Analytical modeling of bifurcations, chaos and multifractals in nonlinear dynamics. Ph.D. Dissertation, University of Manitoba, Winnipeg

30. Luo ACJ, Han RPS (1999) Analytical predictions of chaos in a nonlinear rod. J Sound Vib 227(3):523-544 
31. Arnold VI (1964) Instability of dynamical systems with several degrees of freedom. Sov Math Dokl 5:581-585

32. Chirikov BV (1979) A universal instability of many-dimensional oscillator systems. Phys Rep 52:263-379

33. Wang CS, Kao YH, Huang JC, Gou YH (1992) Potential dependence of the bifurcation structure in generalized Duffing oscillators. Phys Rev A 45:3471-3485

34. Coppola VT, Rand RH (1990) Averaging using elliptic functions: approximation of limit cycle. Acta Mech 81:25-142

35. Luo ACJ, Han RPS (2001) The resonance theory for stochastic layers in nonlinear dynamical systems. Chaos Solitons Fractals 12:2493-2508

36. Kao YH, Wang CS, Yang TH (1992) Influences of harmonic coupling on bifurcations in Duffing oscillator with bounded potential wells. J Sound Vib 159:13-21

37. Luo ACJ, Han RPS (1997) A quantitative stability and bifurcation analyses of a generalized Duffing oscillator with strong nonlinearity. J Frankl Inst 334B:447-459

38. Peng ZK, Lang ZQ, Billings SA, Tomlinson GR (2008) Comparisons between harmonic balance and nonlinear output frequency response function in nonlinear system analysis. J Sound Vib 311:56-73

39. Luo ACJ (2012) Continuous dynamical systems. Higher Education Press/L\&H Scientific, Beijing/Glen Carbon

40. Luo ACJ, Huang J (2012) Approximate solutions of periodic motions in nonlinear systems via a generalized harmonic balance. J Vib Control 18:1661-1671

41. Luo ACJ, Huang J (2012) Analytical dynamics of period-m flows and chaos in nonlinear systems. Int J Bifurc Chaos 22(4):29

42. Luo ACJ, Huang JZ (2012) Analytical routes of period-1 motions to chaos in a periodically forced Duffing oscillator with a twin-well potential. J Appl Nonlinear Dyn 1:73-108

43. Luo ACJ, Huang JZ (2012) Unstable and stable period- $m$ motions in a twin-well potential Duffing oscillator. Discontin Nonlinearity Complex 1:113-145

44. Luo ACJ, Huang JZ (2013) Analytical solutions for asymmetric periodic motions to chaos in a hardening Duffing oscillator. Nonlinear Dyn 72:417-438

45. Luo ACJ, Huang JZ (2013) Analytical period-3 motions to chaos in a hardening Duffing oscillator. Nonlinear Dyn 73:1905-1932

46. Luo ACJ, Huang JZ (2013) An analytical prediction of period1 motions to chaos in a softening Duffing oscillator. Int J Bifurc Chaos 23:Article No: 1350086 (31 pages)
47. Luo ACJ, Huang JZ (2013) Period-3 motions to chaos in a softening Duffing oscillator. Int J Bifurc Chaos (in press)

48. Luo ACJ, Yu B (2013) Analytical solutions for stable and unstable period-1 motion in a periodically forced oscillator with quadratic nonlinearity. ASME J Vib Acoust 135:Article No: 034503 (5 pages)

49. Luo ACJ, Yu B(2013) Complex period-1 motions in a periodically forced, quadratic nonlinear oscillator. J Vib Control. doi:10.1177/ 1077546313490525

50. Luo ACJ, Yu B (2013) Period- $m$ motions and bifurcation trees in a periodically excited, quadratic nonlinear oscillator. Discontin Nonlinearity Complex (in press)

51. Luo ACJ, Laken AB (2013) Analytical solutions for period-m motions in a periodically forced van der Pol oscillator. Int J Dyn Control 1(2):99-115

52. Tlusty J (2000) Manufacturing processes and equipment. Prentice Hall, Upper Saddle River

53. Hu HY, Wang ZH (2002) Dynmaics of controlled mechanical systems with delayed feedback. Springer, Berlin

54. Stepan G (1989) Retarded dynamical systems. Longman, Harlow

55. Sun JQ (2009) A method of continuous time approximation of delayed dynamical systems. Commun Nonlinear Sci Numer Simul 14(4):998-1007

56. Insperger T, Stepan G (2011) Semi-discretization for time-delay systems: stability and engineering applications. Springer, New York

57. Hu HY, Dowell EH, Virgin LN (1998) Resonance of harmonically forced Duffing oscillator with time-delay state feedback. Nonlinear Dyn 15(4):311-327

58. Wang H, Hu HY (2006) Remarks on the perturbation methods in solving the second order delay differential equations. Nonlinear Dyn 33:379-398

59. MacDonald N (1995) Harmonic balance in delay-differential equations. J Sounds Vib 186(4):649-656

60. Liu L, Kalmar-Nagy T (2010) High-dimensional harmonic balance analysis for second-order delay-differential equations. J Vib Control 16(7-8):1189-1208

61. Leung AYT, Guo Z (2012) Bifurcation of the periodic motions in nonlinear delayed oscillators. J Vib Control. doi:10.1177/ 1077546312464988 